\title{
Probleme im Forschungsstand über phänomenales Erleben
}

In diesem Kapitel konzentriere ich mich auf zwei Aspekte zum Forschungsstand in der Philosophie des Geistes, die für diese Arbeit bzw. im Hinblick auf phänomenales Erleben von zentraler Bedeutung sind: 1.) die drei Argumenttypen der Qualia-Befürworter/innen; 2.) Probleme, die sich im Umgang mit dem hard problem und dessen Explanandum ausmachen lassen.

\subsection{Drei Argumenttypen von Reduktionismus-SkeptikerInnen}

Nach Walter bestehen die drei wesentlichen Argumenttypen von Reduktionismus-SkeptikerInnen ${ }^{1}$ bzw. »Qualia-Freunden $\aleph^{2}$ in epistemischen, modalen und auf der Erklärungslücke basierenden explanatorischen Argumenten. ${ }^{3}$ In den folgenden Abschnitten werde ich darlegen, warum sich im Hinblick auf alle drei Argumenttypen für reduktionsoptimistische Positionen im besten Fall eine Patt-Situation aufrechterhalten lässt.

1 Unter >Reduktionismus-SkeptikerInnen $<$ - oder einfacher >Reduktions-SkeptikerInnen $<$ verstehe ich Vertreter von Positionen in der Philosophie des Geistes, die der Behauptung, dass Phänomenales vollständig bzw. hinreichend auf nicht-phänomenale Eigenschaften reduziert (und mit letzteren erklärt) werden können, skeptisch gegenüber stehen. (Dazu gehört bspw. auch die Skepsis, dass mit Dritte-Person-Eigenschaften, Erste-PersonEigenschaften epistemisch erfasst werden können.)

2 Walter, Sven: »Allgemeine Einleitung: Phänomenales Bewusstsein: Unlösbares Mysterium oder seriöses wissenschaftliches Problem?«, in: Qualia. Ausgewählte Beiträge, Heckmann, Heinz-Dieter / Walter, Sven [Hrsg.], Paderborn: Mentis 2001, S. 24.

3 Auch die in Chalmers Conscious Mind aufgeführten fünf Argumente gegen die physikalistische Reduzierbarkeit von bewusstem Erleben lassen sich diesen drei Argumenttypen zuordnen. Vgl. Chalmers, David J.: The conscious mind. In search of a fundamental theory, New York: Oxford University Press 1996, S. 94-106. Im Hinblick auf den modalen Argumenttyp spricht er bspw. von »arguments from conceivability« und bei einem Argument das dem explanatorischen Typ zugewiesen werden kann, von einem »argument from analysis«. A.a.O., S. 94 . 


\subsubsection{Die explanatorische Lücke: Kontingente Korrelate von Erleben}

[...] unser Naturerkennen gelangt an eine Kluft, über die kein Steg, kein Fittich trägt: [...] Dies Unbegreifliche ist das Bewusstsein. [...] Dass nicht allein bei dem heutigen Stand unserer Kenntnis das Bewusstsein aus seinen materiellen Bedingungen nicht erklärbar ist, was wohl jeder zugibt, sondern dass es auch der Natur der Dinge nach aus diesen Bedingungen nicht erklärbar sein wird. ${ }^{4}$

Die hier zitierte, von Du Bois-Reymonds 1872 thematisierte Kluft der Erklärbarkeit wurde 1983 von Joseph Levine mit dem Begriff der »Erklärungslücke« (»explanatory gap «) $)^{5}$ systematisch relevant und im Kontext der Diskussion um Kripke's Argument ${ }^{6}$ gegen die Gehirn-Geist-Identität eingeführt. Die Erklärungslücke hatte von Anfange an eine wichtige Rolle im Diskurs über phänomenales Bewusstsein. ${ }^{7}$ Analog zum Konzept des hard problem ${ }^{8}$ von Chalmers oder zu Nagels Überlegungen »What is it like to be a bat? ${ }^{9}{ }^{\text {drückt }}$ die explanatorische Lücke nach Levine ein fundamentales Rätsel aus: »[...] the idea that there exists an explanatory gap expresses the deep puzzle concerning the place of conscious experience in a material world $\ll .{ }^{10}$

Das Argument der Erklärungslücke weist auf ein explanatorisches Defizit unseres physikalischen bzw. neurophysiologischen Wissens hin, insofern die Neurowissenschaften den Zusammenhang zwischen bestimmten neurophysiologischen Zuständen und dem Auftreten bestimmter phänomenaler

Du Bois-Reymond, Emil: Über die Grenzen des Naturerkennens. Vorträge über Philosophie und Gesellschaft, Hamburg: Meiner [zuerst 1872]/1974, S. 65.

5 Levine, Joseph: »Materialism and Qualia: The Explanatory Gap«, Pacific Philosophical Quarterly, [1983] Vol. 64, No. 4, S. 357.

6 Vgl. Kripke, Saul A.: Naming and necessity, Oxford: Blackwell [zuerst 1972]/1980.

7 Vgl. Schildknecht, Christiane: Sense and self. Perspectives on nonpropositionality, Paderborn: Mentis 2002, S. 241.

8 Vgl. Chalmers, David J.: The conscious mind. In search of a fundamental theory, New York: Oxford University Press 1996, S. 25.

9 Nagel, Thomas: »What is it like to be a bat?«, Philosophical Review, [1974] Vol. 83, No. 4. In Kombination mit Nagels Überlegungen lässt sich das Argument der Erklärungslücke folgendermassen formulieren: »A neural oscillation of so and so many Hertz does not explain what it is like to be us«. Schildknecht, Christiane: Sense and self. Perspectives on nonpropositionality, Paderborn: Mentis 2002, S. 241.

10 Levine, Joseph: »The Explanatory Gap«, in: The Oxford Handbook of Philosophy of Mind, McLaughlin, Brian P. / Beckermann, Ansgar / Walter, Sven [Hrsg.], Oxford: Oxford University Press 2009, S. 281. Im Hinblick auf das Phänomen bewussten Erlebens, können wir Chalmers zufolge mit dem explanatorischen Argument die reduktionskritische Diagnose erstellen, dass es keine Konzeption von Erleben gibt, die begründet, warum es mit physiologischen Vorgängen identisch sein soll. Vgl. Chalmers, David J.: The conscious mind. In search of a fundamental theory, New York: Oxford University Press 1996, S. 94. 
Erlebnisse nicht erklären bzw. bestimmte neurophysiologische Konstrukte nicht als Erklärungen für das Auftreten phänomenaler Erlebnisse ${ }^{11}$ ausweisen können: »[...] what is left unexplained by the discovering of C-fiber firing is why pain should feel the way it does! [...] Unlike its functional role, the identification of the qualitative side of pain with C-fiber firing [...] leaves the connection between it and what we identify it with completely mysterious « ${ }^{12}$ Das Erklärungslücken-Argument Levines bezieht sich also auf die fehlende Erklärungskraft, die neuronale Korrelate, im Hinblick auf den Aspekt von phänomenalem Erleben haben. Dieser Mangel an explanatorischer Kraft besteht, wenn wir auch nur einen einigermassen anspruchsvollen Erklärungsbegriff voraussetzen.

Um dem explanatorischen Argument beizukommen, wird daher zuweilen versucht, derart wenige Ansprüche an den Erklärungsbegriff zu stellen, dass eine Korrelation von neurophysiologischen Ereignissen und subjektiven Erlebnisberichten bereits als Erklärung des subjektiven Erlebens oder sogar als $>$ Erklärung`für ihre angebliche >Identität‘ gilt. ${ }^{13}$ Eine Variante dieser Strategie

11 Was soll eigentlich genau erklärt sein, wenn wir bspw. feststellen, dass Schmerz das >Feuern von C-Fasern sei? C-Fasern mögen zwar eine wichtige Rolle bei Schmerzempfindungen spielen. Unsere Konzeption über das, was wir >Schmerzen jedoch auch, ja im Wesentlichen den Aspekt des Erlebens von Schmerz, der bei einem kausalen oder funktionalen Ansatz unerklärt bleibt. Vgl. dazu auch die Kritik von Levine, Joseph: »Materialism and Qualia: The Explanatory Gap«, Pacific Philosophical Quarterly, [1983] Vol. 64, No. 4, S. 357 .

12 Ebd.

13 So haben bspw. Block und Stalnaker Levines Modell einer reduktiven Erklärung als unangemessen zurückgewiesen und es durch ein anderes, weniger anspruchsvolles Modell ersetzt. Vgl. dazu Block, Ned J. / Stalnaker, Robert: »Begriffsanalyse, Dualismus und die Erklärungslücke«, in: Begriff, Erklärung, Bewusstsein. Neue Beiträge zum Qualia-Problem, Pauen, Michael / Schütte, Michael / Staudacher, Alexander [Hrsg.], Paderborn: Mentis [zuerst 1999, engl.]/2007, S. 61-110. Bei diesem Modell werden die (eigentlich zu beweisenden) theoretischen Identitätsaussagen vorausgesetzt. Die Rechtfertigung dafür ist ein Rückgriff auf die (eigentlich in Frage stehende) explanatorische Kraft theoretischer Identitätsaussagen. Mit den zwei Voraussetzung von a.) theoretischen Identität und b.) der angeblichen »explanatorischen Leistungskraft « theoretischer Identitätsaussagen wird also versucht, die explanatorische Kraft einer reduktiven Erklärung zu erhöhen: »[...] Diesem neuen Modell zufolge ergeben sich die zu erklärenden theoretischen Identitätsaussagen nicht als deduktive Folgerungen aus einer a-priori-Analyse einerseits und empirischer Information in Begriffen der reduzierenden Beschreibungsebene andererseits. Vielmehr werden in diesem neuen Modell die reduktiv zu erklärenden Identitätsaussagen begründet, indem ihre explanatorische Leistungskraft herausgestellt wird. D.h. die theoretischen Identitätsaussagen werden gerechtfertigt, indem nachgewiesen wird, dass sich unter Voraussetzung ihrer Geltung die charakteristischen Eigenschaften des zu reduzierenden Phänomenbereichs erklären lassen. Ihre Rechtfertigung erhalten theoretische Identitätsaussagen also durch ihre explanatorische Leistungskraft«. Thöle, 
bestünde darin, zu behaupten, die explanatorische Lücke sei bloss eine Folge von begrifflichem Konservativismus (im Hinblick auf den Erklärungsbegriff). ${ }^{14}$

Die für die Erklärungslücke verantwortliche Kontingenz (bzw. ein »sense of arbitrariness «), ${ }^{15}$ die wir zwischen neuronalem Korrelat und Erlebnis feststellen können, ist zweifältig: einerseits können wir fragen, warum überhaupt mit einem neuronalen Muster ein Erlebnis einhergeht, andererseits kann man fragen, warum dieser neuronaler Vorgang genau von jenem bestimmten Erleben und nicht von einem anderen begleitet wird:

\begin{abstract}
Warum veranlasst dieses Muster neuraler Aktivitäten einen Gelbeindruck und nicht einen Blaueindruck oder den Eindruck von etwas Bitterem oder einen ganz anderen Sinneseindruck? Warum tritt überhaupt mit diesem Muster neuraler Aktivitäten auch ein Sinneseindruck auf? Was an den physikalischchemischen Vorgängen hilft, die qualitative Ausprägung sinnlicher Qualitäten im sensorischen Bewusstsein [sic!] [zu] erklären?16
\end{abstract}

Chalmers verortet grundsätzlich vier verschiedene Reaktionen in der Diskussion um die Erklärungslücke: 1. evasiv und ignorierend: »Some deny that any explanatory gap exists at all «;17 2. das Hoffen auf spätere Fortschritte der Naturwissenschaften: »Some hold that there is an explanatory gap for now, but that it will eventually be closed «; $; i^{18} 3$. Dualismus oder Eigenschaftsdualismus: »Some hold that the explanatory gap corresponds to an ontological gap

Bernhard: »Bewusstsein und Erklärungslücken«, in: Begriff, Erklärung, Bewusstsein. Neue Beiträge zum Qualia-Problem, Pauen, Michael / Schütte, Michael / Staudacher, Alexander [Hrsg.], Paderborn: Mentis 2007, S. 226 [Hervorhebung im Original].

Doch selbst wenn wir die Behauptung akzeptieren würden, dass das Feuern von bestimmten Neuronen phänomenales Erleben >erklärt<, sobald wir von einem deflationären Erklärungsbegriff ausgehen, fehlt auf der Ebene dieser reduktiven `Erklärung < (Neuronenfeuer) das zu rettende Phänomen des bewussten Erlebens. Das bedeutet, dass das Prinzip der Rettung der Phänomene auch mit einer Bedeutungsverschiebung des Erklärungsbegriffs nicht gewährleistet ist. Eine ähnliche Strategie findet sich bei Papineau. Vgl. dazu Papineau, David: »Mind the Gap«, Philosophical Perspectives, [1998] Vol. 12: Language, Mind, and Ontology, S. 384.

14 Vgl. dazu auch Staudacher, Alexander: Phänomenales Bewusstsein als Problem für den Materialismus, Berlin: De Gruyter 2002, S. 371-372.

15 Levine, Joseph: »Phenomenal concepts and the materialist constraint «, in: Phenomenal concepts and phenomenal knowledge. New essays on consciousness and physicalism, Alter, Torin / Walter, Sven [Hrsg.], Oxford: Oxford University Press 2007, S. 145.

16 Lanz, Peter:Das phänomenale Bewusstsein. Eine Verteidigung, Frankfurt am Main: Klostermann 1996, S. 131 [Hervorhebung E. E.; Ergänzung in Klammern von E.E.].

17 Chalmers, David J.: »Phenomenal concepts and the explanatory gap«, in: Phenomenal concepts and phenomenal knowledge. New essays on consciousness and physicalism, Alter, Torin / Walter, Sven [Hrsg.], Oxford: Oxford University Press 2007, S. 167.

Ebd. 
in nature « $; i^{19}$ 4. Die Konzeption phänomenaler Begriffe: »Those who [...] agree that there is an explanatory gap [...] hold that it stems from the way we think about consciousness « ${ }^{20}$

Der von Chalmers kritisierte Versuch, explanatorische Argumente im Rückgriff auf die Hoffnung zukünftiger naturwissenschaftlicher Fortschritte (bspw. der Neuro- oder Kognitionswissenschaften) zu entkräften, besteht zumeist im Hinweis auf vermeintliche ${ }^{21}$ Analogien zu anderen physikalischen Reduktionen $^{22}$ aus der Wissenschaftsgeschichte. Die aufgeführten Analogien beziehen sich immer auf Fälle, in denen Makroeigenschaften problemlos mit Mikroeigenschaften erklärt werden können. Beispielsweise können die (Makro-) Eigenschaften von Wasser schlüssig durch die Mikro-Eigenschaft von $\mathrm{H}^{2} \mathrm{O}$-Molekülen erklärt werden. Eine Erklärung von phänomenalen (Makro-) Eigenschaften wie Farberlebnissen durch neuronale Eigenschaften, bspw. von Vorgängen in der Sehrinde, ist jedoch alles andere als befriedigend und wirkt, weil sich auf der Mikroebene keine analogen Eigenschaften ${ }^{23}$ finden lassen, leider kontingent. ${ }^{24}$ Die Erklärungslücke von Levine zeigt sich vielmehr als einzigartiges Problem intertheoretischer Reduktion:

\section{Ebd.}

20 Ebd. Die Lücke wird demgemäss nur zwischen unseren Begriffen über physikalische Prozesse und unseren Begriffen über Bewusstsein gesehen. Die Konzeption der >phänomenalen Begriffe wird auch als Einwand gegen epistemische Argumente angewendet. Vgl. weiter unten.

21 In Abschnitt 6.4 gehe ich näher darauf ein, warum es sich bei diesen Analogien nur um vermeintliche Analogien handelt.

22 So konstatiert Loar, dass wir das Urteil »[...] diese Substanz ist $\mathrm{CH}_{3} \mathrm{CH}_{2} \mathrm{OH}[\ldots]$ « nicht besorgniserregend fänden, obwohl dies ja auch nicht a priori feststehe. Loar, Brian: »Phänomenale Zustände«, in: Begriff, Erklärung, Bewusstsein. Neue Beiträge zum QualiaProblem, Pauen, Michael / Schütte, Michael / Staudacher, Alexander [Hrsg.], Paderborn: Mentis [zuerst 1997, engl.]/2007, S. 47. Die Erklärungslücke sei deshalb nur ein epistemisches oder begriffliches Problem und belege nur die Tatsache der begrifflichen Nichtäquivalenz von Typ-Demonstrativa des Wiedererkennens (d.h. Wiedererkennungsbegriffen) und deskriptiven Begriffen über physikalische Eigenschaften. Vgl. ebd.

23 Ein neuronaler Vorgang, der mit Farbwahrnehmung zu tun hat, weist keinerlei Farbeigenschaften auf: »[...] color turns out to occur only in experiences and nowhere else, all the other items in the story being only causes of conditions that, in the end, cause qualia «. Robinson, William S.: »Qualia realism«, in: A Field Guide to the Philosophy of Mind, SIFA, Società Italiana Filosofia Analitica, 2002, Online: http://host.uniromaz.it/progetti/kant/ field/qr.htm [zuletzt geprüft am 25.09.2018], Abschnitt »The Argument from Science«.

24 Vgl. Block, Ned J. / Stalnaker, Robert: »Begriffsanalyse, Dualismus und die Erklärungslücke«, in: Begriff, Erklärung, Bewusstsein. Neue Beiträge zum Qualia-Problem, Pauen, Michael / Schütte, Michael / Staudacher, Alexander [Hrsg.], Paderborn: Mentis [zuerst 1999, engl.]/2007, S. 64 . 
$[\ldots]$ it opens an >explanatory gap not encountered in other cases of intertheoretical [sic!] reduction, since the qualitative character of an experience cannot be deduced from any physical or functional description of it. Such attempts thus pose, at very least, a unique epistemological problem for functionalist (or physicalist) reductions of qualitative states. ${ }^{25}$

Infolgedessen ist das Aufzeigen von Korrelationen zwischen mentalen >Makroeigenschaften < und physikalischen Mikroeigenschaften - im Gegensatz zum Nachweis von Mikroeigenschaften, die Makroeigenschaften plausibel erklären - nur von deskriptivem und nicht von explanatorischem Wert:

In the fundamental sciences, one typically speaks of causal relations in terms of interactions. In physics, for instance, there are four fundamental kinds of interactions (electromagnetic, weak, strong, gravitational) which serve to explain the correlations that are observed in physical systems. As regards the mind-matter problem, the situation is more difficult. Far from a theoretical understanding in this field, the existing body of knowledge essentially consists of empirical correlations between material and mental states. These correlations are descriptive, not explanatory; they are not causally conditioned. ${ }^{26}$

Reduktionsskeptiker schliessen demgemäss aus der Erklärungslücke auf eine grundlegende epistemische und/oder auf eine ontologische Lücke. ${ }^{27}$

25 Levin, Janet: »Functionalism«, in: The Stanford Encyclopedia of Philosophy, 2013, Online: http://plato.stanford.edu/archives/fall2013/entries/functionalism/ [zuletzt geprüft am 28.o9.2018], Abschnitt 5.5.2.

26 Atmanspacher, Harald: »Quantum Approaches to Consciousness«, in: The Stanford Encyclopedia of Philosophy, 2015, Online: http://plato.stanford.edu/archives/sum2015/ entries/qt-consciousness [zuletzt geprüft am 28.o9.2018], Abschnitt 2.o.

27 So ist bspw. nach Jackson und Chalmers ein reduktiver Materialismus falsch, weil Phänomenales nicht identisch mit gängigen physikalischen Eigenschaften sein kann, was auf eine ontologische Lücke hinweist (z.B. Dualismus oder Eigenschaftsdualims). Nach Levine steht der Materialismus nur vor einem epistemischen Problem. Vgl. dazu Staudacher, Alexander: »Begriffsanalyse und Erklärungslücke«, in: Begriff, Erklärung, Bewusstsein. Neue Beiträge zum Qualia-Problem, Pauen, Michael / Schütte, Michael / Staudacher, Alexander [Hrsg.], Paderborn: Mentis 2007, S. 242. Eine gute Übersicht von verschiedenen Interpretationen welche epistemischen, wissenschaftstheoretischen und ontologischen Konsequenzen der explanatory gap haben könnte, bietet Tye, Michael: »Qualia«, in: The Stanford Encyclopedia of Philosophy, 2007, Online: http://plato.stanford. edu/archives/fall2007/entries/qualia/ [zuletzt geprüft am 25.09.2018], Abschnitt 5.o. Vgl. auch Van Gulick, Robert: »Consciousness«, in: The Stanford Encyclopedia of Philosophy, 2014, Online: http://plato.stanford.edu/archives/spr2014/entries/consciousness/ [zuletzt geprüft am 17.09.2018], Abschnitt 5.2. 
Sven Walter meint diesbezüglich, dass die $» I n t u i t i o n ~ «,{ }^{28}$ auf der das Erklärungslückenargument basiere, »irreführend $\aleph^{29}$ sein könnte und verweist auf $»$ Analogien $\ll^{30}$ aus den Erfolgen der Wissenschaftsgeschichte, auf die sich Physikalisten mindestens prima facie zurückziehen könnten. ${ }^{31}$ Folglich wird von ihm, zumindest an dieser Stelle, die Beweislast den Reduktions-Skeptikern zugeschoben. ${ }^{32}$ So stellt er fest: »Die Qualia-Freunde sind gezwungen, Argumente gegen den Physikalismus zu formulieren, die nicht mit dem zukünftigen Erfolg oder Misserfolg der Neuro- und Kognitionswissenschaften stehen und fallen $[\ldots] \ll 3^{33}$

Hier gilt es jedoch zu bedenken, dass das Erklärungslückenargument gerade ein Beispiel für die, in seinem Zitat verlangten »Argumente« darstellt. Denn es zielt darauf $a b$, dass die festgestellte Kontingenz zwischen neuronalem Korrelat und Erlebnis (bzw. die damit zusammenhängende Erklärungslücke) prinzipieller Natur ist. Allfällige, zukünftige Erfolge der Neurowissenschaften wären im Hinblick auf den Kern des Erklärungslückenarguments gar nicht relevant. Die Erklärungslücke wird nicht schmäler, wenn genaueres oder sogar salles` über diese Korrelate herausgefunden wird, denn damit würde, um Gerhard Roths Terminologie zu verwenden, nur die »Sprache der Neurone $«^{34}$ differenzierter und detaillierter erfasst. Mit anderen Worten: nicht die Lücke würde eingeebnet, sondern die >nicht-phänomenale Flankeく diesseits der Lücke würde genauer oder im besten Fall genauestens erfasst. ${ }^{35}$

28 Walter, Sven:»Allgemeine Einleitung: Phänomenales Bewusstsein:Unlösbares Mysterium oder seriöses wissenschaftliches Problem?«, in: Qualia. Ausgewählte Beiträge, Heckmann, Heinz-Dieter / Walter, Sven [Hrsg.], Paderborn: Mentis 2001, S. 28.

29 Ebd.

30 Ebd.

31 Vgl. ebd.

32 Auf diese Problematik gehe ich in Abschnitt 4.2.3 näher ein.

33 Ebd.

34 Roth, Gerhard: Das Gehirn und seine Wirklichkeit. Kognitive Neurobiologie und ihre philosophischen Konsequenzen, Frankfurt am Main: Suhrkamp 1994, S. 22.

35 Auf dieses prinzipielle Problem geht paradoxerweise auch Sven Walters Formulierung der Erklärungslücke ein: »Unabhängig davon, wie detailliert unser empirisches Wissen wird, verstehen wir nämlich nicht, warum gerade diese Prozesse von diesen Erlebnissen beleitet sein sollen oder warum sie überhaupt von irgendwelchen Erlebnissen begleitet sein sollten [...]«. Walter, Sven: »Allgemeine Einleitung: Phänomenales Bewusstsein: Unlösbares Mysterium oder seriöses wissenschaftliches Problem?«, in: Qualia. Ausgewählte Beiträge, Heckmann, Heinz-Dieter / Walter, Sven [Hrsg.], Paderborn: Mentis 20o1, S. 2627 [Hervorhebung im Original]. 
Das Argument der Erklärungslücke wird also gestützt, wenn sich ausweisen lässt, dass die vielzitierten »Analogien «36 aus der Wissenschaftsgeschichte nicht greifen, und dass infolgedessen auch »zukünftige Erfolge $\aleph^{37}$ der Neurowissenschaften, die Erklärungslücke nicht schliessen könnten (vgl. dazu Abschnitt 6.4). Das heisst aber nicht zwingend, dass bewusstes Erleben für immer etwas Unerklärliches bleiben muss, sondern nur, dass eine reduktive Erklärung im Sinne eines strikten, identitätstheoretischen Physikalismus in dem es »keinen Platz« ${ }^{38}$ für Erleben gibt, aufgegeben werden muss:

We certainly need not give up on explanation; we need only give up on reductive explanation. The possibility of explaining consciousness nonreductively remains open. This would be a very different sort of explanation, requiring some radical changes in the way we think about the structure of the world. ${ }^{39}$

Was zu guter Letzt das Problem der explanatorischen Lücke noch schwieriger machen könnte, ist die neurophysiologische Tatsache, dass es sozusagen keine Unterschiede bei der durch einen Reiz ausgelösten Form der Aktionspotenziale (den spikes) gibt. Die neuronalen Prozesse scheinen immer gleich zu sein, selbst wenn sie mit den unterschiedlichsten Formen von Erleben einhergehen. Das bedeutet, dass die durch einen Reiz ausgelösten Formen der spikes, bspw. im visuellen und im auditorischen System, identisch sind. So ist es erstaunlicherweise unmöglich, allein aus der Frequenz oder Form der spikes die Zugehörigkeit eines aktivierten Neurons zu einem bestimmten Sinnessystem zu eruieren..$^{40}$ Erst wenn man genauere Kenntnis über den Gehirnort eines aktivierten Neurons hat, weiss man, dass das Neuron z.B. zum visuellen System gehört:

Hirnforscher behaupten, es gebe zwischen den neuronalen Hirnzuständen und den bewusst erlebten oder mentalen Zuständen eine eindeutige Beziehung. Das würde bedeuten, dass man aus der Kenntnis von Hirnprozessen mehr oder weniger eindeutig auf mentale Prozesse schließen kann und eventuell auch

$36 \quad$ Vgl. a.a.O., S. 28.

37 Ebd.

38 Searle, John R. / [Übers.: Gavagai, Harvey]: Die Wiederentdeckung des Geistes, München: Artemis-Verlag \& Winkler [zuerst 1992, engl.]/1993, S. 26. Searle zitiert nur diese Konzeption. Nach ihm ist eine Realitätskonzeption in der es »keinen Platz« für Bewusstsein hat, nicht nur falsch sondern sogar »völlig unwissenschaftlich«. A.a.O., S. 27.

39 Chalmers, David J.: The conscious mind. In search of a fundamental theory, New York: Oxford University Press 1996, S. 122 [Hervorhebung E. E.].

40 Vgl. Roth, Gerhard: Das Gehirn und seine Wirklichkeit. Kognitive Neurobiologie und ihre philosophischen Konsequenzen, Frankfurt am Main: Suhrkamp 1994, S. 22. 
umgekehrt. Erforsche ich aber das Gehirn, dann entdecke ich nur feuernde Neurone und ausgeschüttete Neurotransmitter, aber keinerlei Wahrnehmungsinhalte, d.h. keine Farben, Formen, Töne, Gerüche; auch keine Denkvorgänge, Erinnerungen, Gefühle. Außerdem - und das ist noch gravierender - scheint es überhaupt keine Unterschiede in den neuronalen Prozessen zu geben, die in den verschiedenen Hirnarealen ablaufen und etwa mit Farbwahrnehmung oder mit Tonwahrnehmung zu tun haben, mit Körperbewegung oder mit Gesichtererkennung. [...] Kann die Vielfalt meiner Sinneswahrnehmungen überhaupt etwas mit der eintönigen Sprache der Neurone< zu tun haben? ${ }^{41}$

\subsubsection{Epistemische Argumente: Physikalisches Wissen als unzureichend für phänomenales Wissen}

Epistemische Argumente, die nach Brian Loar zu den einflussreichsten Gedanken von ReduktionsskeptikerInnen gehören, ${ }^{42}$ versuchen erstens aufzuzeigen, dass physikalisches Wissen nicht identisch mit phänomenalem Wissen ist. Ganz allgemein ausgedrückt, zeigen epistemische Argumente auf, dass eine Diskrepanz zwischen zwei Wissensformen besteht: »We can appeal to epistemology, arguing that the right sort of link between knowledge of physical facts and knowledge of consciousness is absent «. 43

Aus dieser Diskrepanz kann zweitens insofern auf eine epistemische Lücke geschlossen werden, als selbst vollständiges physikalisches Wissen nicht hinreichend ist, um einen epistemischen Zugang zum Phänomenalen zu gewährleisten. Auf diese Weise argumentiert das durch Frank Jackson berühmt gewordene Argument des unvollständigen Wissens (»knowledge argument «), ${ }^{44}$

\footnotetext{
41 Ebd.

42 Vgl. Loar, Brian: »Phenomenal states«, in: Philosophy of mind. Critical concepts in philosophy. Volume 4: Consciousness, Crawford, Sean [Hrsg.], London: Routledge \& Kegan Paul [zuerst 199o]/2011, S. 48. Loar selbst spricht diesbezüglich vom »[...] the most influential current anti-physicalist line of thougt $[\ldots] \ll$. Ebd. Dies ist jedoch zu plakativ, weil wir auch nicht-reduktionistische und reduktionistische Formen des Physikalismus unterscheiden müssen. Vgl dazu auch Chalmers, David J.: The conscious mind. In search of a fundamental theory, New York: Oxford University Press 1996, S. 128.

43 A.a.O., S. 93-94.

44 Vgl. Jackson, Frank: »What Mary Didn't Know«, The Journal of Philosophy, [1986] Vol. 83, No. 5, S. 291-295.
} 
das seinerseits auf von Locke ${ }^{45} 1689$ und Russell ${ }^{46} 1927$ formulierten Überlegungen basiert. ${ }^{47}$

In den Versuchen, epistemische Argumente zu entkräften, finden wir wiederum Einwände, die auf deflationären Begriffsdeutungen bzw. Bedeutungsverschiebungen beruhen und deshalb an die oben erwähnten deflationären Verwendungen des Erklärungsbegriffes erinnern: entweder wird der epistemische Wert von phänomenalem Wissen angezweifelt ${ }^{48}$ oder phänomenales Wissen wird neu als »Fähigkeit «49 gedeutet. Wiederum wird also versucht, ein Argument zu entkräften, indem ein Begriff, der im Argument eine zentrale Rolle spielt, deflationär verwendet bzw. relativiert wird: wenn phänomenales Wissen keinen oder keinen relevanten epistemischen Wert hat, dann erscheint die Unzulänglichkeit von physikalischem Wissen, Phänomenales erfassen zu können, offenbar nur noch halb so oder gar nicht mehr problematisch. ${ }^{50}$

45 Bei Locke ist die Vorlage für das knowledge argument besonders ostensiv: $»[\ldots]$ wenn ein Kind, bis es erwachsen wäre, an einem Orte festgehalten würde, wo es nie etwas anderes sähe als die Farben schwarz und weiss, [würde] es als Erwachsener ebenso wenig eine Idee von scharlachrot oder grün haben [...], wie jemand eine Idee von dem eigentümlichen Wohlgeschmack der Auster oder der Ananas besitzt, der von Kindheit her nie dergleichen gekostet hat «. Locke, John / [Hrsg.: Brandt, Reinhard]: Versuch über den menschlichen Verstand. Band I: Buch I und II, Hamburg: Meiner [zuerst 169o, engl.]/20o6, S. 111.

46 Russell verweist darauf, dass ein Blinder die gesamte Physik kennen könnte und dennoch bestimmte Dinge nicht wüsste, die Sehende wissen. Vgl. Russell, Bertrand: The analysis of matter, London: Kegan Paul, Trench, Truber \& Co 1927, S. 389 .

47 Mary ist eine Wissenschaftlerin, die schon immer einem schwarz-weissen Raum gelebt hat und trotz ihres vollständigen physikalischen Wissens über Farben, kein phänomenales Wissen über Farben hat. Vgl. Jackson, Frank: »What Mary Didn’t Know«, The Journal of Philosophy, [1986] Vol. 83, No. 5, S. 291-295. Vgl. dazu auch Walter, Sven: »Allgemeine Einleitung: Phänomenales Bewusstsein: Unlösbares Mysterium oder seriöses wissenschaftliches Problem?«, in: Qualia. Ausgewählte Beiträge, Heckmann, Heinz-Dieter / Walter, Sven [Hrsg.], Paderborn: Mentis 2001, S. 34.

48 Vgl. Metzinger, Thomas: Subjekt und Selbstmodell. Die Perspektivität phänomenalen Bewusstseins vor dem Hintergrund einer naturalistischen Theorie mentaler Repräsentation, Paderborn: Mentis 1999, S. 41.

49 Lycan, William G. [Hrsg.]: Mind and cognition. An anthology, Malden, Massachusetts: Blackwell 1999, S. 459.

50 Analog dazu liesse sich zur oben dargelegten deflationären Verwendungsweise des Erklärungsbegriff sagen: wenn `Erklärung` nur noch bedeutet, dass eine Korrelation von bestimmten Vorgängen (z.B. C-Faser) aufgezeigt werden kann, die mit dem zu erklärenden Phänomen (z.B. Schmerz) einhergehen, dann haben wir auch keine Erklärungslücke mehr. Weil `Erklärung〈, wenn sie deflationär gedeutet wird, nur noch im Nachweis einer Korrelation und in einer damit verbundenen (d.h. a priori vorausgesetzten) Identifikation des Feuerns von C-Fasern mit dem Phänomen `Schmerz bestehen würde. 
[...] knowing what an experience is like just is the possession of these abilities to remember, imagine, and recognize. It isn't the possession of any kind of information, ordinary or peculiar. [...] It isn't knowing-that. It's knowing-how. ${ }^{51}$

Doch selbst wenn wir Lycan diese epistemologisch deflationäre Bedeutungstransformation von phänomenaler Farbinformation als blosse »Fähigkeit «52 einmal einfach abnehmen, so ist damit das Problem, das im Argument von Jackson zum Ausdruck kommt, noch nicht gelöst: eine Farbwahrnehmung bleibt auch mit einem deflationären Wissensbegriff und noch so viel physikalischem Wissen unzugänglich. Selbst wenn eine Farbwahrnehmung als >kein Wissen oder als blosses $\gg$ knowing-how « ${ }^{53}$ gedeutet wird, so ist immer noch nicht klar, warum physikalisches Wissen keinen Zugang zu diesem Phänomen ermöglicht - unabhängig davon, ob es sich nun um eine Fähigkeit oder um ein >Wissen $<$ handelt. Die semantische Debatte über verschiedene Verwendungen des Wissensbegriffs ist diesbezüglich zweitrangig, denn das hard problem, bzw. die Kluft zwischen dem phänomenalen Zugang zu einem Erlebnis und dem physikalischem Wissen über Erlebniskorrelate, bleibt bestehen, unabhängig davon, wie $>$ Wissen $<$ nun definiert wird. ${ }^{54}$

Die Tatsache, dass Knut Nordby, ein bekannter norwegischer Wahrnehmungspsychologe und Spezialist für visuelle Wahrnehmung, der seit seiner Geburt vollständig Farbenblind war, keine Farben erleben kann, egal wie viel er über sie weiss, bleibt bestehen, selbst wenn das Farbensehen kein >Wissen<

$51 \quad$ Ebd.

52 Ebd.

53 Ebd.

54 Im Rahmen dieser Arbeit ist es zwar nicht möglich, nähre auf die Debatte einzugehen, inwiefern phänomenales Erleben nun ein Wissen im engeren Sinne ist oder nicht. Phänomenale Bewusstseinszustände haben jedoch insofern eine epistemische Relevanz, als nicht-propositionale Wissensformen, z.B. wie eine Farbe phänomenal erscheint, erfahrungsgemäss durch propositionales (bzw. physiologisches) Wissen alleine nicht zugänglich sind. Selbst eine negative Beantwortung der Frage, ob nicht-propositionale Zustände im engeren Sinne ein >Wissen < darstellen, würde nicht das Problem auflösen, dass das Erleben von Farben nicht alleine durch propositionales bzw. physiologisches Wissen über Sehvorgänge epistemisch zugänglich ist. Mehr zu nicht-propositionalen Zuständen und phänomenalem Bewusstsein, vgl Schildknecht, Christiane: Aspekte des Nichtpropositionalen, Bonn: Bouvier 1999, S. 11. Die Konzeption von nicht-propositionalem Wissen lässt sich auch mit Russells Begriff des knowledge by aquaintance vergleichen; vgl. dazu Russell, Bertrand: »Knowledge by acquaintance and knowledge by description«, in: Philosophy of mind. Critical concepts in philosophy. Volume 1: Foundations, Crawford, Sean [Hrsg.], London: Routledge \& Kegan Paul [zuerst 1910]/2011, S. 3o. Propositionales Wissen kann, innerhalb dieser Terminologie, demgegenüber als knowledge by Description bezeichnet werden; vgl. dazu a.a.O., S. 33 und 35 . 
im üblichen Sinne darstellen würde. ${ }^{55}$ Aufgrund seiner präzisen Schilderungen der prinzipiellen epistemischen Unzugänglichkeit, ${ }^{56}$ die ihn durch seine Farbenblindheit direkt betroffen hat, kann es auch nicht überzeugen, wenn Reduktionsoptimisten ${ }^{57}$ behaupten, die Unterscheidung von visuellem Erleben und damit verbundenen physiologischen Prozessen sei eine »vortheoretische Konstruktion $\ll^{58}$ bzw. basiere auf »Intuitionspumpen $\ll^{59}$ oder sei - im Gegensatz zu empirischen Befunden - bloss eine »Armchair-Analyse «.60

Beide obigen Begriffe der Einschüchterung ${ }^{61}$ gegen Reduktionsskeptiker, sind beim Fallbeispiel Nordby, dessen Lage in epistemischer Hinsicht derjenigen von Mary in Jacksons Gedankenexperiment gleicht, ${ }^{62}$ nicht anwendbar: 1.) Es geht bei der Feststellung, dass jemand mit physikalischem Wissen über Farben keinen Zugang zu Farbwahrnehmung hat, nicht einfach um

55 Vgl. dazu Nordby, Knut: »Vision in a complete achromat: a personal account«, in: Night vision. Basic, clinical, and applied aspects, Hess, Robert / Sharpe, Lindsay / Nordby, Knut [Hrsg.], Cambridge, Massachusetts: Cambridge University Press 199o, S. 297-305.

56 Vgl. ebd.

57 Als >Reduktionsoptimisten< bezeichne ich Vertreter, die von der Metaphysik des materialistischen Monismus überzeugt sind, und die im Hinblick auf das hard problem optimistisch sind, dass sich Erleben vollständig auf (dem heutigen Verständnis entsprechende) physikalischer Vorgänge reduzieren lässt.

$5^{8}$ Dennett, Daniel C.: »Quining Qualia«, in: Philosophy of mind. Classical and contemporary readings, Chalmers, David J. [Hrsg.], New York: Oxford University Press [zuerst 1988]/2002, S. 229.

59 Dennett verwendet den Begriff der »Intuitionspumpen« im Kontext seines Versuches, die »traditionelle« Konzeption von Qualia zu widerlegen: »What follows is a series of fifteen intuition pumps [...] designed to flush out - and then flush away - the offending intuitions. In section 2, I will use the first two intuition pumps to focus attention on the traditional notion. It will be the burden of the rest of the paper to convince you that these two pumps, for all their effectiveness, mislead us and should be discarded «. A.a.O., S. 227.

6o Mandik sagt zwar nicht explizit, dass Jacksons Gedankenexperiment eine »ArmchairAnalyse« sei. Sein Vorwurf der »Armchair-Analyse« scheint sich eher generell gegen Reduktionsskeptiker zu wenden: »But Quine has given us good reason to be skeptical about the deliverances of armchair analyses. [...] It may be that empirical results in the brain sciences will alter our naïve conception of consciousness [...] «. Mandik, Pete / Weisberg, Josh: »Type-q materialism «, 2008, Online: http://www.petemandik.com/ philosophy/papers/typeq.pdf [zuletzt geprüft am 13.09.2018], S. 20.

61 Mehr über die von mir gefundene Praxis der Einschüchterung von Reduktionsskeptikern, vgl. Abschnitt 4.2.4.

62 Folgende Schilderung von Nordby erinnert noch stärker an das Gedankenexperiment mit Jacksons Mary:»[...] red roses on bushes I see best in the twilight when I can separate the >black flowers from the >grey< leaves«. Nordby, Knut: »Vision in a complete achromat: a personal account «, in: Night vision. Basic, clinical, and applied aspects, Hess, Robert / Sharpe, Lindsay / Nordby, Knut [Hrsg.], Cambridge, Massachusetts: Cambridge University Press 1990, S. 308. 
eine »Intuitionspumpe«. Der Befund über diese epistemischen Lücke kann sich auch auf Berichte, wie die des Wahrnehmungsspezialisten Knut Nordby stützen. ${ }^{63}$ Der Verweis auf eventuelle zukünftige Erkenntnisse, die es möglich machen könnten, dass Farberlebnisse >irgendwie< und >irgendwann < durch physikalisches Wissen >objektiv< erfasst werden könnten, erinnert demgegenüber viel stärker an eine Intuition. ${ }^{64}$ 2.) Der Bericht von Nordby ist auch nicht das Resultat irgendwelcher »Armchair-Analysen ${ }^{6}{ }^{65}$ sondern eine detaillierte und empirisch auch auf Berichte anderer Farbenblinder rekurrierende Beschreibung einer epistemischen Diskrepanz. ${ }^{66}$ Der epistemische Zugang zum phänomenalen Gehalt >Farben< war Nordby, trotz seines professionellen, wahrnehmungsphysiologischen Wissens über Farben, vollständig verwehrt.

[...] I only see the world in shades that colour-normals describe as black, white and grey. [...] I experience the colour called red as a very dark grey, nearly black, even in very bright light. [...] Although I have acquired a thorough theoretical knowledge of the physics of colours and the physiology of the colour receptor mechanisms, nothing of this can help me to understand the true nature of colours. ${ }^{67}$

Das Problem, das sich nun jemand einhandelt, die oder der darauf besteht, dass das epistemische Argument, nicht aufzeige, dass der Physikalismus unvollständig ist, besteht darin, dass irgendwie aufgezeigt werden muss, dass physiologisches Wissen eben doch einen Zugang zu phänomenalem Erleben ermöglichen könnte:

63 Vgl. a.a.O., S. $290-315$.

64 Diese Intuition bzw. Hoffnung findet sich bspw. bei Churchland. Vgl. dazu Churchland, Paul M.: »The Rediscovery of Light«, The Journal of Philosophy, [1996] Vol. 93, No. 5, S. 227.

65 Mandik, Pete / Weisberg, Josh: »Type-q materialism«, 2008, Online: http://www. petemandik.com/philosophy/papers/typeq.pdf [zuletzt geprüft am 13.09.2018], S. 20.

66 Nordby bezieht sich in seinen Darlegungen auch auf die Berichte anderer farbenblinder Personen die die epistemische Unzugänglichkeit zu Farben scheinbar ähnlich wie er erleben, jedoch keine Wahrnehmungsspezialisten sind.: »Most achromatopic people think of color as some curious property of surfaces that for them is somehow related to their apparent brightness. Thus yellow looks lighter than other colors, and red loods darker«. Nordby, Knut: »What is this thing you call color: can a totally color-blind person know about color?«, in: Phenomenal concepts and phenomenal knowledge. New essays on consciousness and physicalism, Alter, Torin / Walter, Sven [Hrsg.], Oxford: Oxford University Press 2007, S. 79. Zu seinen Berichten über das Erleben anderer farbenblinder Personen, vgl. a.a.O., S. 78, 79 und 81-82.

67 Nordby, Knut: »Vision in a complete achromat: a personal account«, in: Night vision. Basic, clinical, and applied aspects, Hess, Robert / Sharpe, Lindsay / Nordby, Knut [Hrsg.], Cambridge, Massachusetts: Cambridge University Press 199o, S. 305. 
If ultimately they [Qualia] are physical, then inner qualia ought to be epistemically accessible from more than just the first-person or >subjective< point of view; they ought to be accessible as well from one or more >objective< points of view, via some appropriate instruments that scan brain activity, for example. ${ }^{68}$

Weil dieser Zugang nicht aufgezeigt werden kann, besteht die vielleicht letzte Möglichkeit noch darin, auf eine Zukunft zu verweisen, in der »mit den richtigen Messinstrumenten « ${ }^{69}$ das subjektive phänomenale Erleben einer Person aus einer oder mehreren »objektiven Perspektiven ${ }^{70}{ }^{70}$ zugänglich sein könnte. Eine Antwort auf die Frage, wie das auch nur im Prinzip möglich sein könnte, bleibt uns Churchland jedoch schuldig.

Eine andere Variante der Versuche, das epistemische Argument zu entkräften, besteht in der auch beim explanatorischen Argument angewandten ${ }^{71}$ Konzeption der phänomenalen Begriffe, die nach Mandik die Funktion haben, die phänomenalen Aspekte von Wahrnehmungen herauszuschälen. ${ }^{72}$ Die Konzeption der phänomenalen Begriffe gründet auf einer Kritik an (u.a. aus epistemischen Argumenten abgeleiteten) Nicht-Begrifflichkeits-Konzeptionen von Qualia. Diese Kritik geht davon aus, dass nur ein Gehalt, der begrifflich ist, eine Überzeugung ${ }^{73}$ erwirken könne. ${ }^{74}$ Demgemäss können auch nach Tye Qualia nur bewusst erlebt werden, wenn wir über sie einen phänomenalen Begriff verfügen. ${ }^{75}$

68 Churchland, Paul M.: »The Rediscovery of Light«, The Journal of Philosophy, [1996] Vol. 93, No. 5, S. 227 [Hervorhebung EE; Ergänzung in Klammern E.E.]

69 (wörtlich:»via some appropriate instruments«) Ebd.

70 (wörtlich:»from one or more >objectiveく points of view«) Ebd.

71 Vgl. Chalmers, David J.: »Phenomenal concepts and the explanatory gap«, in: Phenomenal concepts and phenomenal knowledge. New essays on consciousness and physicalism, Alter, Torin / Walter, Sven [Hrsg.], Oxford: Oxford University Press 2007, S. 167.

72 Vgl. Mandik, Pete / Weisberg, Josh: »Type-q materialism«, 2008, Online: http://www. petemandik.com/philosophy/papers/typeq.pdf [zuletzt geprüft am 13.09.2018], S.19. Nach Loar sind phänomenale Begriffe als Wiedererkennungsbegriffe zu verstehen. Vgl. dazu Pauen, Michael: »Warum die Autorität der ersten Person das Erklärungslückenargument untergräbt«, in: Den eigenen Geist kennen. Selbstwissen, privilegierter Zugang und Autorität der ersten Person, Newen, Albert / Vosgerau, Gottfried [Hrsg.], Paderborn: Mentis 2005, S. 220.

73 Bspw. die Überzeugung, dass wir etwas Violettes sehen.

74 Mehr über Diskrepanz zwischen phänomenalen, nicht-begrifflichen Wahrnehmungsgehalten und begrifflichen Überzeugungsgehalten und über Nicht-BegrifflichkeitsKonzeptionen von Qualia, vgl. Schildknecht, Christiane: »Komplementäre Sichtweisen des 〉Gegebenen««, in: Genese und Geltung: Für Gottfried Gabriel, Schildknecht, Christiane / Teichert, Dieter / Van Zantwijk, Temilo [Hrsg.], Paderborn: Mentis 20o8, S. 100.

75 Vgl. Tye, Michael: »Das Problem primitiver Bewusstseinsformen: Haben Bienen Empfindungen?«, in: Bewusstsein und Repräsentation, Esken, Frank / Heckmann, HeinzDieter [Hrsg.], Paderborn: Mentis [zuerst 1997, engl.]/1999, S. 121. 
Eine Konzeption von Erleben als blosse Form einer Überzeugung wird dem Phänomenalen aber nicht gerecht. ${ }^{76}$ Schmerz wird von uns auch erlebt, wenn wir nicht jeden Moment denken sich bin der Meinung, dass ich Schmerzen habe ${ }^{77}$ Das heisst, Schmerz ist auch ohne eine begriffliche Repräsentation fühlbar. ${ }^{78}$ Umgekehrt, kann ein sprachlich überaus versierter Farbenblinder wie z.B. Nordby, einen Test seiner Überzeugungen zum Thema >Violett< zwar bestehen (d.h., er könnte diesem Farbbegriff viele Objekte mit jener Eigenschaft zuordnen). Dennoch hat er nicht die (phänomenale) Information, wie Gegenstände, die violett sind, aussehen. Nur ein visuelles Farberlebnis, könnte ihm diese Information geben. ${ }^{79}$ Was ebenfalls gegen die Begrifflichkeitskonzeption

76 Vgl. dazu Schildknecht, Christiane: »Klarheit in Philosophie und Literatur. Überlegungen im Anschluss an Peter Bieri«, Deutsche Zeitschrift für Philosophie, [2008] Vol. 56, No. 5, S. 783 .

77 Aufgrund dieser Kritik gehen bspw. dispositionalistische Varianten der HOT-Theorien davon aus, dass die Disposition, einen höherstufigen Gedanke bzw. eine Überzeugung zu einem bestimmten Wahrnehmungsgehalt bilden zu können, für das bewusste Erleben einer Wahrnehmung genügt. Vgl. dazu Carruthers, Peter: »Higher-Order Theories of Consciousness«, in: The Stanford Encyclopedia of Philosophy, 2016, Online: https:// plato.stanford.edu/archives/fall2016/entries/consciousness-higher/ [zuletzt geprüft am 25.07.2017], Abschnitt 5.0. Vgl. ebenfalls Carruthers, Peter: Consciousness. Essays from a higher-order perspective, Oxford: Clarendon Press 2005. Dies löst aber nicht das vom epistemischen Argument aufgeworfene Problem, denn auch ein dispositionaler phänomenaler Begriff bzw. die Disposition, eine Überzeugung zu einem bestimmten Wahrnehmungsgehalt bilden zu können, muss irgendwann einmal durch ein phänomenale Erlebnis erlernt worden seien. Das heisst, auch ein dispositionaler, phänomenaler Begriff kann nicht alleine aus propositionalen Begriffen bestehen oder durch sie angeeignet werden, weil er nur durch ein phänomenales Erlebnis (bzw. durch ein mit dem Erlebnis verbundenen nicht-propositionalen Wissen) gebildet werden kann. Vgl. dazu auch Stubenberg, Leopold: Consciousness and qualia, Amsterdam: John Benjamins 1998, S. 6o.

78 Vgl. dazu Lanz, Peter: Das phänomenale Bewusstsein. Eine Verteidigung, Frankfurt am Main: Klostermann 1996, S. 202.

79 Nordby beispielsweise hatte schon als Kind gelernt Farbbegriffe anzuwenden, aber dies half ihm nicht im Geringsten, Farben tatsächlich zu erleben. Schon in seiner Zeit als Primarschüler machte er die Erfahrung, dass andere Personen bzw. Normalsichtige visuelle Eigenschaften von Oberflächen erkennen. Die mit diesen Erlebnissen offenbar zusammenhängenden und von anderen kommunizierten Farbbegriffen konnte er zwar anwenden, jedoch überhaupt nicht begreifen, was damit gemeint sein könnte: »As an aid for teaching the letters of the alphabet, the teacher placed large cards, each holding a printed letter, in a row over the blackboard as the letters were introduced. To differentiate between the two categories of letters they had different colours; the vowels were red, while the consonants were black. I could not see any difference between them and could not understand what the teacher meant, until early one morning late in the autumn when the room-lights had been turned on, and, unexpectedly, I saw that some of the letters, i.e. the A E I U Y Å Ä Ö, were now suddenly a darkish grey, while the others were still solid black«. Nordby, Knut: »Vision in a complete achromat: a personal account«, in: Night vision. 
von Qualia spricht, ist die Tatsache, dass Wahrnehmungsgehalte von phänomenalem Erleben, die wir visuell unterscheiden können, (z.B. bei den farblichen Graduierungen) sehr viel feinkörniger und differenzierter sind, als unser begriffliches Instrumentarium dafür ist. ${ }^{80}$

Wird die mit sprachlichen Begriffen nicht zu fassende Feinkörnigkeit und Phänomenalität von Erleben eingestanden, besteht eine weitere Variante, Phänomenales als etwas Begriffliches zu deuten noch in der Konzeption so genannter $>$ demonstrativer ${ }^{81}$ bzw. >indexikalischer Begriffe.$^{82}$ Doch der begriffliche Gehalt demonstrativer Ausdrücke ist viel zu unterdeterminiert um mit ihm Phänomenales epistemisch erfassen zu können: wenn jemand Knut Nordby mit den Worten >dies ist rot eine Farbwahrnehmung bescheren wollte, wäre er damit sicher nicht erfolgreich. ${ }^{83}$

Die Konzeption von Qualia als indexikalische Begriffe ${ }^{84}$ oder phänomenale Begriffe $^{85}$ gründet ebenfalls auf einer Bedeutungsverschiebung: phänomenales Erleben und auf begriffliche Wissensformen nicht reduzierbare Wahrnehmungsgehalte ${ }^{86}$ werden als $»$ Begriffe $\ll^{87}$ umgedeutet, die einfach einem

Basic, clinical, and applied aspects, Hess, Robert / Sharpe, Lindsay / Nordby, Knut [Hrsg.], Cambridge, Massachusetts: Cambridge University Press 199o, S. 297 [Hervorhebung E.E.].

8o Vgl. dazu Schildknecht, Christiane: »Komplementäre Sichtweisen des >Gegebenen ««, in: Genese und Geltung: Für Gottfried Gabriel, Schildknecht, Christiane / Teichert, Dieter / Van Zantwijk, Temilo [Hrsg.], Paderborn: Mentis 2008, S. 97. Vgl. ebenfalls Staudacher, Alexander: Phänomenales Bewusstsein als Problem für den Materialismus, Berlin: De Gruyter 2002, S. 6. Vgl. ebenfalls Schildknecht, Christiane: »Klarheit in Philosophie und Literatur. Überlegungen im Anschluss an Peter Bieri«, Deutsche Zeitschrift für Philosophie, [2008] Vol. 56, No. 5, S. 783 .

81 Vgl. dazu ebd.

82 Vgl. Pauen, Michael / Schütte, Michael / Staudacher, Alexander [Hrsg.]: Begriff, Erklärung, Bewusstsein. Neue Beiträge zum Qualia-Problem, Paderborn: Mentis 2007, S. 16-19 und 22. Begriffe, wie >hier<, >jetzt<, >dies<, können als indexikalische Weisen der Bezugnahme gesehen werden. Mit dem Begriff >dies< können wir bspw. demonstrativ auf eine Farbe zeigen, ohne dass wir sie begrifflich benennen müssen.

83 Auf das epistemische Argument von Jackson angewandt hiesse das, dass das Fehlen von phänomenalem Wissen mehr ist, als das Fehlen von indexikalischem Wissen: »In lacking phenomenal knowledge, she [Mary] lacks far more than someone lacking indexical knowledge«. Chalmers, David J.: The conscious mind. In search of a fundamental theory, New York: Oxford University Press 1996, S. 144 [Ergänzung in Klammern von E.E.].

84 Vgl. Lycan, William G.: Consciousness and experience, Cambridge, Massachusetts: MIT Press 1996. Vgl. ebenfalls Pauen, Michael / Schütte, Michael / Staudacher, Alexander [Hrsg.]: Begriff, Erklärung, Bewusstsein. Neue Beiträge zum Qualia-Problem, Paderborn: Mentis 2007, S. 16-19 und 22.

85 Vgl. a.a.O., S. $16-17$.

86 Vgl. dazu Schildknecht, Christiane: »Phänomenales Erleben in Kunst und Literatur«, in: Kunst denken, Burri, Alex [Hrsg.], Paderborn: Mentis 2007, S. 98-99.

87 Vgl. Pauen, Michael / Schütte, Michael / Staudacher, Alexander [Hrsg.]: Begriff, Erklärung, Bewusstsein. Neue Beiträge zum Qualia-Problem, Paderborn: Mentis 2007, S. 16. 
»speziellen Charakter «8 ${ }^{88}$ häten. ${ }^{89}$ Diese Varianten, das epistemische Argument zu entkräften, bestehen deshalb im Versuch, die epistemische Diskrepanz zwischen nicht-begrifflichem, phänomenalem Erleben und physikalischem bzw. begrifflichem Wissen mit einer Bedeutungsverschiebung einzuebnen. ${ }^{90}$

88 Tye, Michael: »Qualia«, in: The Stanford Encyclopedia of Philosophy, 2007, Online: http:// plato.stanford.edu/archives/fall2007/entries/qualia/ [zuletzt geprüft am 25.09.2018], Abschnitt 5.0.

89 Vgl. dazu Pauen, Michael / Schütte, Michael / Staudacher, Alexander [Hrsg.]: Begriff, Erklärung, Bewusstsein. Neue Beiträge zum Qualia-Problem, Paderborn: Mentis 2007, S. 16-19 und 22 .

9o Ist diese epistemische Diskrepanz einmal eingestanden, kann man mit Churchland noch behaupten, dass Jackson und alle, die sein Argument überzeugend finden, einem Fehlschluss unterliegen: Weil man etwas auf zwei verschiedene Weisen wissen könne, gingen sie davon aus, dass dies zwei Dinge seien, über die man Wissen erwerbe: »It elevates two distinct modes of epistemic access [...]«. Churchland, Paul M.: »The Rediscovery of Light«, The Journal of Philosophy, [1996] Vol. 93, No. 5, S. 219.

Natürlich wäre dies ein falscher Schluss, wenn es sich tatsächlich um ein Objekt mit nur physikalischen Eigenschaften handeln würde, welches »auf zwei Weisen« gesehen werden kann - doch genau dies ist ja die Ausgangsfrage im epistemischen Problem phänomenalen Erlebens, kurz des Qualia-Problems, auf welches sich sowohl Churchland als auch Jackson beziehen. Churchland zeigt aber erstens in seinem Argument gar nicht auf, dass ein Violett-Erlebnis ausschliesslich eine physikalische Eigenschaft ist. Sein Argument basiert auf einer Apriori-Annahme, nämlich in der Annahme, dass eine RotWahrnehmung nur ein Objekt mit physikalischen Eigenschaften sei, welches »auf zwei Weisen « gesehen werden könne: »What Blind Mary is missing is one common form of knowledge about light: she lacks perceptual/discriminative knowledge of light. And yet, people who have such knowledge are accessing the very same features of reality that she is obliged to access in other ways. [...] They are different forms of knowledge; they operate with different representational >palettes inside Mary's brain. But they both represent, each in their own distinct way, one and the same entirely physical thing: light «. Ebd. [Hervorhebung E. E.]. Seine Behauptung, dass das phänomenale Rot-Erleben vollständig >identisch mit einem objektiven >Ding sei, leitet er offenbar aus einer damit in Verbindung gebrachten Analogie ab. Leider beruft er sich dabei auf eine schon im Grundsatz äusserst fragwürdige Analogie, nämlich derjenigen zwischen Licht, als einem physikalisch-objektiven Phänomen (bzw. Prozess) und dem Phänomen des Erlebens von Licht. Diese Analogie wäre für dieses Argument aber nur zulässig, wenn feststehen würde, dass Farb-Erleben identisch mit einem >Ding $<$ ist. Aber genau dies steht ja in Frage. Genau genommen unterliegt Churchland also einem Kategorienfehler, aufgrund dessen der Vorgang oder das Phänomen des Sehens von Licht mit dem Wahrnehmungsobjekt >Licht<, welches gesehen wird, vermengt wird. Mehr Argumente zu diesem Kategorienfehler von Churchland finden sich Abschnitt 6.4.

Zudem zeigt er nicht auf, dass ein Farberlebnis ausschliesslich darin bestehen soll, über die Oberflächeneigenschaften eines violetten Gegenstandes informiert zu sein. Letzteres ist, wie Lanz ausführlich aufzeigt, deshalb ein Fehlschluss, weil die phänomenale Struktur von Farbwahrnehmungen gar nicht mit den physikalischen Strukturen von Farboberflächen übereinstimmt. Vgl. Lanz, Peter: Das phänomenale Bewusstsein. Eine Verteidigung, Frankfurt am Main: Klostermann 1996, S. 159-195 sowie S. 228-229. Vgl. ebenfalls a.a.O., S. 228. Vgl. dazu auch Roth, Gerhard: Das Gehirn und seine Wirklichkeit. 
Fazit:Epistemische Argumente zeigen, unabhängig davon, ob phänomenales Erleben als >Fähigkeit< oder als >indexikalische< bzw. >phänomenale Begriffe < umgedeutet wird, eine Asymmetrie ${ }^{91}$ im Hinblick auf die möglichen $\mathrm{Zu}$ gänge zu physikalischen versus phänomenalen Explananda auf: Wissen über physikalische Eigenschaften ist epistemisch unzureichend um phänomenales Erleben zu erfassen bzw. um die mit physikalischen Eigenschaften und Vorgängen korrelierenden phänomenalen Gehalte zu erleben. Wenn ein bestimmter Wissenskomplex unzureichend ist, um etwas zu erfassen, so muss er - im Hinblick auf das Phänomen, das erfasst werden soll - auch unvollständig sein. Und genau dies will man ja mit epistemischen Argumenten aufzeigen.

The epistemic asymmetry associated with consciousness [...] tells us that no collection of facts about complex causation in physical systems adds up to a fact about consciousness. ${ }^{92}$

Jacksons Mary ist zwar (wenn wir reduktionistisch-physikalistische Positionen von der Beweislast befreien, zu zeigen, dass es sich bei phänomenalem Erleben in >Wirklichkeit< um physikalische Eigenschaften handelt) noch kein Argument,

Kognitive Neurobiologie und ihre philosophischen Konsequenzen, Frankfurt am Main: Suhrkamp 1994, S. 319 und 342. Auch der Neurowissenschafter Gerhard Roth konstatiert, dass nicht einmal so einfache Wahrnehmungen wie Farben als Abbildungen der Realität gesehen werden können. Vgl. ebd. Vgl. dazu auch Albertazzi, Liliana: »Qualitative perceiving «, Journal of Consciousness Studies, [2012] Vol. 19, No. 11-12, S. 14 und 22. Vgl. ebenfalls Schildknecht, Christiane: »Phänomenales Erleben in Kunst und Literatur«, in: Kunst denken, Burri, Alex [Hrsg.], Paderborn: Mentis 2007, S. 98. Oder: Blasche, Siegfried: »Qualität III (Neuzeit)«, in: Historisches Wörterbuch der Philosophie. Band 7: P-Q, Ritter, Joachim / Kranz, Margarita [Hrsg.], Darmstadt: Wissenschaftliche Buchgesellschaft 1971-2007, S. 1776. Oder: Frank, Manfred: »Gibt es eine >innere Wahrnehmung ??«, in: Den eigenen Geist kennen. Selbstwissen, privilegierter Zugang und Autorität der ersten Person, Newen, Albert / Vosgerau, Gottfried [Hrsg.], Paderborn: Mentis 2005, S. 56. Die Unhaltbarkeit der in Churchlands Behauptung vorausgesetzten Externalismusthese zeigt also auf, dass sich die Behauptung, ein Farberlebnis bestünde ausschliesslich darin, über bestimmte Oberflächeneigenschaften informiert zu sein, sich auch empirisch nicht stützen lässt. Mehr über die Externalismusthese und die strukturelle Differenz von phänomenalem Erleben und Oberflächeneigenschaften vgl. Abschnitt 7.2.3.

91 Vgl. dazu auch McGinn, Colin: Wie kommt der Geist in die Materie? Das Rätsel des Bewusstseins, München: Beck 2001, S. 62 und 66. Vgl. ebenfalls Chalmers, David J.: »Bewusstsein und sein Platz in der Natur«, in: Grundkurs Philosophie des Geistes. Band 1: Phänomenales Bewusstsein, Metzinger, Thomas [Hrsg.], Paderborn: Mentis [zuerst 2002, engl.]/2007, S. 126 .

92 Chalmers, David J.: The conscious mind. In search of a fundamental theory, New York: Oxford University Press 1996, S. 102-103. 
welches die Patt-Situation zu Gunsten der Reduktionsskeptiker auflöst. Viele Philosophen geben aber zu, dass es mindestens ein Argument gegen reduktive Erklärungen von Phänomenalem ist. ${ }^{93}$ Befreien wir reduktionistische Positionen aber nicht von der Beweislast, folgt aus epistemischen Argumenten sogar, dass ein ontologisch-reduktiver ${ }^{94}$ Physikalismus falsch ist. ${ }^{95}$

Je nach der Art einer reduktionsoptimistischen Behauptung sind epistemische Argumente ein schwerwiegendes Problem für Physikalisten: Wenn behauptet wird, dass mit dem Wissen über physikalische Eigenschaften und Vorgänge alles - auch phänomenales Erleben - epistemisch zugänglich ist und somit reduktiv erklärt werden kann, dann lässt sich daraus schliessen, dass die dieser Behauptung zugrunde liegende Konzeption von >Physik entweder unvollständig oder falsch ist. ${ }^{96}$ Dieses Problem könnte nach Chalmers eine »radikale« Modifikation nach sich ziehen, wie wir über die »physikalische Struktur der Welt $\ll^{97}$ denken.

\subsubsection{Modale Argumente: Funktionale Identität mit phänomenaler Divergenz}

Die Gemeinsamkeit von modalen und epistemischen Argumenten besteht darin, dass beide Argumenttypen aufzuzeigen versuchen, dass phänomenale Tatsachen weder aus physikalischen Tatsachen abgeleitet werden können, noch dass Phänomenales mit Physikalischem zwingend identisch sein muss. ${ }^{98}$ Modale Argumente basieren zudem auf der ebenfalls in der Erklärungslücke problematisierten Kontingenz ${ }^{99}$ von Korrelationen zwischen physikalischen

93 Vgl. a.a.O., S. 104.

94 Genauer: ein strikt reduktiver Physikalismus, der auf seiner metaphysischen Prämisse einer radikal monistischen Ontologie beharrt.

95 Mehr über das Problem der Beweislastabschiebung reduktionsoptimistischer Argumentationen, vgl. Abschnitt 4.2.3

96 Vgl. dazu auch Robinson, Howard: »Dualism«, in: The Stanford Encyclopedia of Philosophy, 2013, Online: http://plato.stanford.edu/archives/fall2013/entries/dualism/ [zuletzt geprüft am 25.09.2018], Abschnitt 5.1. Oder: Velmans, Max: »Reflexive Monism. Psychophysical Relations among Mind, Matter, and Consciousness«, Journal of Consciousness Studies, [2012] Vol. 19, No. 9-10, S. 162. Oder: Feser, Edward: »Qualia. Irreducibly Subjective but not Intrinsic«, Journal of Consciousness Studies, [2001] Vol. 8, No. 8, S. 6, 9 und 13. Vgl. ebenfalls Warner, Richard: Introduction: The Mind-Body Debate«, in: The mind-body problem. A guide to the current debate, Warner, Richard / Szubka, Tadeusz [Hrsg.], Cambridge, Massachusetts: Blackwell 1994, S. 8.

97 Chalmers, David J.: The conscious mind. In search of a fundamental theory, New York: Oxford University Press 1996, S. 122.

98 Vgl. dazu a.a.O., S. 140.

99 Vgl. Levine, Joseph:»Materialism and Qualia: The Explanatory Gap«, Pacific Philosophical Quarterly, [1983] Vol. 64, No. 4, S. 356. Vgl. ebenfalls Levine, Joseph:»Phenomenal concepts 
Tatsachen und phänomenalem Erleben. ${ }^{100}$ So erörtert Bieri, mit einem Gedankenexperiment von Leibniz, ${ }^{101}$ die unzureichende Plausibilität, ${ }^{102}$ dass Menschen aufgrund von Gehirnvorgängen überhaupt etwas erleben. ${ }^{103}$ Demnach sei es denkbar, dass Menschen ebenso gut nicht »den Schatten eines Erlebnisses «104 haben könnten. Im Gedankenexperiment von Leibniz stellen wir uns vor, dass ein menschliches Gehirn so stark vergrössert wäre, dass wir in ihm umhergehen könnten, wie in einer riesigen Fabrik. Bei diesem Rundgang ergreifen uns Zweifel über die Notwendigkeit des Zusammenhangs zwischen den Prozessen in diesem Riesenhirn und dem, was >in< ihm entstehen soll: ein erlebendes Subjekt.

Was uns wirklich beschäftigt, ist dieses: Wir können uns ohne weiteres vorstellen, dass hier drin alles genau so wäre wie es ist, ohne dass der Mensch, in dessen Kopf wir sind, auch nur den Schatten eines Erlebnisses hätte. Nichts an dem, was uns gezeigt worden ist, scheint es notwendig zu machen, dass da einer etwas erlebt: nicht die Art des Materials, nicht die Architektonik der Fabrik, nicht die chemischen Reaktionen, nicht die elektrischen Muster. Es dünkt uns in gewissem Sinn zufällig, dass da nun auch noch ein erlebendes Subjekt auftaucht $[\ldots]]^{105}$

and the materialist constraint«, in: Phenomenal concepts and phenomenal knowledge. New essays on consciousness and physicalism, Alter, Torin / Walter, Sven [Hrsg.], Oxford: Oxford University Press 2007, S. 145. Vgl. dazu auch Michel, Jan G.: Der qualitative Charakter bewusster Erlebnisse. Physikalismus und phänomenale Eigenschaften in der analytischen Philosophie des Geistes, Paderborn: Mentis 2011, S. 93-136.

100 Andererseits besteht nach Pauens Verständnis das modale Argument auch darin, mit der Vorstellbarkeit von "psychophysischen Dissoziationen«, zu untermauern, dass sich phänomenale Alltagsbegriffe (im Hinblick auf eine Erklärung phänomenaler Eigenschaften) funktional nicht operationalisieren lassen. Pauen, Michael: »Warum die Autorität der ersten Person das Erklärungslückenargument untergräbt «, in: Den eigenen Geist kennen. Selbstwissen, privilegierter Zugang und Autorität der ersten Person, Newen, Albert / Vosgerau, Gottfried [Hrsg.], Paderborn: Mentis 2005, S. 217.

101 Vgl. Leibniz, Gottfried W. / [Hrsg: Köhler, Heinrich]: Lehr-Sätze über die Monadologie, Frankfurt: Meyer [zuerst 1714, franz.] $/ 1720, \S 17$.

102 Vgl. dazu auch Levine, Joseph: »Materialism and Qualia: The Explanatory Gap«, Pacific Philosophical Quarterly, [1983] Vol. 64, No. 4, S. 355.

103 Vgl. Bieri, Peter: »Was macht Bewusstsein zu einem Rätsel?«, in: Grundkurs Philosophie des Geistes. Band 1: Phänomenales Bewusstsein, Metzinger, Thomas [Hrsg.], Paderborn: Mentis [zuerst 1994]/2007, S. 42.

104 Ebd.

105 Vgl. ebd. [Hervorhebung im Original]. 
Demgemäss argumentiert auch Levine, im Rückgriff auf die Modalargumentation Kripkes ${ }^{106}$ gegen psychophysische Identitätstheorien dafür, dass C-Faserreizung auch ohne einen damit einhergehenden Schmerz vorstellbar ist: »[...] it immediately becomes imaginable that there be C-fiber firings without the feeling of pain, and vice versa ${ }^{107}$

Einerseits wird aus der Nicht-Plausibilität, dass neuronale Erregungen überhaupt mit bewusstem Erleben einhergehen, die Vorstellbarkeit des Gegenteils ${ }^{108}$ begründet. Andererseits wird aus dieser Nicht-Plausibilität die Nicht-Ableitbarkeit von phänomenalem Erleben aufgrund von physikalischen Tatsachen gefolgert. Und aufgrund dieser Nicht-Ableitbarkeit, besteht, nach den Verteidigern des modalen Argumentes, ebenfalls die (logische ${ }^{109}$ Möglichkeit, dass Phänomenales von physikalischen Tatsachen generell abweichen könnte.

We can think about what is conceivable, in order to argue directly for the logical possibility of a situation in which the physical facts are the same but the facts about experience are different. ${ }^{110}$

Dies wiederum bedeutet, dass phänomenales Erleben und neurologische Korrelate nicht zwingend identisch sein müssen. ${ }^{111}$ So plädiert ein bekanntes modales Argument von Chalmers dafür, dass die Denkbarkeit von mit uns

106 Kripke geht davon aus, dass bei allen wahren Identitätsbehauptungen auf beiden Seiten des Identitätsprädikats (z.B. >Wasser ist identisch mit $\mathrm{H}^{2} \mathrm{O}<$ ) starre Designatoren stehen müssen. Auf beiden Seiten des Identitätsprädikats bzw. der Identitätsbehauptung `Schmerz ist identisch mit dem Feuern von C-Fasern<, handelt es sich jedoch nicht um starre Designatoren, denn Schmerz muss nicht zwingend in einer C-Faserreizung realisiert sein. Behauptungen über psycho-physische Identitäten sind nach Kripke folglich falsch. Vgl. Kripke, Saul A.: Naming and necessity, Oxford: Blackwell [zuerst 1972]/1980, S. 97-105. Vgl. dazu auch Searle, John R. / [Übers.: Gavagai, Harvey]: Die Wiederentdeckung des Geistes, München: Artemis-Verlag \& Winkler [zuerst 1992, engl.]/1993, S. 55.

107 Levine, Joseph: »Materialism and Qualia: The Explanatory Gap«, Pacific Philosophical Quarterly, [1983] Vol. 64, No. 4, S. 359 [Hervorhebung im Original]. Vgl. dazu auch Michel, Jan G.: Der qualitative Charakter bewusster Erlebnisse. Physikalismus und phänomenale Eigenschaften in der analytischen Philosophie des Geistes, Paderborn: Mentis 2011, S. $93^{-136 .}$

108 Das heisst: neuronale Erregungen ohne bewusstes Erleben.

109 In der Diskussion über modale Argumenttypen ist von drei Modalitäten die Rede: 1. logische bzw. begriffliche Möglichkeit; 2. metaphysische Möglichkeit; 3. nomologische Möglichkeit. Mehr dazu vgl. weiter unten.

110 Chalmers, David J.: The conscious mind. In search of a fundamental theory, New York: Oxford University Press 1996, S. 93.

111 Vgl. Kripke, Saul A.: Naming and necessity, Oxford: Blackwell [zuerst 1972]/198o, S. 97-105. Vgl. dazu auch Michel,Jan G.: Der qualitative Charakter bewusster Erlebnisse. Physikalismus 
funktional identischen Menschen, die aber kein bewusstes Erleben haben (»Zombies«), ${ }_{112}^{12}$ dafür spricht, dass physikalische Vorgänge nicht mit Qualia identisch sein könnten. ${ }^{113}$ Ebenfalls auf der Denkbarkeit von fehlendem phänomenalen Erleben, bei identischer funktionaler Organisation, basiert Blocks Gedankenexperiment der so genannten »verrückten « Realisierung. ${ }^{114}$ Eine andere Variante des modalen Argumentes, die ursprünglich auf Locke ${ }^{115}$ zurückgeht, argumentiert dafür, dass es denkbar ist, dass funktional identische

und phänomenale Eigenschaften in der analytischen Philosophie des Geistes, Paderborn: Mentis 2011, S. 93-136.

112 Vgl. Chalmers, David J.: »Zombies on the web«, 2017, Online: http://consc.net/zombies. html [zuletzt geprüft am 13.09.2018]. Vgl. ebenfalls Beckermann, Ansgar: Analytische Einführung in die Philosophie des Geistes, Berlin: W. de Gruyter 2008, S. 168 und 442. Nach Block könnte zwar auch ein totaler Zombie Bewusstsein haben, wenn man einen anderen Sinn des Wortes >Bewusstsein < in Erwägung ziehe, was wiederum an die oben erwähnten Bedeutungsverschiebungen erinnert. Im modalen Argument des Zombies geht es aber um einen Bewusstseinsbegriff der zwingend bewusstes Erleben impliziert. Vgl. dazu die diesbezügliche Kritik an Block von Searle, John R. / [Übers.: Gavagai, Harvey]: Die Wiederentdeckung des Geistes, München: Artemis-Verlag \& Winkler [zuerst 1992, engl.]/1993, S. 103 .

113 Vgl. Chalmers, David J.: The conscious mind. In search of a fundamental theory, New York: Oxford University Press 1996, S. 94-99.

114 In ihm stellt sich Block vor, wie z.B. die chinesische Nation die Funktion eines Hirns simulieren könnte. Ein solches System hätte plausiblerWeise - so sein modales Argumenttrotz postulierter Funktionsisomorphie mit einem Hirn wohl kaum ein phänomenales (Gruppen-) Bewusstsein. Vgl. Block, Ned J.: »Troubles with Functionalism«, in: Readings in philosophy of psychology, Block, Ned J. [Hrsg.], London: Methuen [zuerst 1978]/1980. Vgl. dazu auch Loar, Brian: »Phenomenal states«, in: Philosophy of mind. Critical concepts in philosophy. Volume 4: Consciousness, Crawford, Sean [Hrsg.], London: Routledge \& Kegan Paul [zuerst 199o]/2011, S. 62. Vgl. ebenfalls Levin, Janet: »Functionalism«, in: The Stanford Encyclopedia of Philosophy, 2013, Online: http://plato.stanford.edu/archives/ fall2013/entries/functionalism/ [zuletzt geprüft am 28.o9.2018], Abschnitt 5.5.1.

Nach Chalmers ist das Gedankenexperiment von Block, gerade weil es sich um eine sehr unübliche Realisation der funktonalen Organisation eines menschlichen Hirns handelt, indirekt ein Argument für die logische Möglichkeit von Zombies. So verwendet er die Plausibilität, dass China, auch wenn es funktional ein Hirn simulieren würde, kein "phänomenalen Gruppenbewusstseins« (»group mind«) hätte, als Argument für die Möglichkeit von Zombies:»[...] it follows that the existence of my conscious experience is not logically entailed by the facts about my functional organization«. Chalmers, David J.: The conscious mind. In search of a fundamental theory, New York: Oxford University Press 1996, S. 97.

115 Locke hat auf verschiedene Ideen, die im Geist verschiedener Menschen durch dasselbe Objekt erzeugt werden, hingewiesen: »Nehmen wir zum Beispiel an, die Idee, die ein Veilchen im Geist des einen Menschen vermittels der Augen erzeugt, sei dieselbe, die im Geist eines anderen durch die Ringelblume erzeugt werde und umgekehrt «. Locke, John / [Hrsg.: Brandt, Reinhard]: Versuch über den menschlichen Verstand. Band I: Buch I und II, Hamburg: Meiner [zuerst 169o, engl.]/2006, S. 49o. 
Menschen mit invertierten Qualia ausgestattet sein könnten. ${ }^{116}$ Weitere Varianten des modalen Argumentes, auf die ich im Rahmen dieser Arbeit jedoch nicht näher eingehen kann, sind das Teil-Zombie-Argument und Argumente mit »schwindenden« oder »tanzenden « Qualia. ${ }^{117}$ Modale Argumente werden also in den verschiedensten Versionen präsentiert. Der Kerngedanke dieses Argumenttyps besteht immer darin, dass in funktionaler Hinsicht identische Systeme divergente (oder zuweilen gar keine) phänomenale Eigenschaften haben könnten. ${ }^{118}$

Modale Argumente können den Anschein erwecken, dass es sich bei ihnen nur um müssige metaphysische Spekulationen darüber handelt, was alles möglich sein könnte. Allerdings hat das modale Argument insofern eine entscheidende Relevanz, als ein wichtiger Aspekt unserer Vorstellungen von

116 Konkret heisst das, dass zwei Menschen, auch wenn sie in neurologischer Hinsicht genau gleich funktionierten, in phänomenaler Hinsicht jeweils entsprechend der anderen Komplementärfarbe (d.h. invertiert) wahrnehmen könnten: diesselben Objekte, die Max als >grün< erlebt, würde Moritz als >rot< wahrnehmen. Da alle Personen, jedoch Farbprädikate auf dieselbe Weise erlernen, würden Max und Moritz das Adjektiv >rot gleich verwenden. Beide würden also im Urteil übereinstimmen, dass Tomaten rot sind. Max und Moritz würden folglich auch im Hinblick auf ihr Sprachverhalten identisch funktionieren, was zur Folge hat, dass ihre Qualia-Inversion von aussen bzw. von einer Dritten-PersonPerspektive nicht feststellbar ist. Dennoch würde Max, der alle Farben invertiert erlebt, Tomaten phänomenal als etwas `Grünes< erleben - d.h. so wie Moritz, der Normalsichtige, die Farbe grün erlebt. Eine Ausarbeitung des Qualia-Inversions-Argument findet sich bei Ned Block, der davon ausgeht, dass die Möglichkeit einer Qualia-Inversion ein Argument gegen den Funktionalismus darstellt: »[...] if two different mental states can play exactly the same functional role, then there is an aspect of mentality (the >qualitative aspect) that eludes characterization in terms of functional role«. Block, Ned J.: »Inverted Earth«, in: Philosophy of mind. Critical concepts in philosophy. Volume 4: Consciousness, Crawford, Sean [Hrsg.], London: Routledge \& Kegan Paul [zuerst 199o]/2011, S. 121. Vgl. dazu auch Walter, Sven: »Allgemeine Einleitung: Phänomenales Bewusstsein: Unlösbares Mysterium oder seriöses wissenschaftliches Problem?«, in: Qualia. Ausgewählte Beiträge, Heckmann, Heinz-Dieter / Walter, Sven [Hrsg.], Paderborn: Mentis 20o1, S. 47. Vgl. ebenfalls Chalmers, David J.: The conscious mind. In search of a fundamental theory, New York: Oxford University Press 1996, S. 263-266.

117 Näheres dazu vgl. Chalmers, David J.: »Bewusstsein und sein Platz in der Natur«, in: Grundkurs Philosophie des Geistes. Band 1: Phänomenales Bewusstsein, Metzinger, Thomas [Hrsg.], Paderborn: Mentis [zuerst 2002, engl.]/2007, S. 124-125. Vgl. ebenfalls Chalmers, David J.: The conscious mind. In search of a fundamental theory, New York: Oxford University Press 1996, S. 247-275.

118 Auf der Denkbarkeit, dass bei identischer Funktionalität auch keine phänomenalen Eigenschaften logisch möglich wären, basieren die modalen Argumente bzw. Gedankenexperimente von Zombies, Teil-Zombies oder sschwindenden< Qualia. Wohingegen Qualia-Inversionen oder >tanzenden` Qualia auf der Denkbarkeit der logischen Möglichkeit divergenter phänomenaler Eigenschaften bei gleicher Funktionalität basiert. 
Kausalität auf kontrafaktischen Analysen aufbaut. ${ }^{119}$ Denn unsere Konzeption darüber, was Ursachen sind, impliziert, dass sie nicht nur notwendig sind, um eine Wirkung zu erzeugen, sondern dass die Ursache ihre Wirkung gleichsam »erzwingen $«^{120}$ muss: das heisst, eine Ursache muss für eine Wirkung auch hinreichend sein. Es genügt nicht, festzustellen, dass bewusstes Sehen immer dann ${ }^{121}$ hervorgebracht wird, wenn Pyramidenzellen in den unteren Schichten des assoziativen visuellen Cortexes (die in rückläufiger Beziehung zum Thalamus stehen) feuern. ${ }^{122}$

Nach Chalmers sind solche Argumente logischer Möglichkeit fundamentale antimaterialistische Argumente. ${ }^{123}$ So ist nach ihm die Denkbarkeit von Zombies ein Argument dafür, dass Bewusstsein nicht logisch über physikalischen Vorgängen superveniert. ${ }^{124}$

119 Vgl. Staudacher, Alexander: Phänomenales Bewusstsein als Problem für den Materialismus, Berlin: De Gruyter 2002, S. 431. Staudacher wendet jedoch ein, dass auch kontrafaktische Abhängigkeitsverhältnisse nicht hinreichend sind, um genuine Ursachen von Epiphänomenen zu unterscheiden. A.a.O., S. 429-431.

120 A.a.O., S. 428.

121 Mit folgendem Beispiel lässt sicht vielleicht noch deutlicher veranschaulichen, dass die Konzeption einer Ursache nicht nur auf einer Wenn-Dann-Regularität beruhen darf: Es wäre falsch, wenn Aliens auf dem Mond aus der Beobachtung, dass immer dann, wenn ein Erdkontinent durch die Erdrotation in die Schattenseite des Sonnenlichtes gelangt, ganz eigentümliche Lichter auf diesem Kontinenten erscheinen, zu folgender Feststellung gelangen: diese Erdlichter werden von der Dunkelheit verursacht, vielleicht aufgrund einer photochemischen Reaktion in Minikratern, die sonst durch Licht blockiert wird und folglich durch die Abwesenheit von Licht verursacht wird. Die Bedingung der Dunkelheit auf der erdabgewandten Seite ist nicht hinreichend für die Lichter auf der Erde, das bedeutet, dass Kausalität nicht nur auf einer Regularität beruht. Genau hier setzen modale Argumente ein: bestimmte physikalische Vorgänge müssen notwendig und hinreichend sein, um bewusstes Erleben hervorzurufen. Das heisst, sie müssen zwinged Erleben verursachen. Genau dies wird auf der Grundlage von modalen Argumenten bezweifelt.

122 Vgl. Crick, Francis / Koch, Christof: »Are we aware of neural activity in primary visual cortex?«, Nature, [1995] Vol. 375, No. 6527, S. 121-123. Vgl. dazu auch Roth, Gerhard: Das Gehirn und seine Wirklichkeit. Kognitive Neurobiologie und ihre philosophischen Konsequenzen, Frankfurt am Main: Suhrkamp 1994, S. 288.

123 Vgl. Chalmers, David J.: The conscious mind. In search of a fundamental theory, New York: Oxford University Press 1996, S. 140.

124 Vgl. a.a.O., S. 93-94. Nach Chalmers ist es jedoch wichtig, zu sehen, dass die Denkbarkeit von Zombies zwar aufzeigt, dass Bewusstsein nicht logisch über physikalischen Vorgängen superveniert, dies aber auch nicht zeige, dass physikalische Zustände und Bewusstsein nicht identisch sein könnten. Vgl. dazu Chalmers, David J.: »Bewusstsein und sein Platz in der Natur«, in: Grundkurs Philosophie des Geistes. Band 1: Phänomenales Bewusstsein, Metzinger, Thomas [Hrsg.], Paderborn: Mentis [zuerst 2002, engl.]/2007, S. 130 . 
If a physically identical zombie world is logically possible, it follows that the presence of consciousness is an extra fact about our world, not guaranteed by the physical facts alone. The character of our world is not exhausted by the character supplied by the physical facts; there is extra character due to the presence of consciousness. ${ }^{125}$

Wichtig ist aber, dass modale Argumente, die auf Supervenienz ${ }^{126}$ bezogen sind, von modalen Argumenten mit Bezug zu Identitätsfragen unterschieden werden. ${ }^{127}$ Modale Argumente mit Identitätsbezug versuchen demnach die Nicht-Identität von Bewusstsein und physikalischen Prozessen aufzuzeigen. Chalmers modale Argumente mit Supervenienzbezug sind demgegenüber ontologisch bescheidener: »The form of the argument is rather, >One can imagine all the physical facts holding without the facts about consciousness holding, so the physical facts do not exhaust all the facts. «128

Die Denkbarkeit von Zombies und von invertierten Qualia sind insofern antimaterialistische Argumente, als sie auf der logischen Möglichkeit eines mit unsererWelt physikalisch identischen Universums mit anderen phänomenalen Tatsachen aufbauen: »As long as some positive fact about experience in our world does not hold in a physically identical world, then consciousness does

125 A.a.O., S. 123 [Hervorhebung im Original].

126 Die Konzeption der Supervenienz geht ganz allgemein davon aus, dass eine bestimmte Formation von (niederstufigen) Eigenschaften eine andere Formation von höherstufigen Eigenschaften bestimmt. Beispielsweise determinieren physikalische Tatsachen biologische Tatsachen, was bedeutet, dass biologische Tatsachen über physikalischen Tatsachen supervenieren. Der Supervenienz-Begriff erlaubt eine einheitliche Terminologie mit der über solche Abhängigkeitsbegriffe diskutiert werden kann, ohne dass dafür den Phänomenen nicht gerecht werdende Identitätsbegriffe verwendet werden müssen. Denn mentale Zustände können einerseits multirealisierbar sein, andererseits scheint zwar das Mentale vom Physischen abhängig zu sein, jedoch nicht unbedingt umgekehrt. Ein anderes Beispiel für den Vorteil des Supervenienz-Begriffs ist der Umstand, dass biologische oder soziale Phänomene (emergente) Eigenschaften besitzen können, die nicht in identischer Weise auf der (sie determinierenden) physikalisch niederstufigen Ebene zu finden sind. Mehr zum Supervenienz-Begriff vgl. Chalmers, David J.: The conscious mind. In search of a fundamental theory, New York: Oxford University Press 1996, S. 32-33. Vgl. dazu auch Staudacher, Alexander: Phänomenales Bewusstsein als Problem für den Materialismus, Berlin: De Gruyter 2002, S. 387.

127 Modale Argumente die sich auf Identitätsfragen beziehen, findet Chalmers auch weniger schlüssig als solche mit Supervenienzbezug. Vgl. Chalmers, David J.: »Bewusstsein und sein Platz in der Natur«, in: Grundkurs Philosophie des Geistes. Band 1: Phänomenales Bewusstsein, Metzinger, Thomas [Hrsg.], Paderborn: Mentis [zuerst 2002, engl.]/2007, S. 131 .

128 Chalmers, David J.: The conscious mind. In search of a fundamental theory, New York: Oxford University Press 1996, S. 131. 
not logically supervene «. ${ }^{129}$ Und wenn Bewusstsein nicht über Physikalischem logisch superveniert, dann ist, Chalmers zufolge, der Materialismus im Sinne einer strikt monistischen Ontologie ${ }^{130}$ falsch.

Nach Chalmers sprechen modale Argumente aber nur für einen nichtreduktiven Funktionalismus. ${ }^{131}$ Denn aufgrund des Prinzips der so genannten »organizational invariance «132 hält er es für extrem unplausibel, dass QualiaInversionen, abwesende, »schwindende « oder »tanzende« Qualia empirisch d.h. nomologisch möglich sind. ${ }^{133}$ Hier ist es wichtig, anzumerken, dass sich nomologische Möglichkeit von der oben erwähnten logischen Möglichkeit unterscheidet. In der Diskussion modaler Argumente gegen den Materialismus sind insgesamt drei Kategorien von Modalitäten feststellbar: ${ }^{134}$

1. logische (begriffliche) Möglichkeit

2. metaphysische (ontologische) Möglichkeit

3. nomologische (natürliche oder empirische) Möglichkeit

1.) Logische Möglichkeit ist gleichbedeutend mit der widerspruchsfreien Denkbarkeit resp. begrifflichen Kohärenz ${ }^{135}$ eines Sachverhaltes. Sie unterscheidet sich jedoch von formallogisch strikter Ableitbarkeit. ${ }^{136}$ Logisch möglich wären auch fliegende Bäume, die in Wolken Flüssigkeit absorbieren.

129 A.a.O., S. 99.

130 So definiert Chalmers diese Form des Materialismus folgendermassen: »[...] I take materialism to be the doctrine that the physical facts about the world exhaust all the facts, in that every positive fact is entailed by the physical facts. If zombie worlds or inverted worlds are possible, the physical facts do not entail all the positive facts about our world, and materialism is false«. A.a.O., S. 123-124. [Hervorhebung E. E.].

131 Chalmers' nicht-reduktiver Funktionalismus geht davon aus, dass funktionale Organisation bewusstes Erleben zwar vollständig determiniert, letzteres aber dennoch nicht auf funktionale Organisation reduzierbar ist. Vgl. dazu a.a.O., S. 274-275.

132 A.a.O., S. 247-251.

133 Vgl. a.a.O., S. 274. Vgl. auch Walter, Sven: ^Allgemeine Einleitung: Phänomenales Bewusstsein: Unlösbares Mysterium oder seriöses wissenschaftliches Problem?«, in: Qualia. Ausgewählte Beiträge, Heckmann, Heinz-Dieter / Walter, Sven [Hrsg.], Paderborn: Mentis 2001, S. $44-56$.

134 Vgl. dazu auch Staudacher, Alexander: Phänomenales Bewusstsein als Problem für den Materialismus, Berlin: De Gruyter 2002, S. 390-391.

135 Begriffliche Kohärenz geht nach Chalmers mit der Vorstellbarkeit eines Sachverhaltes einher. Vgl. Chalmers, David J.: The conscious mind. In search of a fundamental theory, New York: Oxford University Press 1996, S. 35 .

136 Logische Möglichkeit ist deshalb eher als `breite〈logische Möglichkeit zu verstehen:»[...] one can think of it [logische Möglichkeit] loosely as possibility in the broadest sense, corresponding roughly to conceivability, quite unconstrained by the laws of our world. [...] This sort of possibility is often called >broadly logicak possibility [...] as opposed to the >strictly logical< possibility that depends on formal systems«. Ebd. 
2.) Ein engerer Spielraum als derjenige der logischen Modalität liegt der metaphysischen Möglichkeit zugrunde. Sie ist enger, weil es bei ihr nicht nur um den logisch möglichen Spielraum unserer Begriffe geht, sondern um das, was in einer möglichen Welt »wirklich «137 der Fall sein könnte. Staudacher verweist diesbezüglich auf den Unterschied zwischen apriorischer und aposteriorischer Notwendigkeit der oft mit dem Unterschied zwischen logischer und metaphysischer Modalität verglichen wird. ${ }^{138}$ Nach Chalmers ist hingegen metaphysische Möglichkeit letztlich dasselbe wie logische Möglichkeit, weil (trotz des Einwandes aposteriorischer Notwendigkeit) ${ }^{139}$ jede logisch mögliche Welt auch eine metaphysisch (bzw. ontologisch) mögliche Welt sein könnte. ${ }^{140}$

137 Staudacher, Alexander: Phänomenales Bewusstsein als Problem für den Materialismus, Berlin: De Gruyter 2002, S. 399. Diese Formulierung wirkt etwas widersprüchlich. Staudacher will damit offenbar darauf hinweisen, dass es bei metaphysischer Möglichkeit um den (nicht unbegrenzten) Spielraum geht, was metaphysisch »wirklich« möglich sei. Vgl. ebd. Dieser Spielraum darf jedoch nicht mit nomologischer bzw. natürlicher Möglichkeiten verwechselt werden. Vgl. dazu weiter unten.

138 Es kollidiert bspw. nicht mit apriorischer Notwendigkeit zu denken, dass Wasser $\mathrm{H}_{8} \mathrm{O}$ ist, d.h. diese Phantasie bildet keinen Widerspruch in sich. A priori betrachtet, könnten die Elemente Wasserstoff und Sauerstoff auch andere Bindungseigenschaften haben. Dennoch sei es eine aposteriorische Notwendigkeit, dass eine Substanz, die genau wie Wasser ist, in jeder Welt $\mathrm{H}_{2} \mathrm{O}$ sein muss, weil der Ausdruck >Wasser nicht nur in dieser Welt, sondern in allen möglichen Welten, auf dieselbe Entität referiert und somit als >starrer Designator zu verstehen ist. Aus diesem Grund sei es auch in anderen möglichen Welten unmöglich, dass Wasser etwas anderes als $\mathrm{H}_{2} \mathrm{O}$ sein könne. Vgl. dazu auch a.a.O., S. 398. Vgl. ebenfalls Kripke, Saul A.: Naming and necessity, Oxford: Blackwell [zuerst 1972]/1980, S. 97-105. Vgl. auch die nächste Fussnote.

139 Der Einwand aposteriorischer Notwendigkeit wird oft im Zusammenhang mit dem Unterschied zwischen Denkbarkeit und wahrer Möglichkeit angewendet: Die Existenz der so genannten >notwendigen Wahrheit‘, dass Wasser identisch mit $\mathrm{H}_{2} \mathrm{O}$ ist, könne nur $a$ posteriori gewusst werden (im Gegensatz zu apriorisch logischer Möglichkeit). Demnach sei es zwar logisch möglich, dass Wasser nicht $\mathrm{H}_{2} \mathrm{O}$ sein könnte, aber ontologisch sei dies nicht (bzw. in keiner Welt) möglich. Chalmers, David J.: The conscious mind. In search of a fundamental theory, New York: Oxford University Press 1996, S. 131.

140 Vgl. a.a.O., S. 134. Vgl. dazu auch Staudacher, Alexander: Phänomenales Bewusstsein als Problem für den Materialismus, Berlin: De Gruyter 2002, S. 397. Innerhalb dieser Arbeit ist es nicht möglich, auf die semantischen Finessen dieser zwei modalen Unterscheidungsgrade einzugehen. Im Hinblick auf die Einwände, die sich auf aposteriorische Notwendigkeit beziehen, und damit gegen die Gleichsetzung von logischer und metaphysischer Möglichkeit argumentieren, argumentiert Chalmers in einer ziemlich komplizierten Diskussion über primäre Intensionen in der aktuellen Welt und sekundäre Intensionen in kontrafaktischen Welten dafür, dass aposteriorische Notwendigkeit im Hinblick auf Bewusstsein irrelevant ist, weil der Unterschied von primärer und sekundärer Intension bei Bewusstsein zusammenfällt: damit etwas ein bewusstes Erlebnis in der aktuellen Welt ist, muss es sich lediglich phänomenal >anfühlen < - Chalmers nennt diese Eigenschaft "phenomenal feel«. Chalmers, David J.: The conscious mind. In search of a fundamental 
3.) Nomologisch bzw. empirisch möglich ist dagegen alles, was unter Berücksichtigung der physikalischen Gesetze möglich ist. Dieser Möglichkeitsrahmen gilt als noch enger, als der Rahmen des metaphysisch Möglichen. ${ }^{141}$ Nomologische Möglichkeit stellt folglich auch ein engerer Möglichkeitsrahmen dar, als die logische Modalität: Auch wenn man sich auf kohärente und widerspruchsfreie Weise ein Universum denken kann, in dem Antigravitation vorherrscht (logische Modalität), so ist es nomologisch nicht möglich, dass in unserer Welt überreife Äpfel gegen den Himmel fliegen.

Aus den drei erwähnten Modalitätsformen folgen zwei ${ }^{142}$ verschiedene Supervenienzthesen. A.) Nomologische bzw. natürliche Supervenienz besteht dann, wenn phänomenale Eigenschaften naturgesetzlich über physikalischen Eigenschaften supervenieren. Falls nomologische Supervenienz besteht, dann muss es bestimmte Naturgesetze geben, die garantieren, dass wenn eine bestimmte physikalische Eigenschaft vorliegt, zugleich auch Phänomenales instantiiert sein muss. ${ }^{143}$ Auch wenn letzteres vermutlich der Fall ist, so lässt sich eine Supervenienzbasis neurophysiologischer Vorgänge auch kohärent ohne Vorliegen der supervenienten Eigenschaft 〉Bewusstsein< denken. ${ }^{144}$ Ein

theory, New York: Oxford University Press 1996, S. 133. Gleichermassen muss auch in einer kontrafaktischen Welt etwas, um ein bewusstes Erlebnis zu sein, nur diese Eigenschaft haben: »All it means to be a conscious experience, in any possible world, is to have a certain feel«. Ebd.

Mit dieser Gleichsetzung von primärer und sekundärer Intension von Bewusstsein will Chalmers begründen, dass aposteriorische Notwendigkeit im Hinblick auf Bewusstsein irrelevant ist: der Unterschied zwischen blossem »watery stuff« einer kontrafaktischen Welt und echtem Wasser auf unserer Welt, auf welchem aposteriorische Einwände gegen Modale Argumente basieren, ist bei Bewusstsein nicht vorhanden: »[...] if something feels like a conscious experience, even in some counterfactual world, it is a conscious experience «. Ebd. [Hervorhebung im Original]. Am Ende dieser Überlegungen gelangt er dann zum Fazit, dass diese semantischen Differenzen letztlich nicht von fundamentaler Bedeutung für das hard problem seien: »We can simply forget the semantics of these terms, and note that the relevant possible world clearly lacks something, whether or not we call it >consciousness««. A.a.O., S. 134 [Hervorhebung im Original].

141 Es ist metaphysisch möglich, dass es Welten mit anderen Naturgesetzen gibt. Insofern gibt es mehr Situationen, die metaphysisch möglich sind, als solche die nomologisch oder natürlich möglich sind. Vgl. Staudacher, Alexander: Phänomenales Bewusstsein als Problem für den Materialismus, Berlin: De Gruyter 2002, S. 399.

142 Weil nach Chalmers metaphysische Möglichkeit letztlich dasselbe wie logische Möglichkeit ist, erörtert er nur eine logische und eine nomologischen Supervenienz. Vgl. Chalmers, David J.: The conscious mind. In search of a fundamental theory, New York: Oxford University Press 1996, S. 131.

143 Vgl. Staudacher, Alexander: Phänomenales Bewusstsein als Problem für den Materialismus, Berlin: De Gruyter 2002, S. 391.

144 Vgl. a.a.O., S. 423. 
strikt monistischer Materialismus kann sich aber nicht bloss auf nomologischer Supervenienz abstützen, denn die nomologische Folgebeziehung zwischen neurophysiologischen Vorgängen und bewusstem Erleben wirkt kontingent. ${ }^{145}$

B.) Logische Supervenienz hängt deshalb mit Ableitbarkeit zusammen, weil sie überall dort vorliegt, wo B-Eigenschaften mit A-Eigenschaften reduktiv erklärt werden können. Wenn die B-Eigenschaft (z.B. Wasser) logisch über der A-Eigenschaften $\left(\mathrm{H}_{2} \mathrm{O}\right)$ superveniert, dann gibt es keine zwei logisch möglichen Situationen, in welchen $\mathrm{H}_{2} \mathrm{O}$ vorliegt ohne, dass Wasser vorliegt. Logische Supervenienz bedeutet in diesem Fall, dass es nicht einmal vorstellbar ist, dass irgendwo eine Ansammlung von $\mathrm{H}_{2} \mathrm{O}$-Molekülen ist, ohne dass auch Wasser vorhanden ist. ${ }^{146}$ Die Relevanz logischer Supervenienz besteht nun darin, dass mit ihr immer auch eine »lückenlose reduktive Erklärung «147 vorliegt. Denn bei ihr superveniert die Eigenschaft, die das Explanandum darstellt (z.B. Wasser) logisch zwingend über der Eigenschaft, die das Explanans (z.B. $\mathrm{H}_{2} \mathrm{O}$ ) darstellt. Es ist diese logisch zwingende Supervenienz des Explanandums über dem Explanans, welche eine lückenlose Erklärung garantiert.

Wenn bewusstes Erleben jedoch nicht zwingend über physiologischen Eigenschaften superveniert, könnten identische physiologische Eigenschaften, die angeblich das Explanans darstellen, vorliegen, ohne dass phänomenale Eigenschaften darüber supervenieren. Dies hat zur Folge, dass die Eigenschaft >Bewusstsein<, die das Explanandum darstellt, nicht lückenlos mit physiologischen Vorgängen erklärt werden kann. Ein materialistisches Gegenargument besteht nun darin, festzustellen, dass logische Supervenienz noch keine metaphysische Supervenienz und schon gar keine nomologische Supervenienz impliziere. ${ }^{148}$ Aus diesem Grund sei nicht bewiesen, dass es

145 Vgl. Chalmers, David J.: The conscious mind. In search of a fundamental theory, New York: Oxford University Press 1996, S. 41. Das heisst, selbst wenn eine nomologische Supervenienz von neurophysiologischen Vorgängen und bewusstem Erleben besteht, so schliesst dies nicht die logische Möglichkeit aus, dass in zwei physikalisch identischen Welten Phänomenales divergent supervenieren könnte. Vgl. Staudacher, Alexander: Phänomenales Bewusstsein als Problem für den Materialismus, Berlin: De Gruyter 2002, S. 392.

146 In solch einem Fall muss logisch zwingend Wasser vorhanden sein, wenn $\mathrm{H}_{2} \mathrm{O}$ vorhanden ist. Anders gesagt: zwischen der Supervenienzbasis $\mathrm{H}_{2} \mathrm{O}$ und den darüber supervenierenden Eigenschaften von Wasser besteht eine logische Supervenienz. Vgl. dazu a.a.O., S. 39o.

147 Ebd.

148 Einfach gesagt: Denkbarkeit bedeute noch nicht, dass etwas auch empirisch der Fall sei (nomologische Möglichkeit) oder grundsätzlich der Fall sein könnte (metaphysische Möglichkeit). Vgl. dazu a.a.O., S. 423 . 
auch metaphysisch möglich sei, ${ }^{149}$ dass über zwei Systemen mit identischen physiologischen Eigenschaften divergierende phänomenale Eigenschaften supervenieren könnten. ${ }^{150}$ Folglich wäre auch nicht bewiesen, dass es eine Erklärungslücke gibt.

Nach Chalmers genügt aber bereits das Fehlen von zwingender logischer Supervenienz um festzustellen, dass Bewusstsein nicht reduktiv erklärt werden kann. ${ }^{151}$ Denn Erklärbarkeit gründet auf apriorischen Implikationen bzw. auf logischer Supervenienz phänomenaler über physikalischen Tatsachen. Erklärungen gründen demzufolge auf apriorischen Folgebeziehungen, weil letztere eine fundamentale Form epistemischer Folgebeziehungen sind, die erfahrungsunabhängig impliziert werden. ${ }^{152}$ Aus diesem Grund sind auch Einwände, die mit der Unterscheidung von apriorischer und aposteriorischer Notwendigkeit die Vergleichbarkeit von logischer und metaphysischer Supervenienz kritisieren, letztlich nicht relevant. ${ }^{153}$ Daraus kann wiederum gefolgert werden, dass aufgrund der fehlenden logischen Notwendigkeit, dass Phänomenales über Physikalischem superveniert, auch keine ontologische Supervenienz vorhanden ist. Ein ontologisch reduktiver Materialismus wäre also nur dann wahr, wenn unter allen metaphysischen Umständen das Konditional $\mathrm{P} \rightarrow \mathrm{Q}$ (»wenn $\mathrm{P}$ dann $\mathrm{Q} \ll)^{154}$ zwingend zutreffen würde bzw. P erzwingt $Q$ unter allen denkbaren metaphysischen Umständen.

Der Materialismus müsste deshalb darlegen, dass logische Supervenienz von Phänomenalem über Physikalischem zwingend ist, weil eine befriedigende reduktive Erklärung von Bewusstsein auf apriorisch zwingenden Implikationen gründen muss. ${ }^{155}$ Mit anderen Worten: Bewusstsein ist nur dann reduktiv erklärbar, wenn es auf physikalischen Eigenschaften logisch superveniert:

\footnotetext{
149 Das heisst, dass logische Möglichkeit noch nicht beweise, dass etwas metaphysisch oder gar empirisch der Fall sei.

150 Vgl. Chalmers, David J.: The conscious mind. In search of a fundamental theory, New York: Oxford University Press 1996, S. 98.

151 Vgl. ebd.

$15^{2}$ Vgl. dazu ebd. Vgl. dazu auch Chalmers, David J.: »Bewusstsein und sein Platz in der Natur«, in: Grundkurs Philosophie des Geistes. Band 1: Phänomenales Bewusstsein, Metzinger, Thomas [Hrsg.], Paderborn: Mentis [zuerst 2002, engl.]/2007, S. 127.

153 Vgl. Chalmers, David J.: The conscious mind. In search of a fundamental theory, New York: Oxford University Press 1996, S. 98.

154 Wobei $P$ für eine physikalische Eigenschaft und Q für eine phänomenale Eigenschaft steht.

155 Vgl. ebd.
} 
A natural phenomenon is reductively explainable in terms of some low-level properties precisely when it is logically supervenient on those properties. It is reductively explainable in terms of physical properties - or simply >reductively explainable $<-$ when it is logically supervenient on the physical. ${ }^{156}$

Somit wäre Chalmers zufolge ein reduktiver Materialismus nur dann wahr, wenn er auf logisch zwingenden Supervenienzbeziehungen zwischen Physikalischem und Phänomenalem gründet. ${ }^{157}$ Gerade dies wird jedoch mit Hilfe der in diesem Abschnitt erörterten modalen Argumente bezweifelt. ${ }^{158}$

\subsection{Problematische Umgangsweisen mit dem hard problem}

Im Zusammenhang mit meiner Erörterung des Forschungsstandes erwähne ich im folgenden Abschnitt bestimmte Aspekte der Diskussion um das hard problem, die vernachlässigt werden. Andererseits kritisiere ich bestimmte Argumentationsmuster, die relativ unhinterfragt und dennoch fast inflationär angewendet werden. Dieser Teil der Kritik bezieht sich auch auf problematische Strategien und beweislastabschiebende Diskursformen von Seiten der Reduktionisten.

\subsubsection{Reduktionismus als dogmatische Default-Position mit unklarem Physikalismus-Begriff}

Bei der Durchsicht des philosophischen Diskurses über Qualia und das hard problem fällt auf, dass ziemlich optimistische Intuitionen über reduktivphysikalistische Konzeptionen bewussten Erlebens, entgegen aller Schwierigkeiten weit verbreitet sind. ${ }^{159}$ Diese Defaultposition wird mit einer - insbesondere für einen philosophischen Diskurs - oft überraschenden und

156 A.a.O., S. $47-48$.

157 Vgl. a.a.O., S. 41. Vgl. dazu auch Staudacher, Alexander: Phänomenales Bewusstsein als Problem für den Materialismus, Berlin: De Gruyter 2002, S. 394.

${ }_{15} 8 \mathrm{Zu}$ modalen Argumenten vgl. auch Heckmann, Heinz-Dieter: »Qualia-basierte Argumente gegen den Materialismus«, in: Bewusstsein und Repräsentation, Esken, Frank / Heckmann, Heinz-Dieter [Hrsg.], Paderborn: Mentis 1999, S. 337-351. Vgl. ebenfalls Block, Ned J.: »Are Absent Qualia Impossible?«, Philosophical Review, [1980] Vol. 89, No. 2.

159 Zur Dominanz von naturalistischen Positionen in der Philosophie des Geistes vgl. Crane, Tim: »The origins of qualia«, 20oo, Online: http://sas-space.sas.ac.uk/22o/1/The\%2o origins\%20of\%2oqualia.pdf [zuletzt geprüft am 24.09.2018], S. 3 . 
zuweilen kaum hinterfragten Selbstverständlichkeit ${ }^{160}$ oder sogar apodiktischer Manier behauptet. ${ }^{161}$ Demgemäss konstatiert Schildknecht, dass in der Philosophie des Geistes eine beträchtliche Tendenz mit reduktionistischer Voreingenommenheit vorhanden ist: »The route that philosophers of mind opt [...] displays a considerable bias towards reductionistic conceptions of phenomenal consciousness «. ${ }^{162}$ Leopold Stubenberg stellt bezüglich dieser Tendenz sogar eine »ideologische Komponente «(»ideological component «) $)^{163}$ fest. Freilich finden sich auch Positionen, die weniger voreingenommen sind oder einen Mittelweg zu gehen versuchen. ${ }^{64}$ Besonders apodiktisch ist hingegen bspw. Papineau. Er scheint davon auszugehen, dass das hard problem auf die einfache Frage reduzierbar sei, warum »nicht akzeptiert« werden könne,

16o So spricht Stubenberg von einem unhinterfragten Bekennen zu materialistischen Theorien des Geistes, was für ihn zugleich die Ursache unserer Verwirrung über Qualia ist: »Our particular bafflement is rooted in an unquestioned commitment to materialism«. Stubenberg, Leopold: Consciousness and qualia, Amsterdam: John Benjamins 1998, S. 31 [Hervorhebung E. E.].

161 Papineau setzt bspw., aufgrund von Apriori-Überlegungen, die ontologische Identität von phänomenalen und materiellen Eigenschaften einfach voraus, so dass es nach ihm gar kein hard problem gibt: »[...] identities need no explaining«. Papineau, David: »Mind the Gap«, Philosophical Perspectives, [1998] Vol. 12: Language, Mind, and Ontology, S. 379.

Diese Haltung lässt sich nach Demmerling manchmal auch in der Hirnforschung beobachten: »Wissenschaft und Technik können die Gestalt von Ideologie annehmen, zumal dann, wenn sie den Anspruch erheben, das Ganze der Welt und des menschlichen Lebens zu erklären. Man gewinnt in derartigen Fällen gelegentlich den Eindruck, die Vertreter der Wissenschaften seien in die Rolle von Priestern geschlüpft, um das Erbe religiöser und im schlechten Sinne metaphysischer Weltdeutungen anzutreten. Wer während der vergangenen Jahre beispielsweise die Debatten um die Reichweite der Hirnforschung verfolgt hat, konnte in der Tat den Eindruck gewinnen, eine neue Religion sei entstanden, in welcher der Anspruch auf umfassende und letzte Erklärungen des menschlichen Denkens, Redens und Handelns mit weitreichenden Heilsversprechen verbunden wurde«. Demmerling, Christoph: »Schwerpunkt: Kritische Philosophie der Neurowissenschaften«, Deutsche Zeitschrift für Philosophie, [2011] Vol. 59, No. 3, S. 37 O.

162 Schildknecht, Christiane: Sense and self. Perspectives on nonpropositionality, Paderborn: Mentis 2002, S. 196.

163 Stubenberg, Leopold: Consciousness and qualia, Amsterdam: John Benjamins 1998, S. 31.

164 Vgl. dazu Schildknecht, Christiane: Sense and self. Perspectives on nonpropositionality, Paderborn: Mentis 2002, S. 196, Fussnote 717. Diesen Mittelweg versucht bspw. Bieri zu gehen, indem er feststellt, dass die Philosophie des Geistes eine Art »Metatheorie der empirischen Wissenschaften vom Mentalen« sei: »Durch den wissenschaftlichen Realismus wird die Philosophie des Geistes zur Philosophie der empirischen Psychologie«. Demnach besteht eine wichtige Aufgabe der Philosophie des Geistes, die ihr »von den wissenschaftlichen Realisten « zugewiesen wird, darin, eine Vermittlerrolle zwischen »neuen empirischen Theorien « und »unseren alten mentalistischen Beschreibungen« einzunehmen. Vgl. Bieri, Peter [Hrsg.]: Analytische Philosophie des Geistes, Weinheim: Beltz 2007, S. 25 . 
dass die Eigenschaften von Bewusstsein »identisch $\aleph^{165}$ mit »materiellen Eigenschaften $\ll^{166}$ seien:

[...] the problem of explaining the appearance of an explanatory gap reduces to the problem of explaining why most people are so resistant to accepting that conscious properties are identical with material properties. ${ }^{167}$

Ein gutes Beispiel für eine ähnlich dogmatische Manier des Eliminativismus, ist Dennett, der mit Hilfe seiner »Intuitionspumpen « (»intuition pumps«) $)^{168}$ nachzuweisen versucht, dass es keine subjektiven phänomenalen Eigenschaften $»$ gibt $«, 169$ sondern nur $»$ relationale, extrinsische Eigenschaften $\ll, 170$ womit sich seiner Meinung nach die »Verwirrung ${ }{ }^{171}$ im Hinblick auf das hard problem auflöst. ${ }^{172}$

Insbesondere eliminativistische Positionen scheinen anfällig zu sein, sich in Behauptungen zu verlieren, die etwas stark Dogmatisches an sich haben. Auf den Einwand, wie denn überhaupt die Frage nach bewusstem Erleben in die philosophische Reflexion geraten kann, wenn es doch angeblich gar nicht >existiere $<$, wird entgegnet, dass es eben nur so »scheine «, ${ }^{173}$ dass wir erleben auch wenn der Begriff des \Scheinens $<$ doch die Kategorie des Erlebens geradezu voraussetzt. ${ }^{174}$ Diese Ad-hoc-Hypothese, weist jedoch eine ähnlich zirkuläre Argumentationsstruktur auf, mit dem man auch eine solipsistische Ontologie verteidigen könnte. Wenn wir einem Solipsisten mit dem Einwand begegneten,

165 Papineau, David: »Mind the Gap«, Philosophical Perspectives, [1998] Vol. 12: Language, Mind, and Ontology, S. 384 .

166 Ebd.

167 Ebd. [Hervorhebung E.E.].

168 Dennett, Daniel C.: »Quining Qualia«, in: Philosophy of mind. Classical and contemporary readings, Chalmers, David J. [Hrsg.], New York: Oxford University Press [zuerst 1988]/2002, S. 227.

169 A.a.O., S. 244.

170 Ebd.

171 A.a.O., S. 227.

172 Dennett glaubt zwar mit Hilfe seiner »Intuitionspumpen « (»intuition pumps«) nachzuweisen, dass es keine Qualia »gibt«, verwendet innerhalb seiner Argumentation einen besonders engen Qualia-Begriff, der gar nicht phänomenales Erleben in seiner Breite abdeckt. Somit kommt er der Beweislast, dass es kein Erleben >gibt<, leider auch nicht nach. Vgl. dazu a.a.O., S. 227 und 244. Mehr dazu in den Abschnitten 8.1, 9 und 10.1.

173 Vgl. z.B. Dennett, Daniel C.: »Why and How Does Consciousness Seem the Way it Seems?«, 2015, Online: http://open-mind.net/papers/why-and-how-does-consciousness-seem-theway-it-seems [zuletzt geprüft am 24.09.2018], S. 3.

174 Zur dogmatisch anmutenden Behauptung, dass Bewusstsein einfach eine >lllusion< sei, vgl. auch Chalmers, David J.: The conscious mind. In search of a fundamental theory, New York: Oxford University Press 1996, S. xii. 
dass der Einfluss der physischen Substanz Paracetamol auf sein Schmerzerleben darauf hinweist, dass es offensichtlich physische Phänomene gibt, so könnte er - analog zum Eliminativist, aber mit umgekehrten Vorzeichen - die Prämisse aufrecht erhalten, dass Phänomene, die auf Physikalisches hinweisen, nicht wirklich >gibt ${ }^{175}$ Er würde sich - ebenfalls analog zum Eliminativist darauf berufen, dass es eben nur so >scheine<, als ob es eine Aussenwelt gäbe. ${ }^{176}$ Denn, so seine weitere Argumentation, unser eigenes Bewusstsein sei die Ursache für die >Illusion<, dass es etwas geben soll, das >extrinsisch < wirke oder >physikalisch « sei und die, für einen Solipsisten >eigenartige< Eigenschaft der >Objektivität haben soll. Bei weiteren Einwänden zur Implausibilität seiner These könnte ein Solipsist (wieder analog zum Eliminativismus) das Argument bemühen, dass dies einfach so schwer einzusehen sei, weil es unserer dualistischen >Intuition ‘ widerspreche. Und schliesslich käme er zum Schluss, dass Physikalisches einfach keinen >Platz $<$ hat in einer Welt, die letztlich nur aus Bewusstsein besteht. ${ }^{177}$

175 Denn nach dem Solipsismus, der als radikale Form eines erkenntnistheoretischen Idealismus bezeichnet werden kann, existiert alleine das eigene Ich wirklich; andere Subjekte existieren nur in der Vorstellung. Deshalb kann es nach dieser Ontologie auch keine, vom eigenen Bewusstsein unabhängige Aussenwelt geben. Der Solipsismus wurde zwar in der Geschichte der Philosophie kaum je ernsthaft vertreten, deren theoretische Möglichkeit ist jedoch ein zentrales Thema der Philosophie. Vgl. dazu auch Gabriel, Gottfried: "Solipsismus«, in: Enzyklopädie Philosophie und Wissenschaftstheorie. Band 3: P-So, Mittelstrass, Jürgen [Hrsg.], Stuttgart: Metzler 2004, S. 839.

${ }_{176}$ Vgl. dazu Dennett, Daniel C.: »Lovely and Suspect Qualities«, 1991, Online: http:// cogprints.org/263/1/lovely\%26s.htm [zuletzt geprüft am 24.09.2018].

177 Darüber hinaus wären wohl >objektiveく Eigenschaften gar nicht relevant für einen Solipsisten, da es so etwas innerhalb der solipsistischen Ontologie gar nicht >geben kann; und deshalb auch nicht relevant für solipsistische Erklärungen wäre (analog zum Argument, dass Phänomenales irrelevant sei, für die Erklärung physikalischer Prozesse - in einer Ontologie, in der es per se nur >Objektives` geben kann). Eine monistisch-materialistische Analogie dazu findet sich bei Dennett, für den das Problem phänomenaler Bewusstseinszustände nur in (der Dritten-Person-Perspektive zugänglichen) Sprachäusserungen von Personen liegt, die über phänomenale Zustände berichten. Vgl. dazu Dennett, Daniel C.: The intentional stance, Cambridge, Massachusetts: MIT Press 1987. Vielleicht würde ein Solipsist sogar darauf setzten, dass die solipsistische Philosophie ssicher bald <eine Erklärung für dieses Phänomen finden wird und dass es in Zukunft vielleicht sogar >offensichtlich 〈 werde, dass vermeintlich >Physikalisches « durch das eigene Bewusstsein hervorgerufen werde. Analog dazu hofft ja auch Churchland, dass die Phänomene des bewussten Erlebens nur darauf »warten«, dass sie durch eine reduktiv-materialistische Theorie des Geistes bzw. durch eine Theorie des Gehirns ersetzt werden. Vgl. Churchland, Paul M.: »Die Wiederentdeckung des Lichtes«, in: Grundkurs Philosophie des Geistes. Band 1: Phänomenales Bewusstsein, Metzinger, Thomas [Hrsg.], Paderborn: Mentis [zuerst 1996, engl.]/2007, S. 194. Eine Parallele zur Idee der >Offensichtlichkeit findet sich bei Mandik, der darauf hofft, dass es in Zukunft offensichtlich 
Aus diesen Überlegungen lässt sich folglich ableiten, dass die Plausibilität eines radikal monistischen Solipsismus mit der des radikal monistischen Eliminativismus vergleichbar ist; nach McGinn ist ein skeptizistischer Solipsismus sogar noch plausibler als der Eliminativismus. ${ }^{178}$ Auch wenn eine radikal monistische Position wie der Eliminativismus den Vorteil einer einfacheren Ontologie hat, so findet man in ihm keine schlüssigen Gründe für den Materialismus. ${ }^{179}$ Stubenberg stellt im Gegenteil fest, dass selbst harte Materialisten wie z.B. J.J. C. Smart zugeben, dass es sich bei ihrer Verpflichtung zu solchen Positionen letztlich um ein Bekenntnis ${ }^{180}$ handelt. ${ }^{181}$

werden könnte, wie phänomenales Bewusstsein durch Physikalisches hervorgerufen werde. Vgl. Mandik, Pete / Weisberg, Josh: »Type-q materialism«, 20o8, Online: http:// www.petemandik.com/philosophy/papers/typeq.pdf [zuletzt geprüft am 13.o9.2018], S. 7. Man mag hier vielleicht einwenden, dass die Erfolge der Naturwissenschaft doch aufzeigen, dass die Ontologie eines monistischen Materialismus plausibler ist, als diejenige des Solipsismus. Damit würde jedoch die instrumentelle Ebene naturwissenschaftlicher Forschung mit der metaphysischen Ebene ontologischer Prämissen vermengt. Aus den instrumentellen Erfolgen der Naturwissenschaft, die bspw. in Voraussagen von kausalen Ereignisketten bestehen, lassen sich keine Folgerungen ziehen, die etwas auf einer metaphysischen Ebene ontologischer Prämissen zwingend beweisen würden. So könnten die instrumentellen Erfolge der Wissenschaft, die aus dem Verstehen von kausalen $\mathrm{Zu}$ sammenhängen herrühren, ebenfalls von Solipsisten mit dem Argument, dass >letztlich< alles - auch die Wahrnehmung kausaler Vorgänge - im eigenen Bewusstsein existiere, in jene solipsistische Bewusstseins-Ontologie integriert werden.

Vgl. dazu auch die Darstellung von Alan F. Chalmers über die Zweifel an der Position des wissenschaftsphilosophischen Realismus von Seiten wissenschaftsphilosophischer Anti-Realisten, die auch Instrumentalisten genannt werden. Chalmers, Alan F. / [Hrsg.: Bergemann Niels / Altstötter-Gleich Christine]: Wege der Wissenschaft. Einführung in die Wissenschaftstheorie, Berlin: Springer [zuerst 1976, engl.]/2001, S. 179-195. Für antirealistische Wissenschaftstheoretiker sind wissenschaftliche Theorien »[...] nichts anderes als nützliche Instrumente, die uns dabei unterstützen, Ergebnisse aus Beobachtungen und Experimenten in Verbindung zu bringen und vorherzusagen«. A.a.O., S. 185. Demnach lässt sich von einer Theorie gar nicht sagen, ob sie, in einem metaphysischen Sinne, >wahr oder >falsch $<$ ist. Vgl. ebd.

178 »Während es für mich unvorstellbar ist, dass mein Bewusstsein nicht existieren sollte, ist es für mich durchaus vorstellbar, dass das Ihre möglicherweise nicht existent ist:Vielleicht sind Sie nur ein seelenloser Roboter«. McGinn, Colin: Wie kommt der Geist in die Materie? Das Rätsel des Bewusstseins, München: Beck 2001, S. 221 [Hervorhebung von E. E.].

179 Vgl. dazu auch Stubenberg, Leopold: Consciousness and qualia, Amsterdam: John Benjamins 1998, S. 31.

180 In Stubenbergs Worten: »largely a confession of faith «. Ebd.

181 J. J. C. Smart gibt tatsächlich zu, dass es sich bei seinem Bekenntnis zu materialistischen Theorien des Geistes, um etwas handelt, woran er, auch wenn er dafür Gründe sieht, letztlich einfach glaubt: »[...] sensation, states of consciousness, do seem to be the one sort of thing left outside the physicalist picture, and for various reasons I just cannot believe that 
Offenbar hält, wie Staudacher feststellt, die Mehrheit heutiger analytischer Philosophen dennoch den >Materialismus für »unausweichlich «. ${ }^{182}$ Doch die in diesem Zusammenhang verwendeten Begrifflichkeiten eines >Materialismus phänomenalen Tatsachen sind, werden meist nur ungenügend im Hinblick auf ihre Komplexität reflektiert - sie werden öfters einfach implizit mit einem ontologisch-monistischen, reduktiven Materialismus oder gar mit einem »naturwissenschaftlichen Weltbild «183 gleichgesetzt. So meint bspw. Beckermann, dass sich für Eigenschaften wie Qualia »kein Platz in einem naturwissenschaftlichen Weltbild finden $[. ..] \ll^{184}$ liessen. Solche Behauptungen berufen sich jedoch, wie Searle zu Recht kritisiert, nur »irrtümlich «185 darauf, »Wissenschaft «186 zu sein. Denn die Aussage, dass es nur >Objektives« aber nichts Mentales bzw. Subjektives >gibt<, ist eine metaphysische Behauptung und keine wissenschaftliche Feststellung. ${ }^{187}$

Wenn nun Philosophie eine Disziplin ist, deren Tugend in einer kritischen Skepsis gegenüber Denkgewohnheiten besteht - z.B. gegenüber bisherigen Konzeptionen des >Physikalischen $<$ - dann überrascht diese Tendenz zur reduktionistischen Voreingenommenheit umso mehr. Erstens lässt sich mit Staudacher feststellen, dass eine materialistische Theorie des Geistes gar nicht so zwingend ist, wie oft unterstellt wird. ${ }^{188}$ Zweitens lässt sich fragen, ob es denn keine Konzeption des >Physikalischen` geben kann, die >Platz

this can be so«. Smart, John J. C.: »Sensations and Brain Processes«, Philosophical Review, [1959] Vol. 68, No. 2, S. 142 [Hervorhebung E. E.].

182 Staudacher, Alexander: Phänomenales Bewusstsein als Problem für den Materialismus, Berlin: De Gruyter 2002, S. 425. Staudacher begründet diese »Unausweichlichkeit« mit dem Prinzip der kausalen Geschlossenheit der Welt. Hier müsste man beachten, dass es auch nicht-reduktionistische Konzeptionen von Bewusstsein gibt, die nicht dem Prinzip der kausalen Geschlossenheit widersprechen, wie z.B. der neutrale Monismus von Russell, Chalmers nicht-reduktiver Funktionalismus oder der Panpsychismus (mehr dazu in den Abschnitten 10.3 und 10.4).

183 Beckermann, Ansgar: Analytische Einführung in die Philosophie des Geistes, Berlin: W. de Gruyter 2008, S. 456.

184 Ebd. Der Grund für solche Behauptungen liegt wohl in der Befürchtung, dass wenn man nicht voraussetzt, dass Bewusstsein vollständig auf eine materiell-objektive Realität reduzierbar ist, man angeblich ein »wissenschaftliches Gesamtweltbild « preisgibt. Zu diesem Kritikpunkt vgl. Searle, John R. / [Übers.: Gavagai, Harvey]: Die Wiederentdeckung des Geistes, München: Artemis-Verlag \& Winkler [zuerst 1992, engl.]/1993, S. 135. Zur Kritik der damit verbundenen »objektivistischen Ontologien« vgl auch a.a.O., S. 26.

185 A.a.O., S. 24.

186 Ebd.

187 Vgl. dazu auch a.a.O., S. 119 .

188 Vgl. dazu Staudacher, Alexander: Phänomenales Bewusstsein als Problem für den Materialismus, Berlin: De Gruyter 2002, S. 448. 
für phänomenale Eigenschaften hat. ${ }^{189}$ Nach Levine besteht die Grundthese des Physikalismus in der Feststellung, dass alle physikalischen Tatsachen alle metaphysischen, d.h. alle Tatsachen bestimmen. ${ }^{190}$ So einfach, wie sich dies prima facie anhören mag, ist dies jedoch nicht: »It is of course a notoriously difficult matter to specify precisely what this means $[. ..] \ll{ }^{191}$ Umso mehr mag es verwundern, dass innerhalb der Hard-Problem-Kontroverse zwischen so genannten >Materialisten $<$ und >Antimaterialisten<, eine ausführliche Debatte zur Klärung der Begriffe >Materialismus< bzw. >Physikalismus< fehlt. ${ }^{192}$ Trotz der wachsenden Literatur zum hard problem und zur Erklärungslücke glauben die meisten Philosophen »genügend klar ${ }^{193}$ zu wissen, was »Physikalismus « bedeutet: »[...] despite the growing literature on this controversy, most philosophers have a clear enough idea of what physicalism means that they take a stand pro or con regarding it«.194 Analog zur erwähnten Tendenz, dass reduktionistische Ansätze eher mit einem engen Qualia-Begriff operieren, könnte denn auch die implizite Voraussetzung eines konservativen, evtl. zu engen Physik-Begriffs die Grundlage für die Annahme sein, dass es kein >Platz< in einem naturwissenschaftlichen Weltbild für das Phänomenale gibt: »[...] in arguing that consciousness is not entailed by the physics of our world, we have been tacitly assuming that the physics of our world is something like physics as we understand it today $[. ..] \ll .{ }^{195}$

Doch wenn dieser konservative Physik-Begriff überholt würde, dann könnte eben doch `Platz für Bewusstsein und phänomenales Erleben vorhanden sein: $»[\ldots]$ there might be a new kind of physical theory from which consciousness falls out as a consequence $\ll .196$

189 Mehr zu solchen Konzeptionen des Physikalischen, vgl. Abschnitt 10.3.2

190 So konstatiert er: »Whatever happens in the world is ultimately a matter of how certain physical objects and properties are distributed in space-time«. Levine, Joseph: »The Explanatory Gap«, in: The Oxford Handbook of Philosophy of Mind, McLaughlin, Brian P. I Beckermann, Ansgar / Walter, Sven [Hrsg.], Oxford: Oxford University Press 2009, S. 281.

191 Ebd.

192 Eine nennenswerte Ausnahme bildet hier das 243 Seiten umfassende Werk Basic structures of reality. Essays in meta-physics von Colin McGinn. McGinn, Colin: Basic structures of reality. Essays in meta-physics, New York: Oxford University Press 2011.

193 Levine, Joseph: »The Explanatory Gap«, in: The Oxford Handbook of Philosophy of Mind, McLaughlin, Brian P. / Beckermann, Ansgar / Walter, Sven [Hrsg.], Oxford: Oxford University Press 2009, S. 281.

194 Ebd.

195 Chalmers, David J.: The conscious mind. In search of a fundamental theory, New York: Oxford University Press 1996, S. 118 [Hervorhebung E. E.].

196 Ebd. Vgl. dazu auch McGinn, Colin: Basic structures of reality. Essays in meta-physics, New York: Oxford University Press 2011, S. 2o. Vgl. ebenfalls Stubenberg, Leopold:Consciousness and qualia, Amsterdam: John Benjamins 1998, S. 296, 298 und 307. 


\subsubsection{Ungenügende Differenzierung zwischen easy und hard problem}

David Chalmers unterscheidet zwischen "psychologischen «197 und "phänomenalen Eigenschaften «198 des Mentalen, die das Körper-Geist-Problem in zwei grundsätzlich verschiedene Problembereiche aufgliedern: »[...] an easy part and a hard part «.199 Diese Unterscheidung zwischen dem easy und dem hard problem ist im Diskurs moderner Deutungen des Körper-Geist-Problems von zentraler Bedeutung. Denn sie bildet die Basis, die bestimmt, worin das Explanandum des hard problem besteht.

Mit Elitzur können wir feststellen, dass das hard problem leider oft sehr schlecht ausgewiesen wird: »Often, stating a problem well is half the way to its solution. Equally often, the Mind-Body Problem is ill-stated $\ll .{ }^{200}$ Das heisst, dass Lösungsversuche des hard problem nicht selten auf einer ungenauen Analyse darüber basieren, worin das >harte< Problem überhaupt besteht. Dies hat zur Folge, dass vorschnell `Erklärungen< angeboten werden, die, wenn überhaupt, nur dem easy problem beizukommen vermögen. So kann es passieren, dass aufgrund einer fehlenden heuristischen Basis, die in der Vorarbeit der Unterscheidung zwischen hard und easy problem besteht, vorschnell geglaubt wird, eine `Erklärung \& für das hard problem zu haben. Für eine adäquate Erfassung des hard problem ist es deshalb unabdingbar, dieses genau vom easy problem abzugrenzen:

1. Mit dem easy problem verbindet David Chalmers vor allem technische Probleme der Neuro- und Kognitionswissenschaften und damit verbundene Fragestellungen der Philosophie. ${ }^{201}$ Mit den gegenwärtigen neurophysiologischen Erkenntnissen kann man denn auch, zumindest im Ansatz, diejenigen Phänomene erklären, die Chalmers dem easy problem bzw. dem $»$ psychological mind-body problem $\ll^{202}$ zuordnet. ${ }^{203}$

197 Chalmers, David J.: The conscious mind. In search of a fundamental theory, New York: Oxford University Press 1996, S. 24.

198 Ebd.

199 So konstatiert Chalmers: »The division of mental properties into phenomenal and psychological properties has the effect of dividing the mind-body porblem into two: an easy part and a hard part«. Ebd.

200 Elitzur, Avshalom C.:»Consciousness makes a difference: A reluctant dualist's confession«, 2009, Online: http://philpapers.org/archive/ELICMA.pdf [zuletzt geprüft am 13.o9.2018], S. 4 .

201 Vgl. Chalmers, David J.: The conscious mind. In search of a fundamental theory, New York: Oxford University Press 1996, S. xi-xii und 24.

202 A.a.O., S. 25.

203 So konstatiert Chalmers: $»[\ldots]$ we now have a pretty good idea of how a physical system can have psychological properties $[\ldots] \ll$... Ebd. 
Das »einfache Problem «204 besteht in der Erklärung funktionaler Eigenschaften. ${ }^{205}$ Obwohl die technischen Probleme aufgrund der Komplexität bei solchen Fragestellungen enorm gross sein können, ${ }^{206}$ werfen sie keine grundsätzlichen oder gar metaphysischen Rätsel auf. Für das easy problem gibt es eine klar definierte Herangehensweise, die mindestens im Prinzip einfach ist: »One simply needs to tell a story about the organization of the physical system $[\ldots] \ll .207$

2. Beim hard problem verhält es sich anders: »Here, the mind-body problem is as baffling as it ever was «. ${ }^{208}$ Das hard problem ist der schwerer $\mathrm{zu}$ beantwortende Teil des Körper-Geist-Problems und eine seiner schwersten Fragen lautet: »How could a physical system give rise to conscious experience? ${ }^{209}$ Bei ihm geht es um Fragestellungen, über phänomenales bzw. über bewusstes Erleben. Diese werfen schwierige, evtl. sogar metaphysische Rätsel auf. Auch die beeindruckenden Fortschritte in Physik und Kognitionswissenschaften konnten noch keine nennenswerte Antwort auf die Frage geben, warum kognitive Funktionen von bewusstem Erleben begleitet werden. ${ }^{210}$ Nach Chalmers können wir

204 A.a.O., S. xi. Chalmers spricht diesbezüglich auch vom »leichten Teil« (»easy part«) des »Körper-Geist-Problems« (»mind-body problem«). A.a.O., S. 24.

205 Z.B. die Fähigkeit kognitiver Systeme auf Umweltstimuli zu reagieren und sie zu kategorisieren; kognitive Prozesse der Integration von Information, die Fähigkeit, Erleben verbal auszudrücken; die willkürliche Kontrolle des Verhaltens oder es besteht in Erklärungen wie ein physikalisches bzw. biologisches System lernfähig sein kann bzw. in Fragen zur Messbarkeit der Unterscheidung zwischen Schlaf- und Wachzustand etc. Vgl. a.a.O., S. xi-xii und 24. Vgl. dazu auch Walter, Sven:»Allgemeine Einleitung: Phänomenales Bewusstsein: Unlösbares Mysterium oder seriöses wissenschaftliches Problem?«, in: Qualia. Ausgewählte Beiträge, Heckmann, Heinz-Dieter / Walter, Sven [Hrsg.], Paderborn: Mentis 2001, S. 24-25.

206 Vgl. dazu auch Roth, Gerhard: Das Gehirn und seine Wirklichkeit. Kognitive Neurobiologie und ihre philosophischen Konsequenzen, Frankfurt am Main: Suhrkamp 1994, S. 276-277.

207 Chalmers, David J.: The conscious mind. In search of a fundamental theory, New York: Oxford University Press 1996, S. 24.

208 A.a.O., S. 25 .

209 Ebd.

210 Vgl. ebd. Zudem werfen die zweifellos zahlreichen, immer besser erforschten und nicht minder interessanten Erkenntnisse im Zusammenhang mit dem easy problem wieder Fragen zum hard problem auf. Das lässt sich bspw. im Hinblick auf die oben erwähnte Erkenntnis, dass die verschiedenen Sinnessysteme des Menschen dieselbe \Sprache benutzen, aufzeigen. (Das heisst, die durch einen Reiz ausgelösten Formen des Aktionspotenzials (spikes) sind in den verschiedensten Systemen identisch.) So wirft diese (easy problem-) Erkenntnis gleich wieder Fragen auf, die zum hard problem gehören: (wie) kann der Ort eines Neurons im Gehirn für eine visuelle Erfahrung bestimmend sein, wenn dieses Neuron dasselbe Erregungsmuster aufweist, wie z.B. ein Neuron aus einem 
Fragen zum Zusammenhang von physiologischen Eigenschaften und bewusstem (phänomenalen) Erleben in zwei Teile zerlegen: 1.) Fragen über den Zusammenhang von physiologischen und psychologischen Eigenschaften. 2.) Fragen über das Verhältnis von psychologischen und phänomenalen Eigenschaften. Die Zusammenhänge zwischen physiologischen und psychologischen Eigenschaften können heute im Ansatz, erklärt werden - was aber unbeantwortet bleibt ist das »mind-mind problem «:211 Das heisst, was noch schlecht verstanden wird, ist die Verbindung zwischen psychologischen Eigenschaften, ${ }^{212}$ und phänomenalen Eigenschaften. ${ }^{213}$ Nach Walter ist das hard problem nicht ausschliesslich quantitativer Art, sondern auch qualitativer Art, denn die Frage stellt sich, warum überhaupt qualitativ-subjektive, d.h. phänomenale Gehalte mit quantitativ erfassbaren physiologischen Prozessen einhergehen (können). Wir begehen einen Kategorienfehler, wenn wir (nur) auf der Ebene der Neuro- und Kognitionswissenschaften umfassende Erklärungen für das Auftreten solcher phänomenaler Eigenschaften suchen. ${ }^{214}$

Im Zusammenhang mit der Behauptung, dass phänomenales Erleben vielleicht nur eine >Illusion< sei, ${ }^{215}$ wird nun versucht, die Unterscheidung von hard vs. easy problem zu verwischen. Bei ihrem Versuch, dies auszuweisen, gehen bspw. Mandik und Weisberg von einem Gedankenexperiment von Michael Lynch"16 aus. ${ }^{217}$ Im Gedankenexperiment setzt Lynch voraus, wir könnten uns vorstellen,

>auditorischen Gehirnbereich`? Vgl. dazu auch Roth, Gerhard: Das Gehirn und seine Wirklichkeit. Kognitive Neurobiologie und ihre philosophischen Konsequenzen, Frankfurt am Main: Suhrkamp 1994, S. 101.

211 Chalmers, David J.: The conscious mind. In search of a fundamental theory, New York: Oxford University Press 1996, S. 25 .

212 Bzw. dem, was Chalmers den »psychological mind « nennt. Ebd.

213 Das heisst dem, was Chalmers den »phenomenal mind « nennt. Das Problem besteht mit anderen Worten darin, dass wir keine Ahnung haben, wie bewusstes, phänomenales Erleben (»phenomenal mind«) überhaupt mit neurophysiologischen Eigenschaften (»psychological mind«) identisch sein könnte. Ebd.

214 Vgl. dazu Walter, Sven: »Allgemeine Einleitung: Phänomenales Bewusstsein: Unlösbares Mysterium oder seriöses wissenschaftliches Problem?«, in: Qualia. Ausgewählte Beiträge, Heckmann, Heinz-Dieter / Walter, Sven [Hrsg.], Paderborn: Mentis 2001, S. 24-25.

215 Vgl. dazu Mandik, Pete / Weisberg, Josh: »Type-q materialism«, 2008, Online: http://www. petemandik.com/philosophy/papers/typeq.pdf [zuletzt geprüft am 13.o9.2018], S. 16 .

216 Vgl. Lynch, Michael P.: »Zombies and the Case of the Phenomenal Pickpocket«, Synthese, [2006] Vol. 149, No. 1.

217 Vgl. dazu Dennett, Daniel C.: »Quining Qualia«, in: Philosophy of mind. Classical and contemporary readings, Chalmers, David J. [Hrsg.], New York: Oxford University Press [zuerst 1988]/2002, S. 231. 
dass es so etwas wie einen "phänomenalen Taschendieb « 218 gäbe, der - ohne dass wir dies »merken ${ }^{219}$ - Schritt für Schritt unser phänomenales Erleben stiehlt: »[...] so I will not detect a difference between being fully conscious and being without any conscious experience whatsoever ${ }^{220}$ Am Ende dieses Prozesses seien wir Zombies und hätten aber dennoch die Überzeugung, dass unsere Erlebnisse davon unberührt seien. ${ }^{221}$ Können wir uns das wirklich vorstellen? Wenn wir »without any conscious experience whatsoever « ${ }^{222}$ sind, was an einem solchen Organismus kann dann noch etwas nicht >merken<? Das Gedankenexperiment beruft sich dennoch auf die angebliche Möglichkeit, dass es ein >Nicht-Merken< einer Veränderung im Erleben geben könnte. Dajedoch >Merken< auf Auf-merk-samkeit basiert, kann es ohne Bewusstsein ${ }^{223}$ weder >Merken $<$ noch $>$ Nicht-Merken $<$ geben, sondern nur funktionale Abläufe.

218 Mandik, Pete / Weisberg, Josh: »Type-q materialism«, 2008, Online: http://www. petemandik.com/philosophy/papers/typeq.pdf [zuletzt geprüft am 13.09.2018], S. 20.

219 Lynch, Michael P.: »Zombies and the Case of the Phenomenal Pickpocket«, Synthese, [2006] Vol. 149, No. 1, S. 44.

220 Ebd. [Hervorhebung E. E.].

221 Vgl. ebd.

222 Ebd. Daniel Dennett argumentiert in einer ähnlichen Weise, indem er Beurteilung von Phänomenalem mit der sinnlichen Wahrnehmung von Phänomenalem verwechselt bzw. aus einer berechtigten Infragestellung der infalliblen Beurteilung von Qualia, irrtümlicherweise die `Existenz` von Phänomenalem zu >Widerlegen` glaubt. Eine phänomenale Wahrnehmung wird jedoch nicht durch deren fehlerhafte Beurteilung sinexistent«. Vgl. dazu Dennett, Daniel C.: »Quining Qualia«, in: Philosophy of mind. Classical and contemporary readings, Chalmers, David J. [Hrsg.], New York: Oxford University Press [zuerst 1988]/2002, S. 231. Auf die Problematik des Täuschungs-Argumentes gehe ich in Abschnitt 9.2.1 näher ein.

223 Man mag hier einwenden, dass Bewusstsein nicht gleichzusetzen ist mit phänomenalem Bewusstsein. Denn bspw. wird die Konzeption des Zugriffsbewusstseins von der des phänomenalen Bewusstseins unterschieden: die Bewusstheit eines Zustandes muss demnach nicht unbedingt mit dessen phänomenalem Charakter zusammenhängen. Nach dieser Konzeption gibt es auch phänomenale Zustände, die unbewusst sind. Vgl.: Van Gulick, Robert: »Consciousness «, in: The Stanford Encyclopedia of Philosophy, 2014, Online: http://plato.stanford.edu/archives/spr2014/entries/consciousness/ [zuletzt geprüft am 17.09.2018], Abschnitt 2.2. Aber im Gedankenexperiment geht es, mindestens in dessen Ausgangssituation, um ein bewusstes phänomenales Erleben bei welchem zu einem späteren Zeitpunkt angeblich nicht bemerkt würde, dass sich das Erleben verändert. Wenn im Gedankenexperiment also von einer Form des Zugriffsbewusstseins ausgegangen würde, so ändert sich für die Ausgangssituation des Gedankenexperimentes nichts. Denn nach der Konzeption des Zugriffsbewusstseins ist ein mentaler Zustand bewusst, wenn dessen Information funktional für den Organismus verfügbar ist. Wenn nun der Trick des >phänomenalen Taschendiebes` darin bestünde, dass er verhindert, dass die Information eines Erlebnisses für den Organismus nicht mehr verfügbar ist, dann würden wir dies gerade deshalb merken, weil wir etwas bestimmtes nicht mehr erleben. Das bedeutet, dass auch die Konzeption des `Zugriffsbewusstseins \& für die Behauptung, 
Um diese Strategie dieser Umdeutung des hard in ein easy problem jedoch darzustellen, nehmen wir aber einfach einmal an, dass wir uns tatsächlich vorstellen können, dass uns ein »Qualia Taschendieb « ${ }^{224}$ unser ganzes Erleben >raubt<, ohne dass wir dies »merken «.225 Falls das vorstellbar ist, ist es nach Lynch eben auch möglich, dass dies bereits passiert sein könnte und wir hier und jetzt überhaupt `keine Erlebnisse haben. ${ }^{226}$ Auf der Basis dieser im Gedankenexperiment implizierten Behauptung folgern Mandik und Weisberg: »Thus, however strongly it may seem to the subject that he or she has conscious experiences; he or she may very well be mistaken «. ${ }^{227}$ Im Hinblick auf die für eine phänomenal adäquate Theorie des Geistes so wichtige Unterscheidung zwischen dem hard und easy problem passiert also folgendes: auf der Basis von phänomenal inadäquaten Qualia-Konzeptionen ${ }^{228}$ und der

dass eine Veränderung im Erleben phänomenaler Zustände >nicht bemerktく werden könnte, nicht anwendbar ist.

Und wenn >Merken als reine Registrierfunktion oder funktionale Verfügbarkeit umgedeutet wird, die nichts mit dem Erleben der phänomenalen Zustände zu tun hat, dann würde im Gedankenexperiment von Lynch das, was mit ihm bewiesen werden soll, bereits vorausgesetzt: nämlich, dass es möglich sei, dass wir nicht >merken< könnten, wenn sich unser Erleben ändert. Es würde vorausgesetzt, dass wir noch gar nie etwas erlebt haben, weil das was wir >Erleben` nennen, nur eine Registrierfunktion von Zombies sei.

224 (Wörtlich »via phenomenal pick pocketing«) Mandik, Pete / Weisberg, Josh: »Type-q materialism «, 2008, Online: http://www.petemandik.com/philosophy/papers/typeq.pdf [zuletzt geprüft am 13.09.2018], S. 15 .

225 Lynch, Michael P.: »Zombies and the Case of the Phenomenal Pickpocket«, Synthese, [2006] Vol. 149, No. 1, S. 44.

226 Vgl. a.a.O., S. 46.

227 Mandik, Pete / Weisberg, Josh: »Type-q materialism«, 2008, Online: http://www. petemandik.com/philosophy/papers/typeq.pdf [zuletzt geprüft am 13.09.2018], S. 16. Die Überzeugung, dass wir bewusste Organismen sind, basiere deshalb nicht nur auf dem Erleben unserer eigenen Bewusstheit, sondern zusätzlich auf der Ablehnung der skeptischen Idee, dass es möglich sein könnte, dass wir (unbemerkt) nichts Erleben. Vgl. a.a.O., S. 16. Chalmers »taking consciousness seriously« (Vgl. Chalmers, David J.: The conscious mind. In search of a fundamental theory, New York: Oxford University Press 1996, S. xii.) sei aus diesem Grund - den Mandik und Weisberg mit der Duhem-Quine These in Verbindung bringen - angreifbar. Wenn man mit Chalmers das Explanandum des bewussten Erlebens ernst nehme und es als hard problem bezeichne, habe man gleichzeitig die Pflicht, jene skeptizistische Idee ernst zu nehmen, dass wir alle vielleicht bewusstlos sein könnten (und dies aber nicht merken). Vgl. dazu Mandik, Pete / Weisberg, Josh: »Type-q materialism«, 2008, Online: http://www.petemandik.com/philosophy/ papers/typeq.pdf [zuletzt geprüft am 13.09.2018], S. 16.

228 Vgl. dazu Abschnitte 8.1 und 9.2 
etwas eigentümlichen, ${ }^{229}$ gleichsam pyrrhonisch-skeptizistischen ${ }^{230}$ Behauptung, dass phänomenales Erleben, ja Erleben und Bewusstsein überhaupt, vielleicht nur eine >Illusion< sei, ${ }^{231}$ verwandelt sich das hard problem scheinbar in ein easy problem: »[...] once we properly acknowledge certain Quinean constraints, the situation for the materialist seems much brighter. We are left only with the ordinary >easy< problems of science «. ${ }^{232}$ So können wir feststellen, dass noch vor der Bewältigung des »halben Weges «, 233 hin zu einer brauchbaren Theorie des Mentalen - und m.E. auch mit Hilfe von Intuitionen über >zukünftige< Lösungen ${ }^{234}$ des hard problem - Zuflucht beim easy problem und der Erklärung von funktionalen Zusammenhängen gesucht wird.

Wenn wir den Hoffnungen auf irgendwelche zukünftigen Lösungen des hard problem weniger Glauben entgegenbringen können, so wirft ein kritischer Blick auf gegenwärtige Theorien des Geistes eher die Frage auf, ob nicht nur das hard, sondern auch das easy problem unterschätzt wird. Denn selbst

229 Eigentümlich, weil der Begriff der >Illusion< nur Sinn macht, wenn >Illusion das von Subjekten erlebt werden kann. Denn wer oder was, ausser ein Subjekt, kann eine >Illusion haben oder sich >in Illusionen wiegen<? Ein Taschenrechner, der ein falsches Resultat liefert, ist entweder falsch programmiert oder defekt; aber dass der Taschenrechner >einer Illusionen unterliegt<, wird wohl niemand ernsthaft behaupten. Wenn nun also behauptet wird, dass das Erleben selbst eine >Illusion< sein könnte, so wirkt dies insofern unsinnig, weil unter der Voraussetzung, dass es kein Erleben >gibt<, es auch nicht so etwas, wie eine >Illusion` geben kann. Das bedeutet, dass sich die Behauptung `Erleben ist eine Illusion< selber aufhebt, weil sie von einer Voraussetzung ausgeht, die in der Behauptung zugleich geleugnet wird.

230 Ich nenne diese Form des Skeptizismus, die davon ausgeht, dass Erleben, ja Bewusstsein überhaupt, vielleicht nur eine >Illusion`sein könnte, deshalb >pyrrhonisch<, weil die Frage >erlebe ich jetzt wirklich oder erlebe ich nur die Illusion, dass ich erlebe oder erlebe ich nicht einmal diese Illusion, obwohl es so scheint? ähnlich radikal Skeptizistisch ist, wie die Frage >denke ich jetzt wirklich oder täuschen mich meine Gedanken nur darüber, dass ich jetzt denke oder denke ich nicht einmal diese täuschenden Gedanken, obwohl es so scheint?«. Nach Nagel ist letztere Art des radikalen Skeptizismus aus folgendem Grund unhaltbar bzw. undenkbar: »Gäbe es mögliche Wesen, deren Natur und Weltbeziehung so beschaffen wären, dass, was auch immer in ihnen vorginge, nichts davon ein Gedanke sein könnte, dann könnte ich mich gar nicht fragen, ob ich ein solches Wesen bin, denn wäre ich es, so würde ich nicht denken, und auch die blosse Erwägung der Möglichkeit, dass ich nicht denke, ist bereits ein Gedanke«. Nagel, Thomas: Der Blick von nirgendwo, Frankfurt am Main: Suhrkamp [zuerst 1986, engl.]/1992, S. 128, Fussnote Nr. 4.

231 Vgl. dazu Mandik, Pete / Weisberg, Josh: »Type-q materialism«, 2008, Online: http://www. petemandik.com/philosophy/papers/typeq.pdf [zuletzt geprüft am 13.o9.2018], S. 16.

232 A.a.O., S. 18 [Hervorhebung E. E.].

233 Elitzur, Avshalom C.:»Consciousness makes a difference:A reluctant dualist's confession«, 2009, Online: http://philpapers.org/archive/ELICMA.pdf [zuletzt geprüft am 13.09.2018], S. 4.

234 Vgl. dazu Abschnitt 4.2.5. 
mentale Phänomene, die allgemein dem easy problem zugerechnet werden, sind nicht zwingend so unproblematische Explananda, wie es a prima vista erscheinen mag. Im Hinblick auf die Behauptung, dass sich intentionale Eigenschaften des Mentalen leichter naturalisieren lassen als phänomenale Eigenschaften, ${ }^{235}$ lässt sich einwenden, dass Phänomenalität und Intentionalität unentwirrbar verbunden sind. ${ }^{236}$ Mit der Kritik an der herkömmlichen Unterscheidung zwischen Phänomenalem und Intentionalem, drängt sich sogar die Vermutung auf, dass selbst das easy problem nicht so einfach ist, wie es für Naturalisierungsprogramme wünschenswert wäre: »This inextricable linkage between phenomenology and intentionality in emotion will, I suspect, make the so-called >easy problem < hard, and not the so-called `hard problem< easy «. ${ }^{237}$

\subsubsection{Patt-Situation und Beweislastabwehr}

Ein weiteres Problem im Umgang mit dem hard problem ist die aus einer Patt-Situation resultierende Tendenz der Beweislastabwehr bzw. der gegenseitigen Beweislastzuschiebung. ${ }^{238}$ Diesbezüglich stellt Walter fest: »des Einen Modus Ponens ist des Anderen Modus Tollens [sic!]«.239 Der modus tollens der Physikalisten sieht dabei folgendermassen aus:

Prämisse 1: Wenn phänomenale von physikalischen oder funktionalen Eigenschaften ( $p$-Eigenschaften) nicht zwingend abhängig sind, dann können phänomenale Eigenschaften nicht mit $p f$-Eigenschaften identisch sein.

235 Die Erklärung von intentionalen mentalen Eigenschaften, die solchen Annahmen gemäss, von phänomenalen Eigenschaften unterschieden werden, werden in diesem $\mathrm{Zu}$ sammenhang dementsprechend dem easy problem zugeordnet. Vgl. dazu auch Graham, George / Horgan, Terence / Tienson, John: »Phenomenology, Intentionality, and the Unity of the Mind «, in: The Oxford Handbook of Philosophy of Mind, McLaughlin, Brian P. I Beckermann, Ansgar / Walter, Sven [Hrsg.], Oxford: Oxford University Press 20o9, S. 534.

236 Nach Goldie sind Wahrnehmungszustände wie >rot< oder ein Gefühl wie >gefährlich< im Kern zugleich phänomenal und intentional (»essentially phenomenological and essentially intentional«). Goldie, Peter: »Emotions, feelings and intentionality«, Phenomenology and the Cognitive Sciences, [2002] Vol. 1, No. 3, S. 250. Mehr über die intentionalen Aspekte des Phänomenalen vgl. Abschnitt 8.2.5

237 Ebd. [Hervorhebung E. E.].

238 Vgl. dazu auch Schildknecht, Christiane: »Der Dualismus und die Rettung der Phänomene «, in: Homo Sapiens und Homo Faber. Epistemische und technische Rationalität in Antike und Gegenwart. Festschrift für Jürgen Mittelstrass, Wolters, Gereon / Mittelstrass, Jürgen [Hrsg.], Berlin: W. de Gruyter 2005, S. 225.

239 Walter, Sven: »Allgemeine Einleitung: Phänomenales Bewusstsein: Unlösbares Mysterium oder seriöses wissenschaftliches Problem?«, in: Qualia. Ausgewählte Beiträge, Heckmann, Heinz-Dieter / Walter, Sven [Hrsg.], Paderborn: Mentis 20o1, S. 53. 
Prämisse 2: Phänomenale und $p f$-Eigenschaften sind identisch.

Konklusion: Phänomenale Eigenschaften können nicht unabhängig von $p f$ Eigenschaften sein. ${ }^{240}$

\section{Der modus ponens der »Qualia-Freunde« ist demgegenüber so:}

Prämisse 1: Wenn phänomenale von $p f$-Eigenschaften nicht zwingend abhängig sind, dann können phänomenale Eigenschaften nicht mit $p f$-Eigenschaften identisch sein.

Prämisse 2: Phänomenale und $p f$-Eigenschaften sind nicht zwingend voneinander abhängig.

Konklusion: Phänomenale Eigenschaften können nicht mit $p f$-Eigenschaften identisch sein. ${ }^{241}$

Weiter stellt Walter fest, dass das Fazit von nun mehr als fünfzig Jahren analytischer Philosophie des Geistes sei, dass weder Reduktionismus noch AntiReduktionismus zwingend Gültigkeit für sich beanspruchen können. ${ }^{242}$

Im Rahmen dieser Dissertation werfe ich die Frage auf, ob sich das »Patt« bei einem phänomenal adäquaten Qualia-Begriff zu ungunsten von Reduktionsoptimisten verschieben könnte. Auch wenn es einfacher sein mag, innerhalb der oben geschilderten Default-Positionen zu argumentieren, sind Physikalisten in keiner bequemen Lage. Denn sie berufen sich auf negative Existenzbehauptungen. ${ }^{243}$ Genauer: sie behaupten, dass es keine Argumente gebe, die zeigen, dass der Physikalismus als Theorie des Geistes sich unmöglich halten

240 Vgl. a.a.O., S. 54. Sven Walter verwendet in Prämisse 1 den Begriff »unabhängig«. Dieses Argument ist jedoch genauer gefasst, wenn Qualia und $p f$-Eigenschaften nicht zwingend voneinander abhängig sind. Dass es um eine mögliche Unabhängigkeit geht, und somit um ein modales Argument, zeigt auch Walters Ergänzung zum Begriff >unabhängig`in seiner Fussnote 41: »Wenn es möglich (und nicht nur vorstellbar) ist, dass phänomenale Eigenschaften instantiiert sind, ohne dass die entsprechenden $p f$-Eigenschaften instantiiert sind $[\ldots] \ll$. Ebd.

241 Vgl. ebd.

242 Walter spricht von fünfzig Jahren, inzwischen sind dies schon mehr als 6o Jahre. Obwohl nach Walter nicht feststeht, ob phänomenales Bewusstsein ein unlösbares Mysterium ist, gesteht er dennoch, trotz dem diagnostizierten »Patt« ein, dass phänomenales Bewusstsein ein $»[\ldots]$ seriöses philosophisches, wie auch empirisches Problem [...]« darstellt. A.a.O., S. 56 .

243 Vgl. dazu auch a.a.O., S. 55. Dazu kommen noch die oben erwähnten, problematischen negativen Existenzbehauptungen von eliminativistischen Physikalisten über die angebliche $>$ Nicht-Existenz $\prec$ von Erleben. 
lasse. ${ }^{244}$ Solche negativen Existenzbehauptungen lassen sich nach Walter jedoch notorisch schwer beweisen. ${ }^{245}$ Genau betrachtet sind diese negativen Existenzbehauptungen sogar ziemlich nutzlos, denn Reduktionsskeptiker/ innen können entgegnen, dass es ebenso schwer ist, das Gegenteil zu beweisen. Anders gesagt: es gibt keine Argumente, die zeigen, dass es unmöglich ist, dass der reduktive Physikalismus als Theorie des Geistes falsch ist.

Mit einem kritischen Blick auf die versteckten Prämissen in physikalistischen Argumenten zeigt sich dementsprechend, dass Physikalisten gegenüber reduktionskritischen Theorien des Geistes im besten Fall ein Patt aufzeigen können. Beispielsweise konstatiert Walter, dass ein Physikalist argumentieren könnte, dass aus der Tatsache, dass wir »einen Gegenstandsbereich $\ll^{246}$ auf zwei verschiedene, nicht aufeinander reduzierbare Arten und Weisen konzeptualisieren, keine ontologischen Schlussfolgerungen gezogen werden dürften. Auf eine analoge Weise argumentiert Loar, der im Zusammenhang mit phänomenalen bzw. physikalischen Begriffen zwar zwei »epistemische Relationen $\ll^{247}$ eingesteht, jedoch davon ausgeht, dass sich diese nur auf physikalisch-funktionale Tatsachen beziehen. Genau betrachtet, stehen solche Argumente ontologischen Schlussfolgerungen nur vermeintlich kritisch gegenüber. Der Ausdruck >einen Gegenstandsbereich`, den z.B. Walter verwendet, zeigt nämlich, dass mit diesem Argument selbst voreilig eine ontologische Schlussfolgerung gemacht wird: nämlich der Schluss, dass die Prämisse

244 Auf negativen Existenzbehauptungen (im Hinblick auf Argumente) basiert z.B. Loars >Begründung 〈, dass Erleben mit Physikalischem identisch sei: »Wie ich die Sache sehe, gibt es kein überzeugendes philosophisch artikuliertes Argument, das das Gegenteil zeigen würde«. Loar, Brian: »Phänomenale Zustände«, in: Begriff, Erklärung, Bewusstsein. Neue Beiträge zum Qualia-Problem, Pauen, Michael / Schütte, Michael / Staudacher, Alexander [Hrsg.], Paderborn: Mentis [zuerst 1997, engl.]/2007, S. 29. Auch Churchland beruft sich darauf, dass bis jetzt noch nicht die Falschheit von physikalistischen Konzeptionen des Bewusstseins bewiesen worden sei. Vgl. Churchland, Paul M.: »The Rediscovery of Light«, The Journal of Philosophy, [1996] Vol. 93, No. 5, S. 224.

245 Vgl. Walter, Sven: »Allgemeine Einleitung: Phänomenales Bewusstsein: Unlösbares Mysterium oder seriöses wissenschaftliches Problem?«, in: Qualia. Ausgewählte Beiträge, Heckmann, Heinz-Dieter / Walter, Sven [Hrsg.], Paderborn: Mentis 20o1, S. 55.

246 A.a.O., S. 31 [Hervorhebung E. E.].

247 »Only physical-functional facts are involved, but there are two epistemic relations to them«. Loar, Brian: »Phenomenal states«, in: Philosophy of mind. Critical concepts in philosophy. Volume 4: Consciousness, Crawford, Sean [Hrsg.], London: Routledge \& Kegan Paul [zuerst 199o]/2011, S. 51 [Hervorhebung im Original]. Vgl. auch die Stelle, bei der Loar von phänomenalen und functional-physikalischen Begriffen spricht, dabei aber die Vermutung äussert, dass beide Begriffsarten auf physikalische Eigenschaften referieren: $\gg$ The property to which a phenomenal concept somehow directly refers may just be a property captured by a term of a physical-functional theory«. A.a.O., S. 5०. 
eines strikt monistischen Materialismus die Pattsituation zu Gunsten der Reduktionsoptimisten entscheidet. ${ }^{248}$ So behauptet auch Loar, introspektive Begriffe könne man zwar akzeptieren, dennoch könne man Erleben mit neuronalen Eigenschaften identifizieren. ${ }^{249}$ Auch bei Block findet man Passagen, die sich auf diese Argumentationsform der Beweislastverschiebung stützen. Er gesteht zwar ein, dass es subjektives Erleben bzw. Begriffe gibt, die sich nicht aus einer Dritte-Person-Perspektive aneignen lassen. Ein Physikalist könne folglich ein nicht-physikalistisches Vokabular zulassen. ${ }^{250}$ Dies zeige jedoch nicht, dass es neben den physikalischen Tatsachen noch irgendwelche anderen Tatsachen gebe. ${ }^{251}$

Die Beweislastzuschiebung besteht also darin, dass sich reduktionsoptimistische Positionen darauf zurückziehen, dass das von ihnen anerkannte ${ }^{252}$

248 Diesbezüglich stellt Marienberg fest, dass es »[...] schon vor dem Versuch ihrer materialistischen oder funktionalistischen Interpretation oder auch dem kategorialen Verzicht auf solche Deutungsversuche [...] « davon abhängt, ob phänomenale Bewusstseinszustände wissenschaftlich angemessen erfasst werden können. Marienberg, Sabine: »Die Grenzen der Vergegenständlichung qualitativen Erlebens«, in: Funktionen des Erlebens. Neue Perspektiven des qualitativen Bewusstseins. Band 5: Humanprojekt, Jung, Matthias / Heilinger, Jan-Christoph [Hrsg.], Berlin: De Gruyter 2009, S. 42. Für sie verhindern Vorentscheidungen, die phänomenale Bewusstseinszustände als »[...] Ärgernis, Wunder oder schlicht inexistent betrachten $[\ldots]$ « dass sie überhaupt zu einem Gegenstand systematischer Untersuchungen werden können. Vgl. a.a.O., S. 43. Allerdings kritisiert Marienberg auch Vorentscheidungen, die Phänomenales im Vorhinein als ineffabel und intersubjektiv unerreichbar deuten würden. Vgl. a.a.O., S. 42-43.

249 Loar räumt also nur ein, dass phänomenale Begriffe begrifflich irreduzibel seien. D.h., dass sie weder physisch-funktionale Begriffe implizieren noch von diesen impliziert werden. Vgl. Loar, Brian: »Phänomenale Zustände«, in: Begriff, Erklärung, Bewusstsein. Neue Beiträge zum Qualia-Problem, Pauen, Michael / Schütte, Michael / Staudacher, Alexander [Hrsg.], Paderborn: Mentis [zuerst 1997, engl.]/2007, S. 28.

250 Vgl. Block, Ned J.: »Das schwierigere Problem des Bewusstseins«, in: Anatomie der Subjektivität. Bewusstsein, Selbstbewusstsein und Selbstgefühl, Grundmann, Thomas / Hofmann, Frank / Misselhorn, Catrin et al. [Hrsg.], Frankfurt am Main: Suhrkamp [zuerst 2002, engl.]/2005, S. 46.

251 Vgl. ebd. Auch bei Tye findet sich diese Argumentationsform. Nach ihm sind bei der explanatorischen Lücke lediglich »verschiedene Begriffe über physikalische Zustände« (»different ways of conceiving certain physical states«) im Spiel. Tye, Michael: Ten problems of consciousness. A representational theory of the phenomenal mind, Cambridge, Massachusetts: MIT Press 1995, S. 180. Durch diese verschiedenen Begriffe über physikalische Zustände entstehe dann die Illusion, dass von verschiedenen Dingen die Rede sei. Vgl. dazu auch Staudacher, Alexander: Phänomenales Bewusstsein als Problem für den Materialismus, Berlin: De Gruyter 2002, S. $35^{8}$.

252 Radikale reduktionistische Positionen, wie der Eliminativismus, können sich in Kombination mit der Behauptung, dass es nur so >scheine`, dass Erleben existiert, darauf berufen, dass der Eindruck, es gäbe dieses Explanandum, nicht zeige, dass es phänomenale 
Explanandum `Erleben $<$ zwar existiert, das dies aber, trotz aller Misserfolge, es zu reduzieren, nicht zeige, dass Erleben irreduzibel sei. Das Problem, dass dies mindestens ${ }^{253}$ ebenso wenig das Gegenteil (dass Erleben reduzierbar ist) zeigt, wird dabei offenbar unterschlagen.

Interessant ist im Hinblick auf die Tendenz der Beweislastabschiebung auch eine Passage bei Metzinger, der freilich nicht ohne Grund die »trügerische Selbstgewissheit der Introspektion « ${ }^{254}$ anzweifelt: denn bestimmte Experimente demonstrieren, dass sich das auf den Körper bezogene Ichgefühl unter experimentellen Bedingungen selektiv beeinflussen lässt. ${ }^{255}$ Aus solchen und anderen Fallibilitätsbeispielen folgert Metzinger, dass jene »trügerische Selbstgewissheit der Introspektion ${ }^{256}$ unter dem Ansturm von neuem empirischem Wissen über die »neuronale Maschinerie «, ${ }^{257}$ die im Rücken des Erlebnissubjektes agiert, »endgültig zerbrochen ${ }^{258}$ ist. Uns bleibe deshalb nur ein $»$ vorurteilsfreies tieferes Eindringen ${ }^{259}$ in die theoretischen Probleme der Subjektivität. In diesem Zusammenhang wirft Metzinger die Frage auf, ob das Subjekt, in einem »philosophisch interessanten Sinn von $>$ Wissen « «260 überhaupt um seine eigene Bewusstheit weiss und ob der Gehalt phänomenaler Zustände eine »Form von Wissen $\ll^{261}$ darstellt.

Tatsachen gibt. Vgl. z.B. Dennett, Daniel C.: Consciousness explained, London: Lane 1991, S. 98.

253 Wenn wir bedenken, dass von moderaten Positionen das Explanandum `Erleben<, für welches bisher kein zufrieden stellendes Explanans gefunden wurde, akzeptiert wird, so stellt sich die Frage, ob diese Misserfolge die Beweislast den reduktionsoptimistischen Zugängen aufbürdet.

254 Metzinger, Thomas: Subjekt und Selbstmodell. Die Perspektivität phänomenalen Bewusstseins vor dem Hintergrund einer naturalistischen Theorie mentaler Repräsentation, Paderborn: Mentis 1999, S. 38.

255 Experimente von Olaf Blanke und Bigna Lenggenhager und Tej Tadi demonstrieren, dass das ganzheitliche Ichgefühl sich unter experimentellen Bedingungen selektiv beeinflussen lässt. Mit Hilfe von Techniken der virtuellen Realität hatten Versuchspersonen in diesen Experimenten das Gefühl, ausserhalb ihres Körpers zu sein bzw. sie identifizierten sich während der Manipulation mit einem computergenerierten Bild des eigenen Körpers. Vgl. Metzinger, Thomas: Der Ego Tunnel. Eine neue Philosophie des Selbst: Von der Hirnforschung zur Bewusstseinsethik, Berlin: Berliner Taschenbuch Verlag [zuerst 2009, engl.]/2011, S. 21.

256 Metzinger, Thomas: Subjekt und Selbstmodell. Die Perspektivität phänomenalen Bewusstseins vor dem Hintergrund einer naturalistischen Theorie mentaler Repräsentation, Paderborn: Mentis 1999, S. 38 .

257 Ebd.

258 Ebd.

259 Ebd.

260 A.a.O., S. 37.

261 A.a.O., S. 38. 
Doch auch bezüglich dieses Argumentationsstranges mit Fallibilitätsbeispielen besteht genau betrachtet eine Patt-Situation. Nur wenn dies nicht gesehen wird, können diese Beispiele für die übliche Beweislastzuschiebung an Reduktionskritiker verwendet werden. Denn die von Metzinger geforderte »Vorurteilsfreiheit « ${ }^{262}$ besteht erstens darin anzuerkennen, dass die Fallibilität des Erlebens im Hinblick auf Urteile über seine Gehalte nicht zeigt, dass der Bereich des Phänomenalen befriedigend auf eine »neuronale Maschinerie « 263 reduziert werden kann. Zweitens ist der Nachweis der Manipulierbarkeit von Erleben nicht mit einem Beweis gleichzusetzen, dass es identisch mit neuronalen Vorgängen ist. ${ }^{264}$ Drittens besteht Vorurteilsfreiheit gerade auch darin, kritisch auf eine vielleicht ebenso »trügerische ${ }^{265}$ Gewissheit zu schauen, dass bewusstes Erleben irgendwann in der Zukunft vollständig auf neurophysiologische Vorgänge reduziert und dann sicher lückenlos und befriedigend erklärt werden könne. Viertens zeigt die Möglichkeit der Fallibilität im Erleben unsers Ich-Gefühls nicht auf, dass wir gar nichts erleben und Phänomenales deshalb kein Explanandum sein soll.

Bei Crane wiederum zeigt sich die Beweislastverschiebung in der Art und Weise, wie er über den Qualia-Begriff von Reduktionsskeptiker/innen räsoniert. Er behauptet, dass ein Begriff des Phänomenalen, bei dem man davon ausgehe, dass Phänomenales nicht mit einem >Hirnzustand identifizierbar sei, eine »mere assertion $\aleph^{266}$ sei - dass jedoch die Hoffung auf eine >künftige<, vollständige Identifizierbarkeit von Phänomenalem mit Hirnzuständen genauso auf >blossen< bestimmten Grundannahmen beruht, verschweigt er. Crane gesteht zwar ein, dass physikalisches Wissen kein Wissen über Qualia begründen könne. Nichtsdestotrotz gibt Crane keine Begründung dafür an, warum diese Wissenslücke nicht für eine irreduzible Eigenschaft der phänomenalen $\mathrm{Zu}$ stände sprechen könnte. ${ }^{267}$

Fazit: Argumentationslinien, die auf einer Beweislastzuschiebung fussen, sind nur solange befriedigend, wie diese Beweislastzuschiebung nicht in Frage gestellt bzw. nicht bemerkt wird. So wird zuweilen von strikt monistischen

\footnotetext{
262 Ebd.

263 Ebd.

264 Oder dass deshalb reduktive Theorien des Geistes von der Beweislast ihrerseits befreit sein sollen.

265 Ebd.

266 Crane, Tim: »The origins of qualia«, 200o, Online: http://sas-space.sas.ac.uk/220/1/ The\%2oorigins\%2oof\%2oqualia.pdf [zuletzt geprüft am 24.09.2018], S. 23.

267 A.a.O., S. 1-33. Ein weiteres Beispiel der Beweislastzuschiebung findet sich auch bei Papineau, vgl. Papineau, David:»Mind the Gap«, Philosophical Perspectives, [1998] Vol. 12: Language, Mind, and Ontology, S. 374 und 379.
} 
Materialisten das zu Beweisende ebenso vorausgesetzt, wie beispielsweise ein immaterieller Idealismus oder ein Solipsismus seine spezifische Ontologie einfach voraussetzt. Demgemäss `gibt $<$ es beim immateriellen Idealismus per Definition letztlich nur »Geistiges «.268 Analog wird bei den erwähnten Argumentationsformen mit Beweislastzuschiebung behauptet, dass Erleben physikalisch sein muss, weil das, was existiert eben per Definition nur etwas sein kann, das etwas >rein Physikalisches< im herkömmlichen Sinne ist.

\subsubsection{Einschüchterung von Reduktionsskeptikern/innen}

Bei der Durchsicht der Forschungsliteratur fiel mir auf, dass - in Kombination mit der erwähnten Tendenz der Beweislastabschiebung - reduktionskritische Zugänge zum hard problem begrifflich so konnotiert werden, dass dies bewusst oder unbewusst als >Einschüchterung der Gegner wirken kann.

In reduktionsoptimistischen Argumentationssträngen fällt der Begriff >Intuition< auffällig oft. So bringt Metzinger die Konzeption von Phänomenalem bzw. Qualia in Verbindung mit einer »essentialistischen Intuition «269 an anderer Stelle verweist er darauf, dass Theorien des Geistes, die das Phänomenale ernst nehmen, einer »cartesianischen Intuition $\aleph^{270}$ auf den Leim gingen. ${ }^{271}$ Mandik stützt sich in seiner Argumentation ebenfalls auf eine angeblich »pretheoretic intuition $\ll, 272$ die dafür verantwortlich sei, dass wir noch nicht, wie die »Kinder der Zukunft«, ein funktionales, neurophysiologisches Verständnis des Phänomenalen so natürlich, wie die Theorie der Gravitation fänden. ${ }^{273}$ Auch Beckermann verweist auf Intuitionen: 1. Die Annahme, dass es Qualia gebe, sei einfach eine »starke Intuition ${ }^{274}$ aber keine Intuition sei sakrosankt. 2. Wenn wir an dieser »Intuition« festhalten wollen, ergäben sich

268 So beschreibt Guyer die monistisch-immaterielle Form des Idealismus (im Gegensatz zum epistemischen Idealismus) folgendermassen: $»[\ldots]$ something mental (the mind, spirit, reason, will) is the ultimate foundation of all reality, or even exhaustive of reality $[\ldots ..] \ll$. Guyer, Paul / Horstmann, Rolf-Peter: »Idealism«, in: The Stanford Encyclopedia of Philosophy, 2015, Online: http://plato.stanford.edu/archives/fall2015/entries/idealism/ [zuletzt geprüft am 26.og.2016], Abschnitt 1.o.

269 Metzinger, Thomas: Subjekt und Selbstmodell. Die Perspektivität phänomenalen Bewusstseins vor dem Hintergrund einer naturalistischen Theorie mentaler Repräsentation, Paderborn: Mentis 1999, S. 36 .

270 A.a.O., S. 218.

271 Vgl dazu auch a.a.O., S. 19, 29 und 213.

272 Mandik, Pete / Weisberg, Josh: »Type-q materialism«, 2008, Online: http://www. petemandik.com/philosophy/papers/typeq.pdf [zuletzt geprüft am 13.09.2018], S. 2 .

273 Vgl. a.a.O., S. 2 o.

274 Beckermann, Ansgar: Analytische Einführung in die Philosophie des Geistes, Berlin: W. de Gruyter 2008, S. 454. 
dabei »erhebliche theoretische Probleme $\ll, 275$ deshalb sollen wir uns fragen, ob es nicht gute Gründe gebe, unsere »Intuition « über die »Existenz « ${ }^{276}$ von Qualia zu bezweifeln. Dass sich ebenfalls starke theoretische Probleme ergeben, wenn Qualia geleugnet werden (wie das modale, das epistemische und das explanatorische Argument zeigen), wird - entsprechend der Haltung des Abschiebens der Beweislast - weniger in Betracht gezogen.

Eine weitere Variante ist die von Loar erwähnte "phänomenologische Intuition « ${ }^{277}$ Loar behauptet diesbezüglich, dass jemand, der nicht seine Ansicht teilt, dass Erleben mit neuronalen Eigenschaften identifizierbar sei,

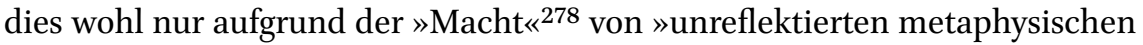
Intuition[en] $\ll^{279}$ tue.

Wenden wir solche Begrifflichkeiten jedoch konsequent an, d.h. auf beiden Seiten der Zugänge ${ }^{280}$ zum hard problem, so offenbart sich das Tendenziöse dieses Sprachgebrauchs. Wir könnten dann Loars Behauptung wie folgt reformulieren: >Wenn jemand die Ansicht teilt, dass Erleben und Physikalisches identisch sind, dann liegt dies wohl an der Macht einer reduktionsoptimistischen Intuition ${ }^{281}$ Wenn nun behauptet wird, dass nur die Reduktionsskeptiker/innen einer »Intuition « unterliegen, und dieser »Intuition« (wohl infolge einer schwer zu leugnenden Plausibilität) erst noch unterstellt wird, dass sie einer $»$ Macht ${ }^{2}{ }^{282}$ unterliegen soll, so ist dies ziemlich tendenziös. Paradoxerweise behauptet aber ausgerechnet Loar, dass dem

\section{Ebd.}

276 Ebd.

277 Loar, Brian: »Phänomenale Zustände«, in: Begriff, Erklärung, Bewusstsein. Neue Beiträge zum Qualia-Problem, Pauen, Michael / Schütte, Michael / Staudacher, Alexander [Hrsg.], Paderborn: Mentis [zuerst 1997, engl.]/2007, S. 28.

278 A.a.O., S. 29.

279 Ebd.

280 Damit meine ich sowohl reduktionskritische als auch den reduktionistische Zugänge zum hard problem.

281 Kein Zitat, sondern die Umkehrung von Loars Behauptung. Vgl. ebd. Selbstverständlich sind im Streit um das hard problem immer Intuitionen im Spiel. Dies betrifft jedoch Argumente aller Positionen, da sie letztlich alle auf bestimmten Prämissen fussen: »There is no denying that this involves an appeal to intuition at some point; but all arguments involve intuition $[. ..] \ll$. Chalmers, David J.: The conscious mind. In search of a fundamental theory, New York: Oxford University Press 1996, S. xii. Vgl. dazu auch Chalmers, David J:: »Bewusstsein und sein Platz in der Natur«, in: Grundkurs Philosophie des Geistes. Band 1: Phänomenales Bewusstsein, Metzinger, Thomas [Hrsg.], Paderborn: Mentis [zuerst 2002, engl.]/2007, S. 134 .

282 Loar, Brian: »Phänomenale Zustände«, in: Begriff, Erklärung, Bewusstsein. Neue Beiträge zum Qualia-Problem, Pauen, Michael / Schütte, Michael / Staudacher, Alexander [Hrsg.], Paderborn: Mentis [zuerst 1997, engl.]/2007, S. 29. 
Physikalismus gegenüber skeptische Argumente die »Rationalisierung einer Intuition ${ }^{283}$ seien, deren Kraft die Tatsache verberge, dass sie »tendenziös « ${ }^{284}$ seien.

Die Einschüchterung, ob bewusst oder unbewusst, besteht also in folgendem: Die Ansichten derjenigen, die skeptisch sind, ob sich Erleben tatsächlich so leicht mit neuronalen Eigenschaften identifizieren lässt, werden nicht nur als >Intuition<, sondern zudem als falsche bzw. »vortheoretische «, 285 »cartesianische $«^{286}$ oder $»$ unreflektierte ${ }^{287}$ Intuitionen herabgesetzt. Man deutet also mit einem ganz anderen Massstab die eigenen reduktionsoptimistischen Intuitionen als diejenigen Intuitionen der anderen (reduktionskritischen) Patt-Seite.

Im Zusammenhang mit der einschüchternden Verwendungsweise des Begriffs >Intuition< findet sich nicht selten auch eine damit einhergehende (implizite) Herabsetzung des philosophischen Antipoden, die darin besteht, zu behaupten, dass es einfach nur so »scheine $\ll,{ }^{288}$ als ob es Erleben gibt. Diese Deutung verwendet bspw. Dennett gegen Chalmers: »One central problem<, Chalmers tells us, >is that consciousness seems to be a further fact about conscious systems< [...]. He is right, so long as we put the emphasis on $>$ seems $\ll{ }^{289}$ So erstaunt es auch nicht, dass Dennett davon ausgeht, dass seine

283 A.a.O., S. 28 .

284 Ebd.

285 Mandik, Pete / Weisberg, Josh: »Type-q materialism«, 2008, Online: http://www. petemandik.com/philosophy/papers/typeq.pdf [zuletzt geprüft am 13.09.2018], S. 20. Auch Dennett sucht Zuflucht in dieser Begrifflichkeit im Zusammenhang mit seiner Behauptung, dass die Unterscheidung zwischen phänomenalem Erleben und physiologischen Zuständen einfach eine »vortheoretische Konstruktion« (»pretheoretical construal«) sei. Dennett, Daniel C.: »Quining Qualia«, in: Philosophy of mind. Classical and contemporary readings, Chalmers, David J. [Hrsg.], New York: Oxford University Press [zuerst 1988]/2002, S. 229.

286 Metzinger, Thomas: Subjekt und Selbstmodell. Die Perspektivität phänomenalen Bewusstseins vor dem Hintergrund einer naturalistischen Theorie mentaler Repräsentation, Paderborn: Mentis 1999, S. 218.

287 Loar, Brian: »Phänomenale Zustände «, in: Begriff, Erklärung, Bewusstsein. Neue Beiträge zum Qualia-Problem, Pauen, Michael / Schütte, Michael / Staudacher, Alexander [Hrsg.], Paderborn: Mentis [zuerst 1997, engl.]/2007, S. 29.

288 So >scheint< es nach Dennetts Heterophänomenologie nur so, als ob Erlebnisse existieren. Vgl. Dennett, Daniel C.: Consciousness explained, London: Lane 1991, S. 98. Vgl. dazu auch Metzinger, Thomas [Hrsg.]: Grundkurs Philosophie des Geistes. Band 1: Phänomenales Bewusstsein, Paderborn: Mentis 2007, S. 102-103.

289 Dennett, Daniel C.: »The Mystery of David Chalmers«, Journal of Consciousness Studies, [2012] Vol. 19, No. 1-2, S. 88. 
Theorie >des Geistes« die »Kurzsichtigkeit «290 von Philosophen, die nicht so reduktionsoptimistisch wie er sind, korrigieren könne. Diesbezüglich bedient er sich sogar des noch polemischeren und einschüchternderen Begriffs einer »kognitiven Behinderung «, ${ }^{291}$ die korrigiert werden könne. Zugleich beruft er sich darauf dass seine Deutung des hard problem mit »science «292 identisch sei:

[...] a counter-intuitive theory like mine still has an advantage over some of the fantasies in which philosophers have recently indulged. [...] we are all snatural born dualists«, but just as eyeglasses can correct for myopia, natural-born or not, so science can correct for this innate cognitive disability. ${ }^{293}$

Ebenfalls auf einer Gegenüberstellung von angeblich durchgängig »naiven« «94 versus reduktionistischen Konzeptionen von Bewusstsein basieren die einschüchternden Begriffe »folk psychology«,295 »folk theory « 296 oder »folk convictions « ${ }^{297}$ Der Dünkel von Positionen, die jene plakativen Gegenüberstellungen ${ }^{298}$ verwenden, stützt sich offenbar auf die in Abschnitt 6.4

290 Dennett, Daniel C.: »Why and How Does Consciousness Seem the Way it Seems?«, 2015, Online: http://open-mind.net/papers/why-and-how-does-consciousness-seem-the-wayit-seems [zuletzt geprüft am 24.09.2018], S. 10.

291 A.a.O., S. 10.

292 A.a.O., S. 10.

293 A.a.O., S. 10 [Hervorhebung E. E.].

294 »naïve conception of consciousness « Mandik, Pete / Weisberg, Josh: »Type-q materialism«, 2008, Online: http://www.petemandik.com/philosophy/papers/typeq.pdf [zuletzt geprüft am 13.09.2018], S. 20.

295 Ramsey, William: »Eliminative Materialism«, in: The Stanford Encyclopedia of Philosophy, 2013, Online: http://plato.stanford.edu/archives/sum2013/entries/materialismeliminative/ [zuletzt geprüft am 25.09.2018], Abschnitt 3.o. Vgl. dazu auch Churchland, Paul M.: »Eliminative Materialism and the Propositional Attitudes«,Journal of Philosophy, [1981] Vol. 78, No. 2, S. 67-9o. Vgl. ebenfalls Churchland, Patricia S.: Neurophilosophy. Toward a unified science of the mind-brain, Cambridge, Massachusetts: MIT Press 1986.

296 Mandik, Pete / Weisberg, Josh: »Type-q materialism«, 2008, Online: http://www. petemandik.com/philosophy/papers/typeq.pdf [zuletzt geprüft am 13.09.2018], S. 20.

297 Dennett, Daniel C.: »Why and How Does Consciousness Seem the Way it Seems?«, 2015, Online: http://open-mind.net/papers/why-and-how-does-consciousness-seem-the-wayit-seems [zuletzt geprüft am 24.09.2018], S. 4.

298 Die plakative Vorstellung von angeblich »naiven« gegenüber reduktionistischen Konzeptionen von Bewusstsein, nährt sich offenbar auch aus der Vorstellung eines angeblichen Konfliktes zwischen »natürlichen Psychologen« versus »professioneller Konkurrenz« (welche scheinbar die Gruppe der Reduktionisten darstellen soll). Vgl. dazu Metzinger, Thomas: Subjekt und Selbstmodell. Die Perspektivität phänomenalen Bewusstseins vor dem Hintergrund einer naturalistischen Theorie mentaler Repräsentation, Paderborn: Mentis 1999, S. 35. Stellen wir die Beweislast wenigstens in die Mitte, könnte 
beschriebenen vermeintlichen Analogien zwischen Fragen, die das hard problem angehen und solchen, die nur das easy problem betreffen. ${ }^{299}$

Ein weiterer Begriff den man als Einschüchterung gegen einen phänomenal adäquaten Zugangs zum Explanandum verstehen kann, ist der Verweis auf »Introspektion«, vor der z.B. Metzinger als einer »grossen Versuchung für alle Philosophen des Geistes ${ }^{300}$ warnt, weil erstere etwas »trügerisches « ${ }^{301}$ sei. Im Rahmen dieser Dissertation kann ich aus Platzgründen nicht weiter im Detail auf die zahlreichen, einschüchternden Wendungen, die gegen Reduktionsskeptiker/innen angeführt werden, eingehen. Weitere in der Literatur vorgefundene Formulierungen waren z.B.: »naiver Realismus «,302 »naïve conception of consciousness «, 303 »fixierte Grundannahmen «, 304 »folk psychological states « 305 oder »Introspektion « von »natürlichen Psychologen « ${ }^{306} \mathrm{Be}-$ merkenswert ist nicht zuletzt, dass im Extremfall sogar richtig feindselige Reaktionen gegenüber Reduktionsskeptiker/innen festzustellen sind. ${ }^{307}$

man eher von einem Konflikt sprechen, der darin besteht, einerseits, einer phänomenal adäquaten Bestimmung des Explanandums und andererseits der Motivation dem Desiderat der Explizierbarkeit nachzukommen.

299 Teilweise werden hard-problem-Fragen irrtümlicherweise auch mit psychologischen Fragen gleichgesetzt: Folk physics, folk biology, folk epidemiology and the like all proved to be radically mistaken. Since folk theories generally turn out to be mistaken, it seems quite improbable that folk psychology will turn out true«. Ramsey, William: »Eliminative Materialism«, in: The Stanford Encyclopedia of Philosophy, 2013, Online: http://plato. stanford.edu/archives/sum2o13/entries/materialism-eliminative/ [zuletzt geprüft am 25.09.2018], Abschnitt 3.o.

300 Metzinger, Thomas: Subjekt und Selbstmodell. Die Perspektivität phänomenalen Bewusstseins vor dem Hintergrund einer naturalistischen Theorie mentaler Repräsentation, Paderborn: Mentis 1999, S. 213.

301 A.a.O., S. 38.

302 A.a.O., S. 213.

303 Mandik, Pete / Weisberg, Josh: »Type-q materialism«, 2008, Online: http://www. petemandik.com/philosophy/papers/typeq.pdf [zuletzt geprüft am 13.09.2018], S. 20.

304 Metzinger, Thomas: Subjekt und Selbstmodell. Die Perspektivität phänomenalen Bewusstseins vor dem Hintergrund einer naturalistischen Theorie mentaler Repräsentation, Paderborn: Mentis 1999, S. 213 .

305 Ramsey, William: »Eliminative Materialism«, in: The Stanford Encyclopedia of Philosophy, 2013, Online: http://plato.stanford.edu/archives/sum2013/entries/materialismeliminative/ [zuletzt geprüft am 25.09.2018], Abschnitt 3.2.2.

306 Metzinger, Thomas: Subjekt und Selbstmodell. Die Perspektivität phänomenalen Bewusstseins vor dem Hintergrund einer naturalistischen Theorie mentaler Repräsentation, Paderborn: Mentis 1999, S. 35 .

307 Solche feindseligen Reaktionen verortet Weekes im Diskurs gegenüber zeitgenössischen (panpsychistischen) Theorien, die nicht in ein reduktionistisches Schema passen. Vgl. Weekes, Anderson: »The Mind-Body Problem and Whitehead's Non-reductive Monism«, Journal of Consciousness Studies, [2012] Vol. 19, No. 9-10, S. 51. 


\subsubsection{Hoffnung als letzter Ausweg?}

In the elementary schools of the future, that view will be taught, and the children will find a functional notion of qualia as natural as the idea that gravity makes the eraser fall to floor $[\ldots] .^{308}$

In meinen Recherchen fiel mir ein weiterer, nicht unproblematischer Umgang mit dem hard problem auf, der darin besteht, dass reduktionsoptimistische Zugänge, nach dem Abwägen aller Probleme, die immer noch auftauchen, gewissermassen ihre >letzte Zuflucht $<$ in einer Beruhigungsstrategie finden: Die schon seit mehr als sechzig Jahren ${ }^{309}$ dürftigen Fortschritte einer reduktionistischen Lösung des hard problem, scheinen nicht die Hoffnung310 nehmen zu können, dass es >irgendwann` in der Zukunft, analog zu einem easy problem gelöst werden könnte. ${ }^{311}$ So ist man schon seit mehreren Jahrzehnten stets knapp davor, das hard problem gelöst zu haben, wie Hasler, im etwas ironisch formulierten Abschnitt »Seit 50 Jahren auf der Schwelle zum Durchbruch ${ }^{312}$ treffend feststellt. Nach ihm enden denn auch viele Publikationen typischerweise mit »[...] stets auf die Zukunft verweisenden Äusserungen «.313 Die Beruhigungsstrategie beruht also im hoffnungsvollen

308 Mandik, Pete / Weisberg, Josh: »Type-q materialism«, 2008, Online: http://www. petemandik.com/philosophy/papers/typeq.pdf [zuletzt geprüft am 13.09.2018], S. 20.

309 Über die dürftigen Fortschritte im hard problem bzw. die Patt-Situation vgl. auch Walter, Sven: »Allgemeine Einleitung: Phänomenales Bewusstsein: Unlösbares Mysterium oder seriöses wissenschaftliches Problem?«, in: Qualia. Ausgewählte Beiträge, Heckmann, Heinz-Dieter / Walter, Sven [Hrsg.], Paderborn: Mentis 2001, S. 56.

310 Nach Chalmers ist die Behauptung, dass Bewusstsein physikalisch erklärt werden könne, eine unbegründete Hoffung: »[...] if a physicist or a cognitive scientist suggests that consciousness can be explained in physical terms, this is merely a hope ungrounded in current theory, and the question remains open «. Chalmers, David J.: The conscious mind. In search of a fundamental theory, New York: Oxford University Press 1996, S. xiii.

311 Die dahinter liegende Idee ist, dass die Entwicklung innovativer Technologien zu einem besseren Bild des Menschen führen werden. Vgl. dazu Demmerling, Christoph: »Schwerpunkt: Kritische Philosophie der Neurowissenschaften«, Deutsche Zeitschrift für Philosophie, [2011] Vol. 59, No. 3, S. 371.

312 Hasler, Felix: Neuromythologie. Eine Streitschrift gegen die Deutungsmacht der Hirnforschung, Bielefeld: Transcript 2012, S. 226.

313 Ebd. 
Verweis auf >zukünftigeく neurowissenschaftliche Erkenntnisse, die das hard problem $>$ sicher irgendwann ${ }^{314}$ lösen werden. ${ }^{315}$

So hofft Mandik darauf, dass es in Zukunft vielleicht »offensichtlich" werde, dass Phänomenales durch Physikalisches hervorgerufen werde, aber: $»[\ldots]$ we just may not know it yet« ${ }^{316}$ Oder Paul Churchland glaubt, dass die Phänomene des bewussten Erlebens »gewissermassen auf der Ersatzbank«317 auf das »Heranreifen der einzigen Theorie ${ }^{318}$ warten, mit der wir eine »realistische Hoffnung «319 auf ihre Erklärung hätten. An anderer Stelle hofft Churchland darauf, dass Qualia, wenn sie »letztendlich « physikalisch seien, durch »geeignete Instrumente« (»appropriate instruments«) $)^{320}$, zugänglich sein sollten. ${ }^{321}$ Im Hinblick auf diese Instrumente der Zukunft belässt er es bei

314 Vgl. dazu Levine, der konstatiert, dass der Verweis auf künftiges Wissen, auch gegen modale Argumente angewendet wird. Demnach soll wissenschaftlicher Fortschritt >irgendwann< klar machen, dass Zombies nicht vorstellbar seien. Vgl. Levin, Janet: »Functionalism«, in: The Stanford Encyclopedia of Philosophy, 2013, Online: http://plato.stanford.edu/archives/ fall2013/entries/functionalism/ [zuletzt geprüft am 28.o9.2018], Abschnitt 5.5.2.

315 Hasler stellt diesbezüglich fest, dass »[...] zur Rechtfertigung des Grabens zwischen Erklärungsanspruch und Datenlage [...] « gerne auf Zeit gespielt werde. Hasler, Felix: Neuromythologie. Eine Streitschrift gegen die Deutungsmacht der Hirnforschung, Bielefeld: Transcript 2012, S. 226.

316 Mandik, Pete / Weisberg, Josh: »Type-q materialism«, 2008, Online: http://www. petemandik.com/philosophy/papers/typeq.pdf [zuletzt geprüft am 13.09.2018], S. 7.

317 Churchland, Paul M.: »Die Wiederentdeckung des Lichtes«, in: Grundkurs Philosophie des Geistes. Band 1: Phänomenales Bewusstsein, Metzinger, Thomas [Hrsg.], Paderborn: Mentis [zuerst 1996, engl.]/2007, S. 194.

318 Ebd. Diese »einzige Theorie« besteht nach Churchland freilich in einer »reduktiven Erklärung« bzw. einer »adäquaten Theorie des Gehirns«. Ebd.

319 Im Original: »[...] the phenomena of human conscious experience have quite properly been waiting, patiently and at the sidelines, for the maturation of the only theory that has any realistic hope of providing such a reductive account, namely, an adequate theory of the brain. If and when that approach has been fully tried, and proves a failure, then, perhaps, it will be time to insist on nonphysical approaches«. Ebd. [Hervorhebung im Original].

320 Im Original: »[...] inner qualia [...] ought to be accessible as well from one or more >objective< points of view, via some appropriate instruments that scan brain activity, for example«. Churchland, Paul M.: »The Rediscovery of Light«, The Journal of Philosophy, [1996] Vol. 93, No. 5, S. 227.

321 Die das hard problem hauptsächlich betreffenden Fragen, was genau mit den Begriffen >letztendlichく bzw. >physikalischく gemeint ist, und wie Phänomenales >in oder >an diesem Physikalischen instantiiert sein könnte, lässt er leider offen. Vgl. Churchland, Paul M.: »Die Wiederentdeckung des Lichtes«, in: Grundkurs Philosophie des Geistes. Band 1: Phänomenales Bewusstsein, Metzinger, Thomas [Hrsg.], Paderborn: Mentis [zuerst 1996, engl.]/2007, S. 197. 
dem rudimentären Hinweis auf »[...] instruments that scan brain activity, for example $[\ldots] \ll .322$

Folglich kritisiert auch der Neurowissenschafter Gerhard Roth ${ }^{323}$ den »allzu grossen Optimismus « ${ }^{324}$ von Paul Churchland im Hinblick auf die Zukunft Neurowissenschaften. ${ }^{325}$ Allein schon auf der Ebene des easy problem und ungeachtet der technischen Probleme, nur schon im Hinblick auf die ungeheure Komplexität eines äusserst minimalen neuronalen Netzwerkes ${ }^{326}$ in einem einzelnen Gehirn und der diese Komplexität noch verschärfende multiplen Realisierbarkeit, ${ }^{327}$ müssten noch schier unendlich viele Graduierungen und Kombinationen von Hirnaktivitäten und damit korrelierenden Erlebniskategorien (oder noch schlimmer: einzelnen Erlebnisgraduierungen) assoziiert

322 Churchland, Paul M.: »The Rediscovery of Light«, The Journal of Philosophy, [1996] Vol. 93, No. 5 , S. 227 .

323 Gerhard Roth gilt als einschlägiger Spezialist auf den Gebieten der Neuro- und Verhaltensbiologie sowie der Neurophilosophie.

324 Roth, Gerhard: Das Gehirn und seine Wirklichkeit. Kognitive Neurobiologie und ihre philosophischen Konsequenzen, Frankfurt am Main: Suhrkamp 1994, S. 291.

325 Roth bezieht sich auf Churchlands Hoffnung, dass die Neurowissenschaften eindeutige neuronale Korrelate zu beliebigen Wahrnehmungen oder Bewusstseinszuständen bestimmen könnte. Vgl. ebd.

326 Selbst Netzwerke von nur wenigen tausend Nervenzellen, welche nur einfache Grundeinheiten kognitiver Verarbeitung darstellen, sind von einer ausserordentlichen Komplexität. Vgl. a.a.O., S. 277. Nur schon diese geringe Anzahl von etwa tausend Nervenzellen umfassen eine bis zehn Millionen Synapsen. Vgl. a.a.O., S. 276. Insgesamt umfasst das menschliche Gehirn jedoch selbst nach den neueren, etwas bescheideneren Schätzungen, ca. 86'оoo'ooo'ooo Neuronen (und die Anzahl der Glia-Zellen, die ebenfalls funktionelle Neurotransmitterrezeptoren besitzen, und deshalb mindestens potenziell die Funktion besitzen, neuronale Aktivität zu detektieren, übertrifft die der Neuronen um das Zehnfache)! Vgl. Bear, Mark F. / Connors, Barry W. / Paradiso, Michael A.: Neuroscience. Exploring the brain, Philadelphia: Wolters Kluwer 2012, S. 28, 50 und 52.

Zudem weist ein einziges Neuron ca. 29'ooo Synapsen auf und der Dendritenbaum eines einzelnen Neurons kann mit ca. 100'oooo bis 200'ooo Nervenfasern in Verbindung treten (Dendriten sind für die Aufnahme neuronaler Erregung und für dessen Fortleitung zum Zellkörper zuständig). Vgl. dazu Jäncke, Lutz: Lehrbuch Kognitive Neurowissenschaften, Bern: Huber 2013, S. 67-68. Zur Funktion von Dendriten vgl. auch Roth, Gerhard: Das Gehirn und seine Wirklichkeit. Kognitive Neurobiologie und ihre philosophischen Konsequenzen, Frankfurt am Main: Suhrkamp 1994, S. 40.

327 Multiple Realisierbarkeit bedeutet, dass es für jeden mentalen Zustand multiple Weisen der Instantiierungen gibt. Einfach gesagt, bedeutet das, dass mentale Zustände durch verschiedene hardware physikalisch realisierbar sind. Zu jedem mentalen Prozess gibt es also mehr als nur ein mögliches neurophysiologisches Korrelat. Die Komplexität würde deshalb noch grösser, wenn wir, wie es empirisch ja auch nötig wäre, nicht nur ein einzelnes Gehirn in jenem erhofften `Zukunftsscanner< untersuchen müssten. Vgl. dazu a.a.O., S. 277. Vgl. ebenfalls Schröder, Jürgen: »Qualia und Physikalismus«, Journal for General Philosophy of Science, [1997] Vol. 28, No. 1, S. 179. 
werden. Berücksichtigt man die Komplexität des ganzen Gehirns, wird die Lage für jene Zukunftsmaschine noch schwieriger: nur schon im Balken (Corpus callosum) gibt es z.B. einen >Informationsverkehr bis sechstausend Milliarden Ereignissen pro Sekunde. ${ }^{328}$

Zudem geht Churchlands besagter Verweis auf allfällige Instrumente der Zukunft nicht auf die grundsätzliche Problematik ein, dass derartige Hoffnungen relativ leicht unterminiert werden können, weil sie nicht isoliert vom hard problem betrachtet werden dürfen: Selbst wenn es >irgendwann< einmal, trotz aller Komplexitätsprobleme, ein solches »geeignetes Instrument « ${ }^{329}$ der Zukunft geben würde, so ist damit noch nicht das schwerwiegende Problem gelöst, wie damit die subjektive Perspektive durch eine »objektive «330 Perspektive »zugänglich « (»accessible«) ${ }^{331}$ gemacht werden soll. Demgemäss kritisiert Elitzur, dass auch ein, vielleicht in der Zukunft mögliches Wissen, wie jedes einzelne Neuron ganz genau bei einem Erlebnis feuert, im Hinblick auf das hard problem nicht nur nichts nützt, sondern das Problem dadurch sogar noch grösser wird. ${ }^{332}$ Dies trifft für Elitzur schon bei relativ einfachen Erfahrungsweisen, wie z.B. dem Sehen einer Rose zu.

Qualia elude not only communication, but observation and experiment as well. Suppose that, with sufficiently advanced technology, you obtain the fullest realtime description of what goes on in my brain - every neuron, synapse and neurotransmitter molecule - when I see a red rose. [...] Paradoxically, the problem now becomes worse: You know better than I do what goes on in my brain when I perceive red, and yet, that doesn't bring you any closer to my quale of red. ${ }^{333}$

Infolgedessen wäre auch mit der Erfüllung von Churchlands Hoffnung auf jenes »geeignete Instrument « ${ }^{334}$ der Zukunft der Kern des hard problem nach

328 Genauer: allein das Corpus callosum bzw. der Balken, enthält ungefähr sechshundert Millionen Nervenfasern. Eine dieser Faser sendet pro Sekunde zwischen ca. 40 bis 1000 Signale. Dies addiert sich zu einem >Informationsverkehr von zweihundert bis sechstausend Milliarden Ereignissen pro Sekunde alleine im Balken. Vgl. Baars, Bernard J.: Das Schauspiel des Denkens. Neurowissenschaftliche Erkundungen, Stuttgart: Klett-Cotta 1998, S. 97.

329 Churchland, Paul M.: »The Rediscovery of Light«, The Journal of Philosophy, [1996] Vol. 93, No. 5 , S. 227 .

330 Ebd.

331 Ebd.

332 Vgl. Elitzur, Avshalom C.: »Consciousness makes a difference: A reluctant dualist's confession«, 2009, Online: http://philpapers.org/archive/ELICMA.pdf [zuletzt geprüft am 13.09.2018], S. 5 .

333 A.a.O., S. 5 [Hervorhebung im Original].

334 Churchland, Paul M.: »The Rediscovery of Light«, The Journal of Philosophy, [1996] Vol. 93, No. 5 , S. 227 . 
wie vor nicht überwunden. Könnte tatsächlich nachgewiesen werden, dass das hard problem irgendwann in der Zukunft analog zu einem easy problem gelöst werden kann, so wäre das hard problem im Prinzip jetzt schon gelöst. Doch wenn dies nicht der Fall ist, und selbst wenn >irgendwann < in der Zukunft die Daten so exakt und vollständig wären, dass wir alle Nuancen subjektiven Erlebens wie z.B. auch Emotionen ${ }^{335}$ messen könnten, müssten Beschreibungen wie $>38 \%$ Erstaunen, $32 \%$ Vergnügen, $12 \%$ Ärger etc<. mit Emotions-Begriffen verknüpft werden, die als solche nur aus dem je eigenen subjektiven Erleben überhaupt eine Referenz besitzen. ${ }^{336}$ Ein weiterer damit zusammenhängender Grund, der die Hoffnungen auf eine >zukünftige neurowissenschaftliche Lösung des hard problem schwer untergräbt, liegt auch darin, dass die Neurowissenschaften den »[...] deutungsoffenen humanities strukturell näher [...] $\ll^{337}$ stehen als beispielsweise der anorganischen Chemie.

Ein zentraler, aber weitgehend übersehener Faktor scheint zu sein, dass sich SCAN [soziale, kognitive und affektive Neurowissenschaften] in einem wichtigen Punkt gerade nicht von geisteswissenschaftlichen Verfahren und Diskurstypen unterscheidet: Neurowissenschaftliches Forschen, wenn es den menschlichen Geist oder menschliche Fähigkeiten und Merkmale zum Gegenstand hat, ist auf einem hermeneutischen Untergrund errichtet, der aber so gut wie nie mitreflektiert wird. ${ }^{338}$

335 Mehr über die Phänomenalität von emotionalem Erleben vgl. Abschnitte 8.2.3 und 8.2.4.

336 Mit anderen Worten: falls jenes von Churchland erhoffte »geeignete Instrument« irgendwann einmal so weit sein wird, dass es auf eine irgendeine Weise feststellen könnte, dass Chalmers, während er visuellen Fokus auf Churchlands Aufsatz The Mystery of David Chalmers richtet, ein emotionales Erlebnis hat, welches sich exakt in den ersten 20 Sekunden der Messperiode als eine Mischung von 38\% Erstaunen, 32\% Vergnügen 20\% Gespanntheit und 10\% Empörung anfühlt, sich dann aber in der Zusammensetzung in Richtung Ärger verschiebt etc., so hätte Churchland einen epistemischen Zugang zum Erleben von Chalmers nur aufgrund eigener und mit diesen >gemessenen< Emotionen ähnlichen phänomenalen Erfahrungen aus seiner Erinnerung. Das heisst, Churchland müsste eigene Emotions-Erinnerungen mit den Daten jenes `Scanners` verknüpfen, damit die Messergebnisse $>38 \%$ Erstaunen, 32\% Vergnügen $20 \%$ Gespanntheit und $10 \%$ Empörungく, wie von ihm behauptet, als Qualia durch eine >objektiveく Perspektive »zugänglich « (»accessible«) gemacht werden sollen. Ebd. Das heisst, Churchland müsste die vom Hirnscan gemessenen Emotionen mit eigenen phänomenalen Erfahrungen, die er z.B. mit den Begriffen 〉Gespanntheit< oder `Empörungく assoziiert, anreichern, damit er sich vorstellen könnte, was denn Chalmers während des Hirnscans erlebt. Es bliebe für Churchland aber immer nur eine Vorstellung. Damit wäre, selbst mit dieser technisch noch kaum vorstellbaren Errungenschaft, kein >objektiver« epistemischer Zugang zum Erleben gewonnen.

337 Slaby, Jan: »Perspektiven einer kritischen Philosophie der Neurowissenschaften«, Deutsche Zeitschrift für Philosophie, [2011] Vol. 59, No. 3, S. 377 [Hervorhebung im Original].

338 Ebd. [Anmerkung in Klammern von E.E.]. 


\subsection{6 »Sachfremde Motive« hat immer der Antipode}

Das berechtigte Bedenken von Bieri, dass phänomenaler Realismus ${ }^{339}$ und skeptische Haltungen gegenüber reduktionistischen Ansätzen vielleicht auf »sachfremden Motiven «340 gründen könnten, sollte man im Zusammenhang mit den weiter unten beschriebenen Ängsten vor dem Dualismus, auch andernorts anbringen: Genauso berechtigt ist die Frage, ob es nicht ebenso viele »sachfremde Motive ${ }^{341}$ auf der Seite von Reduktionsoptimisten und Qualia-Gegnern gibt, die durch eine kritische Analyse des im Diskurs verwendeten Qualia-Begriffs ins Licht rücken könnten. Es stellt sich nämlich die >ketzerische< Frage, ob die Bedeutungseinengung und Bedeutungsverschiebungen von Qualia vielleicht Strategien sein könnten, die im $\mathrm{Zu}-$ sammenhang mit dem Wunsch stehen, Phänomenales zu eliminieren oder zu marginalisieren, um ebenso das mit ihm verbundene harte Problem der Explizierbarkeit eliminieren zu können. Denn wenn es Bewusstsein und Erleben gibt, so glaubt man, besteht die Gefahr in einen Dualismus abzugleiten. Liegt also hinter den zahlreichen Bedeutungseinengungen und Bedeutungsverschiebungen vom Explanandum des hard problem vielleicht die Motivation, Eigenschaftsdualismus, Panpsychismus oder, noch gefürchteter, einen ontologischen Dualismus um jeden Preis zu vermeiden?

Meines Erachtens waren die verschiedenen Formen des Behaviorismus und Funktionalismus niemals durch eine unabhängige Untersuchung der Fakten motiviert, sonder durch die Befürchtung, wir wären dem Dualismus und einem anscheinend unlösbaren Körper/Geist-Problem ausgeliefert, solange sich kein Weg fände, geistige Phänomene [...] zu eliminieren. ${ }^{342}$

339 Bieri spricht diesbezüglich nicht vom phänomenalen Realismus, sondern von der »Introspektion«, die dem »wissenschaftlichen Realismus« zufolge nur eine »wahre Theorie« sein könne, wenn der Kontext der »Introspektion« eine Theorie sei, die das Ergebnis empirischer Forschung sei, die »[...] nicht an unsere momentanen Meinungen gebunden ist - wie tief verwurzelt diese Meinungen auch sein mögen«. Bieri, Peter [Hrsg.]: Analytische Philosophie des Geistes, Weinheim: Beltz 2007, S. 24. Ebenfalls seien wir immer wieder auf Begriffsanalysen angewiesen, um uns darüber klar zu werden, was unsere »[...] gewöhnlichen mentalistischen Beschreibungen von Personen implizieren«. A.a.O., S. 25 .

340 A.a.O., S. 24.

341 Ebd.

342 Searle, John R. / [Übers.: Gavagai, Harvey]: Intentionalität. Eine Abhandlung zur Philosophie des Geistes, Frankfurt am Main: Suhrkamp [zuerst 1989, engl.]/1991, S. 11 [Hervorhebung E. E.]. 
Nicht selten scheint jedoch die Aufgabe, »sachfremde $«^{343}$ Motive aufzudecken, implizit reduktionistisch-naturalistischen Ansätzen zugewiesen zu werden. ${ }^{344}$ So stellt Bieri fest, die Aufgabe der Philosophie des Geistes sei eine diagnostische, um solche Motive aufzudecken. Denn die Philosophiegeschichte zeige, dass hinter Theorien des Geistes oft »Motive « ${ }^{345}$ stehen, die mit einer unbefangenen Analyse des Mentalen nichts zu tun hätten. Nach Bieri bestehen diese Motive oft in folgender Fragestellung: »Was müssen wir über mentale Phänomene sagen, damit wir an bestimmten Prinzipien, die wir nicht aufgeben wollen, festhalten können? «346 Diese prinzipiengeleitete Frage könnte freilich sowohl von reduktionskritischen als auch von reduktionsaffinen Vertretern der Philosophie des Geistes stammen, denn es lässt sich mit Recht behaupten, dass in reduktionistisch-physikalistischen oder eliminativistischen Positionen ebenfalls Motive vorhanden sind, bestimmte Grundannahmen »nicht aufgeben« zu wollen. Die Grundannahmen dieser Positionen sind vermutlich darum so schwer aufzugeben, weil sie von der oben beschriebenen Hoffnung genährt werden, dass das hard problem sicher >irgendwann< (im Sinne eines reduktiven Materialismus) gelöst werde. So könnte die Motivation, subjektives Erleben auf Hirnprozesse reduzieren zu wollen, auch darin bestehen, »[...] die physikalische Welt von allem Erlebten und Lebendigen zu reinigen $[. ..] \ll, 347$ weil die mit Subjektivität verbundene $»$ Leiblichkeit $«^{348}$ vielleicht die ${ }^{4}$ Achillesferse des Neuroreduktionismus $«^{349}$ ist. Das Vermeiden der schwierigen Frage über das Verhältnis zwischen dem Physischen und dem Mentalen ist deshalb, nach Nagel, eines der wichtigsten Motive des Reduktionismus. ${ }^{350}$ Ähnlich argumentiert Searle, der im Hinblick auf ein äusserst unbequemes Explanandum eine »hidden agenda « ${ }^{351}$ in der Philosophie des Geistes verortet, die darin besteht, das Mentale irgendwie loszuwerden:

343 Bieri, Peter [Hrsg.]: Analytische Philosophie des Geistes, Weinheim: Beltz 2007, S. 24.

344 Vgl. a.a.O., S. 9-24.

345 A.a.O., S. 24.

346 Ebd.

347 Fuchs, Thomas: »Hirnwelt oder Lebenswelt? Zur Kritik des Neurokonstruktivismus«, Deutsche Zeitschrift für Philosophie, [2011] Vol. 59, No. 3, S. 356.

348 Ebd.

349 Ebd.

35 O Vgl. Nagel, Thomas: Geist und Kosmos. Warum die materialistische neodarwinistische Konzeption der Natur so gut wie sicher falsch ist, Berlin: Suhrkamp 2013, S. 81.

351 Searle, John R.: The mystery of consciousness, New York: New York Review Books 1997, S. 136 . 
The history of the philosophy of mind over the past one hundred years has been in large part an attempt to get rid of the mental by showing that no mental phenomena exist over and above physical phenomena. [...] The materialist philosopher purports to offer an analysis of the mental, but his or her hidden agenda is to get rid of the mental. ${ }^{352}$

Demgemäss vermutet auch Chalmers bei Einwänden ${ }^{353}$ gegen modale und epistemische Argumente, die sich auf unsere kognitive Beschränktheit (»cognitive impairment «) ${ }^{354}$ berufen, nur um den Materialismus zu retten, eine Motivation, den Materialismus »um jeden Preis« zu retten (»to hang onto materialism at all costs «). ${ }^{355}$

Trotz oder gerade wegen dieser Schwierigkeiten sieht Dennett solche Motive jedoch nur bei den Qualia-Befürwortern: »[...] many are unwilling to take my radical challenge seriously largely because they want so much for qualia to be acknowledged «. ${ }^{356}$ Qualia sind für ihn gewissermassen die letzte Bastion, der letzte Verteidigungsgraben der »Innerlichkeit« des Mentalen (»Inwardness of our minds «). ${ }^{357}$ Eine Bastion, die Dennett zufolge, im Falle ihrer NichtPlausibilität, von der Wissenschaft eingenommen werde: »otherwise their last bastion of specialness will be stormed by science «. ${ }^{358}$ Auch Papineau vermutet sachfremde Motive offenbar ausschliesslich bei den philosophischen Antipoden. So glaubt er tatsächlich, die Erklärungslücke auf die Frage reduzieren zu können, warum Reduktionsskeptiker/innen eine »Abwehrhaltung « ${ }^{359}$ hätten, seine Identitätstheorie zu akzeptieren.

Ein Indiz über allfällige Motive von Qualia-Gegnern findet sich bei Beckermann. Er spricht explizit von »Strategien [...] auf die eine oder andere

$35^{2}$ Ebd. [Hervorhebung E. E.].

353 Er bezieht sich dabei auf Ad-hoc-Argumente gegen modale Argumente, die zwar zugeben würden, dass Zombies vorstellbar sind, aber dennoch davon ausgingen, dass sie auch metaphysisch nicht möglich seien, was wiederum mit unserer kognitiven Beschränktheit (»cognitive impairment «) begründet wird. Chalmers, David J.: The conscious mind. In search of a fundamental theory, New York: Oxford University Press 1996, S. 139.

354 Ebd.

355 A.a.O., S. 140.

356 Dennett, Daniel C.: »Quining Qualia«, in: Philosophy of mind. Classical and contemporary readings, Chalmers, David J. [Hrsg.], New York: Oxford University Press [zuerst 1988]/2002, S. 229 [Hervorhebung E. E.].

357 Ebd.

$35^{8}$ Ebd.

359 Papineau, David: »Mind the Gap«, Philosophical Perspectives, [1998] Vol. 12: Language, Mind, and Ontology, S. 384. 
Weise mit den anti-physikalistischen Intuitionen fertig zu werden «. ${ }^{360}$ Die Motivation hinter diesen Strategien könnte also darin bestehen, dass QualiaGegner irrtümlich davon ausgehen, dass die Anerkennung phänomenaler Explananda >nur mit »anti-physikalistischen Intuitionen $\ll^{361} \mathrm{zu}$ tun hätten und dass mit der Leugnung der Erste-Person-Perspektive, die mit ihr verbundenen explanatorischen Probleme ebenfalls aus der Welt geschafft würden. Ein zentrales Motiv, dem Phänomenalen auszuweichen oder ihm die >Existenz $\prec$ abzusprechen, bestünde demnach darin, die mit diesem Explanandum verbundenen und von Qualia-Gegnern auch zugegebenen »erheblichen theoretischen Probleme $«^{362} \mathrm{zu}$ vermeiden. Robinson spricht demgemäss von einer »Notwendigkeit«, die aus bewusstem Erleben folgenden $»$ kontraintuitiven« Epiphänomene zu »vermeiden«, weshalb wir »verpflichtet« seien, eine »plausible« Form des »materialistischen Monismus« (»a plausible form of materialist monism « $)^{363} \mathrm{zu}$ entwerfen.

Laut Bieri, verdanken wir der analytischen Philosophie des Geistes und ihrer logischen Geographie der mentalistischen Sprache, begriffliche Entdeckungen, hinter die keine Theorie des Geistes zurückgehen könne. ${ }^{364}$ Die leitende Frage, die wir uns im Hinblick auf das hard problem stellen müssen, sollte, wenn wir das Explanandum nicht scheuen, dennoch wie folgt sein: was können wir, aufgrund aller momentan verfügbaren Evidenzen über mentale Phänomene sagen? ${ }^{365} \mathrm{Zu}$ diesen Evidenzen gehört aber nicht zuletzt der Umstand, dass wir mentale Phänomene erleben. Und dieser Umstand, dass wir erleben, ist nicht nur einfach ein >bloss subjektiver Umstand, sondern etwas, das von uns intersubjektiv ${ }^{366}$ bestätigt werden kann; deshalb spricht Nagel diesbezüglich

36o Beckermann, Ansgar: Analytische Einführung in die Philosophie des Geistes, Berlin: W. de Gruyter 2008, S. 445 .

361 Ebd.

362 A.a.O., S. 454 .

363 Robinson, Howard: »Dualism«, in: The Stanford Encyclopedia of Philosophy, 2013, Online: http://plato.stanford.edu/archives/fall2o13/entries/dualism/ [zuletzt geprüft am 25.09.2018], Abschnitt 1.2.

364 Vgl. Bieri, Peter [Hrsg.]: Analytische Philosophie des Geistes, Weinheim: Beltz 2007, S. 13.

365 Vgl. dazu auch a.a.O., S. 24.

366 Auch wenn wir keinen Zugriff auf das Erleben anderer haben, so teilen wir intersubjektiv die Erfahrung, dass wir erleben. Natürlich kann jemand auch glauben, dass alle anderen, ausser er oder sie selbst, Zombies sind und dass diese Zombies von ihrem >Erleben< nur berichten, obwohl sie kein Erleben haben. Mit einem derart starken Skeptizismus kann man natürlich auch die Existenz aller Materie, die Existenz der Existenz bzw. des gesamten Universums anzweifeln. Selbstverständlich können wir letztlich weder beweisen, dass wir bewusst sind, noch dass eine Welt und ein Universum existiert. 
von »Tatsachen «. ${ }^{367}$ Dabei ist es eigentlich zweitrangig, ob wir das, was wir $>$ Erleben` nennen, nun als erlebte >Täuschung oder als >phänomenale Tatsache< interpretieren. Das damit zusammenhängende Explanandum phänomenaler Bewusstseinszustände gehört infolgedessen ebenfalls zu den Evidenzen mentaler Phänomene. ${ }^{368}$ Dies erzwingt es, in der leitenden Fragestellung einer Theorie des Geistes, die mit dem hard problem auch zu tun haben soll, ohne Wenn und Aber (und ohne Zuweisungen von »sachfremden Motiven ${ }^{369}$ nur beim philosophischen Antipoden $)^{370}$ das Desiderat phänomenaler Adäquatheit zu berücksichtigen.

367 »Facts about what it is like to be an X [...]《. Nagel, Thomas: »What is it like to be a bat?«, Philosophical Review, [1974] Vol. 83, No. 4, S. 437. Vgl. dazu auch a.a.O., S. 441.

368 Natürlich kann man dem hard problem mit der Strategie ausweichen, dass man einfach von der Prämisse ausgeht, dass es das Erleben mentaler Phänomene gar nicht >gibt $<$ bzw. dass es keine phänomenale Erfahrung >gibt<, was immer das auch heissen mag. Dann gäbe es auch kein zu erklärendes Explanandum des hard problem. Es mag an dieser Stelle schwer zu verstehen sein, dass Personen, die nicht bewusstlos sind, überhaupt daran zweifeln können, dass sie Bewusstsein haben. Vgl. dazu auch Searle, John R. / [Übers.: Gavagai, Harvey]: Die Wiederentdeckung des Geistes, München: Artemis-Verlag \& Winkler [zuerst 1992, engl.]/1993, S. 126. Im Kontext der oben erwähnten Motive wird diese Prämisse, wenn man sie in strategischer Hinsicht betrachtet, etwas verständlicher. Inwiefern eine solche Strategie heuristisch und nicht bloss rhetorisch gewinnbringend ist, soll hier dahingestellt bleiben. In den Abschnitten 7.1, 9.1 und 10.2.2 wird auf dieses Problem noch etwas näher eingegangen.

369 Bieri, Peter [Hrsg.]: Analytische Philosophie des Geistes, Weinheim: Beltz 2007, S. 24. Bieri lässt es jedoch mehr oder weniger offen, auf welcher Seite diese Motive auftreten. Allerdings stellt er fest, dass die »Introspektion« nur zutreffen könne, wenn sie mit empirischer Forschung übereinstimme, was (je nach Verständnis von >Introspektion<) dem objektivistischen Zugang zum Phänomenalen implizit einen gewissen Vorrang einzuräumen könnte. Vgl. dazu ebd.

370 Wenn schon, dann lassen sich Motive sowohl auf Seiten der Reduktionskritiker/Innen als auch der Reduktionsoptimisten/Innen finden. 